Pure and Applied Mathematics Quarterly

Volume 6, Number 1

(Special Issue: In honor of

John Tate, Part 2 of 2$)$

$83-172,2010$

\title{
On Leading Terms and Values of Equivariant Motivic $L$-functions
}

\author{
David Burns
}

To John Tate on the occasion of his eightieth birthday

\begin{abstract}
The 'Equivariant Tamagawa Number Conjecture' formulated by Flach and the present author is a natural refinement of the seminal Tamagawa Number Conjecture that was originally formulated by Bloch and Kato and then subsequently extended and refined by Kato and by Fontaine and Perrin-Riou. We explain how the additional information implicit in this refinement entails a variety of explicit predictions concerning both the leading terms and values of equivariant motivic $L$-functions and the module structure of integral motivic cohomology groups. We also discuss several concrete applications of this approach in the setting of Artin and Hasse-Weil $L$-functions.
\end{abstract}

Keywords: motivic $L$-functions, leading terms, Euler characteristics, module structures, Fitting ideals, congruences.

\section{INTRODUCTION}

Let $K / k$ be a finite Galois extension of number fields and set $G:=\operatorname{Gal}(K / k)$. For any motive $M$ (in the sense of $\S 4.1$ ) that is defined over $k$ we regard the motive $M_{K}:=h^{0}(\operatorname{Spec} K) \otimes_{h^{0}(\operatorname{Spec} k)} M$ as defined over $k$ and endowed with a natural left action of the group ring $\mathbb{Q}[G]$ (via the first factor). We write

Received January 6, 2007.

1991 Mathematics Subject Classification: Primary 11S40; Secondary 11R29 20C05. 
$\zeta(\mathbb{C}[G])$ for the centre of $\mathbb{C}[G]$ and $L^{*}\left(M_{K}\right)$ for the leading term in the Taylor expansion at $s=0$ of the natural $\zeta(\mathbb{C}[G])$-valued $L$-function of $M_{K}$. Then the 'Equivariant Tamagawa Number Conjecture' that is formulated by Flach and the present author in $\left[23\right.$, Conj. 4] predicts for the pair $\left(M_{K}, \mathbb{Z}[G]\right)$ an equality of the form

$$
\delta\left(L^{*}\left(M_{K}\right)\right)=\chi\left(M_{K}\right) .
$$

Here $\delta$ is a canonical homomorphism from the unit group of $\zeta(\mathbb{R}[G])$ to the relative algebraic $K$-group of the ring extension $\mathbb{Z}[G] \subseteq \mathbb{R}[G]$ and $\chi\left(M_{K}\right)$ is an Euler characteristic that is defined by using virtual objects (in the sense of Deligne) arising from the various motivic cohomology groups, realisations, comparison isomorphisms and regulators associated to both $M_{K}$ and its Kummer dual. This equality refines the seminal 'Tamagawa Number Conjecture' that was originally formulated by Bloch and Kato in [9] and then extended and refined by both Kato [64, 65] and Fontaine and Perrin-Riou [47].

Flach's article [45] contains a survey of evidence in support of the Equivariant Tamagawa Number Conjecture. Our aim in the present article however is to help explain the interest of the conjecture by describing some general methods for converting the rather involved conjectural equality (1) into predictions that are much more explicit in nature. As such, this article is also in part a survey of known results but in addition contains a variety of results and explicit predictions that are, as far as we are aware, completely new. These predictions concern Euler characteristic formulas, restrictions on the structures of natural arithmetic modules, formulas relating components of the leading term $L^{*}\left(M_{K}\right)$ to the Fitting ideals of such modules and congruences between components of $L^{*}\left(M_{K}\right)$ that involve natural algebraic height pairings. Most of the explicit relations we derive from the conjectural equality (1) will depend on the fact that it is formulated using structures in $M_{K}$ that are projective with respect to $\mathbb{Z}[G]$ rather than a maximal order in $\mathbb{Q}[G]$ (so, in particular, most of the relations that we describe for Tate motives do not follow from the Tamagawa Number Conjecture for Artin motives that is described, for example, in [61, §1.2]) and hence do indeed reflect consequences of (1) that do not follow from the original 'non-equivariant' conjecture of Bloch and Kato.

Our basic approach to deriving explicit consequences of (1) is to abstract existing results and techniques from special cases to the general setting of 'augmented trivialized extensions' that we introduce in $\S 3$. There are several ways in which 
this approach seems to be useful and interesting. Firstly, it shows that a wide variety of well known, comparatively explicit and hitherto seemingly unrelated conjectures (including, for example, conjectures formulated by Stark [90, 94], Gross [56], Chinburg [29, 30, 31], Lichtenbaum [71], Chinburg, Kolster, Pappas and Snaith [33], Brumer, Coates and Sinnott [36], Gross [55], Popescu [82], Rubin [86], Tate [95] and Mazur and Tate [73]) are manifestations of a single underlying principle. Secondly, it gives rise to concrete refinements of several of these much studied conjectures and also to the formulation of new conjectures of a rather concrete nature. These new conjectures include, under certain hypotheses, Euler characteristic formulas for Tate motives (Remark 6.2.4) and elliptic curves (Remark 6.2.5), restrictions on the module structure of algebraic $K$-groups (Proposition 7.2.5) and Mordell-Weil groups (Remark 7.1.5 and Proposition 7.2.6(i) and (iii)), an interplay between orders of Tate-Shafarevic groups and orders of vanishing of motivic $L$-functions (Proposition 7.2.6(ii)) and a 'strong main conjecture' of the kind that Mazur and Tate ask for in [73, Remark after Conj. 3] (see Remark 8.2.5). Other explicit consequences of the approach developed here that will be considered in detail elsewhere include the predicted existence in natural non-abelian contexts of 'special elements' in algebraic $K$ groups and Mordell-Weil groups that are related to the first derivatives of Artin and Hasse-Weil $L$-functions (Remark 5.1.5) and predicted congruences between higher derivatives of Hasse-Weil $L$-functions (Remark 9.5.4). Further, modulo certain standard expectations, our approach applies to motives far more general than Tate motives and those obtained from elliptic curves (cf. §4.4) and in each such case our methods should allow the formulation of similarly explicit predictions. The third respect in which our approach is useful is that any evidence for the explicit consequences of (1) that we derive would also constitute evidence for the conjectures formulated by Fukaya and Kato in [51] and also for the main conjecture of non-commutative Iwasawa theory formulated by Coates, Fukaya, Kato, Sujatha and Venjakob in [35]. Indeed, whilst it is clear that (1) is a consequence of the 'non-commutative Tamagawa number conjecture' formulated in [51], in [28] Venjakob and the present author have shown that the relevant special case of (1) also follows from (a natural refinement of) the central conjecture of Coates et al in [35]. 
In a little more detail the main contents of this article is as follows. In $\S 2$ we review necessary algebraic preliminaries. In $\S 3$ we introduce a notion of 'augmented trivialized extension' (or 'a.t.e.' for short) and in $\S 4$ we prove that in many natural cases the conjectural equality (1) is equivalent to the vanishing of the 'Euler characteristic' of a suitable a.t.e. In $\S 5$ we state our main algebraic results. These results describe certain explicit consequences of the vanishing of the Euler characteristic of an arbitrary a.t.e. and are proved in $\S 6-\S 9$ using a suitable abstraction of arguments that have already been used in special cases by previous authors. In these sections we also discuss several arithmetic applications and formulate a variety of new and explicit conjectures (see Remark 5.1.2 for more details regarding these conjectures). In an attempt to stress the principles which underlie our general approach we choose to postpone the proofs of several technical results to the end of the article. Such results include a natural 'Hasse Principle' for morphisms in derived categories (in $\S 10$ ), the answer to a question posed by Chinburg et al in [33] (in §11) and a detailed study of the conjectural equality (1) for motives arising from elliptic curves (in $\S 12$ ).

An early version of this article was first circulated in 2004 and the article was completed in 2007 when the author held a Leverhulme Research Fellowship. It is a pleasure to thank Adebisi Agboola, Werner Bley, Matthias Flach, Dick Gross, Kazuya Kato, Masato Kurihara and Jan Nekovář for stimulating discussions. I am also very grateful to Griff Elder for providing me with a proof of Proposition 7.2.1, to Daniel Macias Castillo for corrections to an earlier version of $\S 9.4$ and to the referee for several helpful remarks. John Tate has provided much inspiration and generous encouragement to me and it is certainly a very great pleasure to dedicate this article to him on the occasion of his eightieth birthday.

\section{Algebraic Preliminaries}

In this section we quickly review some necessary algebraic preliminaries. All modules are to be considered, unless explicitly stated otherwise, as left modules.

2.1. Basic categories. Let $R$ be any (associative unital) ring. We write $\operatorname{PMod}(R)$, resp. $\operatorname{PMod}(R)^{\bullet}$, for the category of finitely generated projective $R$-modules, resp. of bounded complexes of such modules. We also write $D(R)$ for the derived category of complexes of $R$-modules and $D^{\text {perf }}(R)$ for the full triangulated subcategory of $D(R)$ consisting of those complexes which are 'perfect' (that is, 
isomorphic in $D(R)$ to an object of $\left.\operatorname{PMod}(R)^{\bullet}\right)$. We say that an $R$-module $N$ is 'perfect' if the complex $N[0]$ belongs to $D^{\text {perf }}(R)$.

2.2. Virtual objects. In [39] Deligne proves that there exists a 'universal determinant functor' $[\cdot]_{R}$ from the subcategory of $\operatorname{PMod}(R)$ where morphisms are restricted to isomorphisms to the category $V(R)$ of 'virtual objects' associated to $\operatorname{PMod}(R)$. We recall that $V(R)$ has a canonical bifunctor $\left(L_{1}, L_{2}\right) \mapsto L_{1} L_{2}$ and 'unit object' $\mathbf{1}_{R}$ which induce on $V(R)$ the structure of Picard category. In particular, for each object $L$ of $V(R)$ there is an 'inverse object' $L^{-1}$ in $V(R)$ that is unique up to unique isomorphism and an isomorphism $L L^{-1} \stackrel{\sim}{\longrightarrow} \mathbf{1}_{R}$ in $V(R)$. The groups $\pi_{0}(V(R))$ and $\pi_{1}(V(R))$ are naturally isomorphic to the Grothendieck group $K_{0}(R)$ of $\operatorname{PMod}(R)$ and to the Whitehead group $K_{1}(R)$ of $R$. We recall that $K_{1}(R)$ can be described explicitly in terms of generators of the form $(M, \mu)$ where $M$ is any finitely generated projective $R$-module and $\mu$ is any element of $\operatorname{Aut}_{R}(M)$ (cf. [37, (38.28)]).

If $R$ is commutative, then $V(R)$ identifies with the category of graded invertible $R$-modules (and isomorphisms of such) and $[\cdot]_{R}$ with the determinant functor described by Knudsen and Mumford in [67] (see $\S 8$ for more details).

For further details concerning the general (non-commutative) case and the extension of $[\cdot]_{R}$ from $\operatorname{PMod}(R)$ to $D^{\text {perf }}(R)$ see $[23, \S 2]$.

2.3. Relative algebraic $K$-theory. Let $\Lambda$ be a Dedekind domain of characteristic zero and write $F$ for its quotient field. We fix a finite dimensional semisimple $F$-algebra $A$ and a $\Lambda$-order $\mathfrak{A}$ in $A$ (so $\mathfrak{A}$ is a subring of $A$ that is finitely generated as a $\Lambda$-module and generates $A$ as an $F$-module). For any extension field $E$ of $F$ and any $A$-module $N$ we set $N_{E}:=E \otimes_{F} N$, regarded as an $A_{E}$-module in the natural way.

Following [23] we define $V\left(\mathfrak{A}, A_{E}\right)$ to be the fibre product category

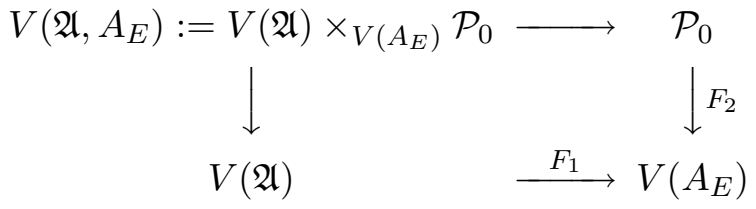

where $\mathcal{P}_{0}$ is the Picard category with unique object $\mathbf{1}_{\mathcal{P}_{0}}$ and $\operatorname{Aut}_{\mathcal{P}_{0}}\left(\mathbf{1}_{\mathcal{P}_{0}}\right)=0, F_{2}$ is the (monoidal) functor sending $\mathbf{1}_{\mathcal{P}_{0}}$ to $\mathbf{1}_{A_{E}}$ and $F_{1}\left([L]_{\mathfrak{A}}\right)=\left[E \otimes_{\Lambda} L\right]_{A_{E}}$ for each 
object $L$ of $\operatorname{PMod}(\mathfrak{A})$. Then $V\left(\mathfrak{A}, A_{E}\right)$ is a Picard category and (from [23, Prop. 2.5]) there is a natural isomorphism of (abelian) groups

$$
\iota_{\mathfrak{A}, A_{E}}: \pi_{0}\left(V\left(\mathfrak{A}, A_{E}\right)\right) \cong K_{0}\left(\mathfrak{A}, A_{E}\right) .
$$

The 'relative algebraic $K$-group' $K_{0}\left(\mathfrak{A}, A_{E}\right)$ used here is defined as a quotient of the free abelian group on symbols of the form $\left(Q_{1}, Q_{2} ; \phi\right)$ where $Q_{1}$ and $Q_{2}$ belong to $\operatorname{PMod}(\mathfrak{A})$ and $\phi$ is an isomorphism of $A_{E}$-modules $E \otimes_{\Lambda} Q_{1} \cong E \otimes_{\Lambda} Q_{2}$ (see [92, p. 215] for an explicit description of the relations used to define this quotient). For each object $X$ of $D^{\text {perf }}(\mathfrak{A})$ and morphism $\lambda:\left[E \otimes_{\Lambda} X\right] \rightarrow \mathbf{1}_{A_{E}}$ in $V\left(A_{E}\right)$ we set

$$
[X, \lambda]:=\iota_{\mathfrak{A}, A_{E}}((X, \lambda)) \in K_{0}\left(\mathfrak{A}, A_{E}\right) .
$$

(In Lemma 3.2.1 we give a description of such elements in terms of the explicit generators of $\left.K_{0}\left(\mathfrak{A}, A_{E}\right)\right)$.

There is a natural commutative diagram of exact sequences of the form

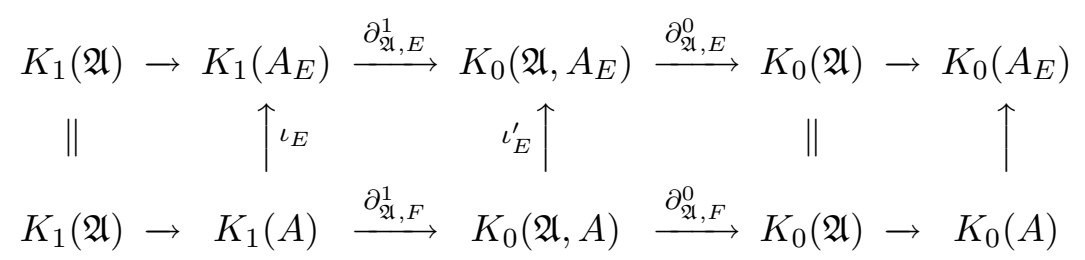

(cf. [92, Th. 15.5]). Here the homomorphisms $\iota_{E}$ and $\iota_{E}^{\prime}$ are induced by the scalar extensions $\phi \mapsto E \otimes_{F} \phi$ and $\left(Q_{1}, Q_{2} ; \phi\right) \mapsto\left(Q_{1}, Q_{2} ; E \otimes_{F} \phi\right)$ and are both injective (indeed, we often regard these maps as inclusions). The homomorphism $\partial_{\mathfrak{A}, E}^{1}$ sends the class of an automorphism $\phi$ of $A_{E}^{n}$ to $\left(\mathfrak{A}^{n}, \mathfrak{A}^{n} ; \phi\right)$ and $\partial_{\mathfrak{A}, E}^{0}$ sends $\left(Q_{1}, Q_{2} ; \phi\right)$ to $\left(Q_{1}\right)-\left(Q_{2}\right)$ where $(Q)$ denotes the class in $K_{0}(\mathfrak{A})$ of each $Q$ in $\operatorname{PMod}(\mathfrak{A})$.

2.4. Reduced norms. We write $\zeta(R)$ and $R^{\times}$for the centre and unit group of a ring $R$. If $R$ is a finite dimensional semisimple algebra over either a global field or the completion of such a field, then one can compute in $K_{1}(R)$ by using the injective reduced norm homomorphism $\operatorname{Nrd}_{R}: K_{1}(R) \rightarrow \zeta(R)^{\times}$(as discussed in $[37, \S 45 \mathrm{~A}])$.

If $G$ is a finite group, then the square of every element of $\zeta(\mathbb{R}[G])^{\times}$belongs to $\operatorname{im}\left(\operatorname{Nrd}_{\mathbb{R}[G]}\right)$ but the inclusion $\operatorname{im}\left(\operatorname{Nrd}_{\mathbb{R}[G]}\right) \subseteq \zeta(\mathbb{R}[G])^{\times}$can be strict (see (13)). However, for each finitely generated subring $\Lambda$ of $\mathbb{Q}$ there always exists a canonical 
'extended boundary homomorphism'

$$
\delta_{\Lambda}: \zeta(\mathbb{R}[G])^{\times} \rightarrow K_{0}(\Lambda[G], \mathbb{R}[G])
$$

which satisfies

$$
\delta_{\Lambda} \circ \operatorname{Nrd}_{\mathbb{R}[G]}=\partial_{\Lambda[G], \mathbb{R}}^{1}
$$

on $K_{1}(\mathbb{R}[G])$ and is such that $\zeta(\mathbb{Q}[G])^{\times}$is equal to the full pre-image of $K_{0}(\Lambda[G]$, $\mathbb{Q}[G])$ under $\delta_{\Lambda}\left(\right.$ cf. $[23, \S 4.2]$ ). If $\Lambda=\mathbb{Z}$, then we often abbreviate $\delta_{\Lambda}$ to $\delta$.

The Wedderburn decomposition of $\mathbb{C}[G]$ induces a canonical decomposition

$$
\zeta(\mathbb{C}[G])=\prod_{\chi \in \operatorname{Irr}(G)} \mathbb{C}
$$

where $\operatorname{Irr}(G)$ is the set of irreducible finite-dimensional $\mathbb{C}$-valued characters of $G$. We write $x=\left(x_{\chi}\right)_{\chi}$ for the corresponding decomposition of each element $x$ of $\zeta(\mathbb{C}[G])$.

2.5. Involutions. Let $G$ be a finite group and $\Lambda$ a finitely generated subring of $\mathbb{Q}$. If $Q$ belongs to $\operatorname{PMod}(\Lambda[G])$, then the linear dual $Q^{*}:=\operatorname{Hom}_{\Lambda}(Q, \Lambda)$ also belongs to $\operatorname{PMod}(\Lambda[G])$ when endowed with the natural contragredient $G$-action. Hence, one obtains involutions $\psi^{*}$ of both $K_{0}(\Lambda[G], \mathbb{R}[G])$ and $K_{0}(\Lambda[G])$ by setting $\psi^{*}\left(\left(Q_{1}, Q_{2} ; \phi\right)\right):=\left(Q_{1}^{*}, Q_{2}^{*} ; \operatorname{Hom}_{\mathbb{R}}(\phi, \mathbb{R})^{-1}\right)$ and $\psi^{*}(Q):=\left(Q^{*}\right)$ respectively. With these definitions it is clear that $\partial_{\Lambda[G], \mathbb{R}}^{0} \circ \psi^{*}=\psi^{*} \circ \partial_{\Lambda[G], \mathbb{R}}^{0}$.

We write $x \mapsto x^{\#}$ for the involution of $\zeta(\mathbb{C}[G])$ that is induced by the $\mathbb{C}$-linear anti-involution of $\mathbb{C}[G]$ sending each element of $G$ to its inverse. Then $\left(x^{\#}\right)_{\chi}=x_{\bar{\chi}}$ for each $\chi \in \operatorname{Irr}(G)$, where $\bar{\chi}$ is the contragredient of $\chi$. In addition, [23, Lem. 16] implies that for each $x$ in $\zeta(\mathbb{R}[G])^{\times}$one has

$$
\psi^{*}\left(\delta_{\Lambda}(x)\right)=-\delta_{\Lambda}\left(x^{\#}\right) \in K_{0}(\Lambda[G], \mathbb{R}[G]) .
$$

2.6. Cohomological-triviality. Let $G$ be a finite group and $\Lambda$ a Dedekind domain. We say that a $\Lambda[G]$-module $M$ is 'cohomologically-trivial', or occasionally just 'c-t' for short, if for each subgroup $J$ of $G$ and each integer $i$ the Tate cohomology group $\hat{H}^{i}(J, M)$ vanishes. This condition is equivalent to $M$ having finite projective dimension as a $\Lambda[G]$-module and is automatically satisfied in, for example, each of the following cases:-

- $M$ is a projective $\Lambda[G]$-module;

- $M=\Lambda[G] \otimes_{\Lambda} N$ for any $\Lambda$-module $N$; 
- $M$ is a finite module of order prime to the order of $G$;

- The order of $G$ is invertible in $\Lambda$.

If $M$ is finitely generated, then it is cohomologically-trivial if and only if it is perfect (in the sense of $\S 2.1$ ). Further, if $M$ is any such module, and $P^{\bullet}$ is any object of $\operatorname{PMod}(\Lambda[G])^{\bullet}$ that is isomorphic in $D(\Lambda[G])$ to $M[0]$, then the Euler characteristic $(M):=\sum_{i \in \mathbb{Z}}(-1)^{i}\left(P^{i}\right)$ is an element of $K_{0}(\Lambda[G])$ that is independent of the precise choice of $P^{\bullet}$.

2.7. Further notation. Unadorned tensor products are to be considered as taken in the category of $\mathbb{Z}$-modules. If $\Lambda$ is clear from context, then for any finite group $G$ and $\Lambda[G]$-module $N$ we set $N^{*}:=\operatorname{Hom}_{\Lambda}(N, \Lambda)$ and $N^{\vee}:=$ $\operatorname{Hom}_{\Lambda}(N, \mathbb{Q} / \Lambda)$, each endowed unless explicitly stated otherwise with contragredient $G$-action. We also let $N_{\text {tor }}$ denote the $\Lambda$-torsion submodule of $N$ and set $N_{\mathrm{tf}}:=N / N_{\text {tor }}$. We also write $N^{G}$, resp. $N_{G}$, for the maximal sub-module, resp. quotient module, upon which $G$ acts trivially. If $E$ is a number field, resp. discrete valuation ring, then $\mathcal{O}_{E}$ will denote its ring of algebraic integers, resp. valuation ring. For each prime $p$ we write $\mathbb{Z}_{(p)}$ and $\mathbb{Z}_{p}$ for the $p$-localisation of $\mathbb{Z}$ and the ring of $p$-adic integers respectively. The cardinality of a finite set $X$ is denoted $|X|$.

\section{2-EXTENSIONS}

In this section we introduce the algebraic construction that plays an essential role in our approach to describing explicit consequences of the conjectural equality (1).

3.1. The definition. As in $\S 2.3$ we fix a Dedekind domain $\Lambda$ of characteristic zero and quotient field $F$, an extension field $E$ of $F$, a finite dimensional semisimple $F$-algebra $A$ and a $\Lambda$-order $\mathfrak{A}$ in $A$.

Given finitely generated $\mathfrak{A}$-modules $H^{0}$ and $H^{1}$ we shall say that an element $\epsilon$ of $\operatorname{Ext}_{\mathfrak{A}}^{2}\left(H^{1}, H^{0}\right)$ is 'perfect' if it can be represented (as a Yoneda extension) by an exact sequence

$$
0 \rightarrow H^{0} \rightarrow M^{0} \rightarrow M^{1} \rightarrow H^{1} \rightarrow 0
$$

in which the $\mathfrak{A}$-modules $M^{0}$ and $M^{1}$ are both perfect. 
Perfect extension classes arise naturally in arithmetic in the following way. If $C^{\bullet}$ is an object of $D^{\text {perf }}(\mathfrak{A})$ that is acyclic outside degrees 0 and 1 and $\iota_{i}$ : $H^{i}\left(C^{\bullet}\right) \cong H^{i}$ is an isomorphism of $\mathfrak{A}$-modules for both $i \in\{0,1\}$, then the tautological exact sequence

$$
0 \rightarrow H^{0}\left(C^{\bullet}\right) \rightarrow C^{0} / B^{0}\left(C^{\bullet}\right) \rightarrow Z^{1}\left(C^{\bullet}\right) \rightarrow H^{1}\left(C^{\bullet}\right) \rightarrow 0
$$

(where $B^{0}\left(C^{\bullet}\right)$ and $Z^{1}\left(C^{\bullet}\right)$ are the boundaries and cocyles of $C^{\bullet}$ in degrees 0 and 1) combines with $\iota_{0}$ and $\iota_{1}$ to specify an element $\epsilon$ of $\operatorname{Ext}_{\mathfrak{A}}^{2}\left(H^{1}, H^{0}\right)$. It is straightforward to check that $\epsilon$ is perfect and also unchanged if one replaces $\left(C^{\bullet}, \iota_{0}, \iota_{1}\right)$ by any similar triple $\left(C^{\bullet \bullet}, \iota_{0}^{\prime}, \iota_{1}^{\prime}\right)$ for which there is an isomorphism $\iota: C^{\bullet} \cong C^{\bullet \bullet}$ in $D^{\text {perf }}(\mathfrak{A})$ with $\iota_{i}^{\prime} \circ H^{i}(\iota)=\iota_{i}$ for both $i \in\{0,1\}$. We shall say that any such triple is a 'representative' of $\epsilon$.

If now $\epsilon \in \operatorname{Ext}_{\mathfrak{A}}^{2}\left(H^{1}, H^{0}\right)$ is perfect and $\psi: E \otimes_{\Lambda} H^{0} \cong E \otimes_{\Lambda} H^{1}$ is an isomorphism of $A_{E}$-modules, then we set

$$
[\epsilon, \psi]:=\iota_{\mathfrak{A}, A_{E}}\left(\left(\left[C^{\bullet}\right]_{\mathfrak{A}} ; \psi_{\text {Triv }}\right)\right) \in K_{0}\left(\mathfrak{A}, A_{E}\right)
$$

where $\left(C^{\bullet}, \iota_{0}, \iota_{1}\right)$ is any representative of $\epsilon$ and $\psi_{\text {Triv }}$ is the composite

$$
\begin{aligned}
{\left[E \otimes_{\Lambda} C^{\bullet}\right]_{A_{E}} \rightarrow \prod_{i \in \mathbb{Z}}\left[H^{i}\left(E \otimes_{\Lambda} C^{\bullet}\right)\right]_{A_{E}}^{(-1)^{i}} } & \rightarrow\left[E \otimes_{\Lambda} H^{0}\right]_{A_{E}}\left[E \otimes_{\Lambda} H^{1}\right]_{A_{E}}^{-1} \\
& \rightarrow\left[E \otimes_{\Lambda} H^{1}\right]_{A_{E}}\left[E \otimes_{\Lambda} H^{1}\right]_{A_{E}}^{-1} \rightarrow \mathbf{1}_{A_{E}}
\end{aligned}
$$

where the first arrow is the canonical morphism that is induced by the semisimplicity of the $E$-algebra $A_{E}$ (for an explicit description of this morphism see [12, Rem. 3.2]), the second arrow is the morphism induced by the given isomorphisms $\iota_{0}$ and $\iota_{1}$ and the fact that $H^{i}\left(E \otimes_{\Lambda} C^{\bullet}\right)=0$ if $i \notin\{0,1\}$ and the third arrow is the morphism induced by $\psi$. (It is easy to check that this definition of $[\epsilon, \psi]$ is independent of the precise choice of $\left(C^{\bullet}, \iota_{0}, \iota_{1}\right)$.)

Definition 3.1.1. An augmented $E$-trivialized extension of $\mathfrak{A}$-modules is a triple $\tau=\left(\epsilon_{\tau}, \lambda_{\tau}, \mathcal{L}_{\tau}^{*}\right)$ comprising a perfect 2-extension $\epsilon_{\tau} \in \operatorname{Ext}_{\mathfrak{A}}^{2}\left(H_{\tau}^{1}, H_{\tau}^{0}\right)$ of (finitely generated) $\mathfrak{A}$-modules, an isomorphism $\lambda_{\tau}: E \otimes_{\Lambda} H_{\tau}^{0} \cong E \otimes_{\Lambda} H_{\tau}^{1}$ of $A_{E}$-modules and an element $\mathcal{L}_{\tau}^{*}$ of $\zeta\left(A_{E}\right)^{\times}$. If $F=\mathbb{Q}$, then by an a.t.e. of $\mathfrak{A}$-modules we shall mean an augmented $\mathbb{R}$-trivialized extension of $\mathfrak{A}$-modules. We define the Euler characteristic $\chi(\tau)$ of an augmented E-trivialized extension of $\mathfrak{A}$-modules $\tau$ by setting

$$
\chi(\tau):=\left[\epsilon_{\tau}, \lambda_{\tau}\right]-\delta_{\Lambda}\left(\mathcal{L}_{\tau}^{*}\right) \in K_{0}\left(\mathfrak{A}, A_{E}\right) .
$$


The arithmetical examples of augmented trivialised extensions that are to be discussed in $\S 4$ show that it is reasonable to regard $\left[\epsilon_{\tau}, \lambda_{\tau}\right]$ and $\delta_{\Lambda}\left(\mathcal{L}_{\tau}^{*}\right)$ as the 'algebraic' and 'analytic' components of $\chi(\tau)$.

3.2. An explicit interpretation. The definition of the element $[\epsilon, \psi]$ in $(6)$ in terms of virtual objects is important both for comparison to the general constructions of Flach and the present author in [23] and also for the purposes of routine calculations using the Euler characteristic formalism developed by Breuning and the present author in [12] and by Breuning in [11]. However, in arithmetic applications it is also often advantageous to use the following description of the Euler characteristic of an a.t.e. in terms of explicit generating elements of $K_{0}(\Lambda[G], F[G])$. We note in particular that claim (ii) of the following result shows that the vanishing of the Euler characteristic of an a.t.e. $\tau$ implies that the element $\mathcal{L}_{\tau}^{*}$ satisfies a certain type of congruence relation.

For any $G$-module $N$ and natural number $d$ we write $N^{\langle d\rangle}$ for the direct sum of $d$ copies of $N$.

Lemma 3.2.1. Let $G$ be a finite group and $\Lambda$ a finitely generated subring of $\mathbb{Q}$. Let $\tau$ be an a.t.e. of $\Lambda[G]$-modules for which $H_{\tau}^{0}$ is torsion-free.

(i) There exist finitely generated projective $\Lambda[G]$-modules $P^{0}$ and $P^{1}$ lying in an extension

$$
0 \rightarrow H_{\tau}^{0} \rightarrow P^{0} \stackrel{d}{\rightarrow} P^{1} \stackrel{\pi}{\rightarrow} H_{\tau}^{1} \rightarrow 0
$$

of class $\epsilon_{\tau}$ and for which

$$
\left[\epsilon_{\tau}, \lambda_{\tau}\right]=\left(P^{0}, P^{1} ; \iota\right)
$$

Here $\iota$ denotes the composite isomorphism

$$
\begin{aligned}
\mathbb{R} \otimes_{\Lambda} P^{0} \cong\left(\mathbb{R} \otimes_{\Lambda} H_{\tau}^{0}\right) \oplus\left(\mathbb{R} \otimes_{\Lambda} \operatorname{im}(d)\right) & \\
& \cong\left(\mathbb{R} \otimes_{\Lambda} H_{\tau}^{1}\right) \oplus\left(\mathbb{R} \otimes_{\Lambda} \operatorname{im}(d)\right) \cong \mathbb{R} \otimes_{\Lambda} P^{1}
\end{aligned}
$$

where the first and third isomorphisms are induced by a choice of $\mathbb{R}[G]$ equivariant sections to the surjective homomorphisms $\mathbb{R} \otimes_{\Lambda} P^{0} \rightarrow$ $\mathbb{R} \otimes_{\Lambda} \operatorname{im}(d)$ and $\mathbb{R} \otimes_{\Lambda} P^{1} \rightarrow \mathbb{R} \otimes_{\Lambda} H_{\tau}^{1}$ that are induced by $d$ and $\pi$ respectively, and the second isomorphism is $\left(-\lambda_{\tau}\right) \oplus \mathrm{id}$. 
(ii) Let $d_{\tau} \in\{1,2\}$ be the least strictly positive integer such that $\left(\mathcal{L}_{\tau}^{*}\right)^{d_{\tau}}$ belongs to the image of $\operatorname{Nrd}_{\mathbb{R}[G]}$. If $d_{\tau} \cdot \chi(\tau)=0$, then in claim (i) one can choose $P^{0}$ and $P^{1}$ so that $P^{0\left\langle d_{\tau}\right\rangle}=P^{1\left\langle d_{\tau}\right\rangle}$ is a finitely generated free $\Lambda[G]$-module and in $\zeta(\mathbb{R}[G])^{\times}$one has

$$
\left(\mathcal{L}_{\tau}^{*}\right)^{d_{\tau}} \equiv \operatorname{Nrd}_{\mathbb{R}[G]}\left(\left(\mathbb{R} \otimes_{\Lambda} P^{0\left\langle d_{\tau}\right\rangle}, \iota^{\left\langle d_{\tau}\right\rangle}\right)\right)
$$

modulo the image under $\operatorname{Nrd}_{\mathbb{R}[G]}$ of $\operatorname{im}\left(K_{1}(\Lambda[G]) \rightarrow K_{1}(\mathbb{R}[G])\right)$, where ${ }_{\iota}\left\langle d_{\tau}\right\rangle$ is the automorphism of $\mathbb{R} \otimes_{\Lambda} P^{0\left\langle d_{\tau}\right\rangle}$ that is induced by $\iota$, resp. by $\iota \oplus \iota$, if $d_{\tau}=1$, resp. $d_{\tau}=2$.

Proof. We set $\epsilon:=\epsilon_{\tau}, \lambda:=\lambda_{\tau}$ and $H^{i}:=H_{\tau}^{i}$ for $i=0,1$.

If one computes $\operatorname{Ext}_{\Lambda[G]}^{2}\left(H^{1}, H^{0}\right)$ by means of a projective resolution of $H^{1}$, then it is clear that one can choose a representative (7) of $\epsilon$ in which $P^{1}$ is a finitely generated free $\Lambda[G]$-module (of arbitrarily large rank). Then, having fixed such a module $P^{1}$, the fact that $\epsilon$ is perfect implies that there exists a quasi-isomorphism of complexes of $\Lambda[G]$-modules of the form $\alpha: Q^{\bullet} \rightarrow P^{\bullet}$ where $Q^{\bullet}$ belongs to $\operatorname{PMod}(\Lambda[G])^{\bullet}$ and $P^{\bullet}$ denotes the complex $P^{0} \stackrel{d}{\rightarrow} P^{1}$, where $P^{0}$ is placed in degree 0 . From the acyclicity of the mapping cone of $\alpha$ one then deduces that $P^{0}$ is both finitely generated and cohomologically-trivial as a $G$-module. But, since both $P^{1}$ and (by assumption) $H^{0}$ are torsion-free, the exactness of (7) implies that $P^{0}$ is also torsion-free. Thus, by $[2$, Th. 8$], P^{0}$ is a finitely generated projective $\Lambda[G]$ module, as claimed. In addition, the claimed equality $[\epsilon, \lambda]=\left(P^{0}, P^{1} ; \iota\right)$ follows from results of $[12]$. Indeed, the element $[\epsilon, \lambda]=\iota_{\Lambda[G], \mathbb{R}[G]}\left(\left(\left[P^{\bullet}\right]_{\Lambda[G]} ; \lambda_{\text {Triv }}\right)\right)$ is equal to the element $\chi_{\Lambda[G], \mathbb{R}[G]}\left(P^{\bullet}, \lambda\right)$ defined in $[12$, Def. 5.5$]$, whilst $\left(P^{0}, P^{1} ; \iota\right)$ is equal to the element $-\chi^{\text {old }}\left(P^{\bullet},(-\lambda)^{-1}\right)$ that is defined at the beginning of $[12, \S 6]$. The equality $[\epsilon, \lambda]=\left(P^{0}, P^{1} ; \iota\right)$ thus follows upon combining the results of $[12$, Th. 6.2 and Rem. 6.4] with the fact that $\chi^{\text {old }}\left(P^{\bullet},(-\lambda)^{-1}\right)$ differs from $\chi^{\text {old }}\left(P^{\bullet}, \lambda^{-1}\right)$ by the image under $\partial_{\Lambda[G], \mathbb{R}}^{1}$ of the element $\left(\mathbb{R} \otimes_{\Lambda} H^{0},-\mathrm{id}\right)$ of $K_{1}(\mathbb{R}[G])$. This completes the proof of claim (i).

To prove claim (ii) we assume that $d_{\tau} \cdot \chi(\tau)=0$. Then in $K_{0}(\Lambda[G])$ one has $0=\partial_{\Lambda[G], \mathbb{R}}^{0}(0)=\partial_{\Lambda[G], \mathbb{R}}^{0}\left(d_{\tau} \cdot \chi(\tau)\right)=\partial_{\Lambda[G], \mathbb{R}}^{0}\left(d_{\tau} \cdot[\epsilon, \lambda]\right)-\partial_{\Lambda[G], \mathbb{R}}^{0}\left(\delta\left(\left(\mathcal{L}_{\tau}^{*}\right)^{d_{\tau}}\right)\right)=$ $\partial_{\Lambda[G], \mathbb{R}}^{0}\left(d_{\tau} \cdot[\epsilon, \lambda]\right)=\left(P^{0\left\langle d_{\tau}\right\rangle}\right)-\left(P^{1\left\langle d_{\tau}\right\rangle}\right)$, where the penultimate equality follows from (3), the exactness of the upper row of (2) and the containment $\left(\mathcal{L}_{\tau}^{*}\right)^{d_{\tau}} \in$ $\operatorname{im}\left(\operatorname{Nrd}_{\mathbb{R}[G]}\right)$ and the last equality from the description of $[\epsilon, \lambda]$ that is given in claim (i). 
Using the same argument as in the proof of claim (i), we now choose $P^{1}$ to be a finitely generated free $\Lambda[G]$-module of rank at least two. From the existence of $\lambda_{\tau}$ we may deduce that the $\mathbb{Q}[G]$-modules $\mathbb{Q} \otimes_{\Lambda} H_{\tau}^{0}$ and $\mathbb{Q} \otimes_{\Lambda} H_{\tau}^{1}$ are isomorphic (cf. [37, Exer. 6, p. 139]). In addition, for each prime $p$ the algebra $\mathbb{Q}_{p}[G]$ is semisimple and so we may choose splittings of the exact sequence that results from applying $\mathbb{Q}_{p} \otimes_{\Lambda}-$ to $(7)$ : in this way, one finds that $\mathbb{Q}_{p} \otimes_{\Lambda} P^{0}$ is isomorphic to the free $\mathbb{Q}_{p}[G]$-module $\mathbb{Q}_{p} \otimes_{\Lambda} P^{1}$. Thus, since $P^{0}$ is projective, Swan's Theorem [37, Th. 32.1] implies that $\mathbb{Z}_{p} \otimes_{\Lambda} P^{0}$ is a free $\mathbb{Z}_{p}[G]$-module for each prime $p \in \operatorname{Spec}(\Lambda)$. From Jacobinski's Cancellation Theorem we may therefore deduce that $P^{0\left\langle d_{\tau}\right\rangle}$ is isomorphic to $P^{1\left\langle d_{\tau}\right\rangle}$ as a $\Lambda[G]$-module: indeed, the equality $\left(P^{0\left\langle d_{\tau}\right\rangle}\right)=\left(P^{1\left\langle d_{\tau}\right\rangle}\right)$ in $K_{0}(\Lambda[G])$ implies that $P^{0\left\langle d_{\tau}\right\rangle}$ is stably isomorphic to $P^{1\left\langle d_{\tau}\right\rangle}$ and then, since the $\Lambda[G]$-rank of $P^{0\left\langle d_{\tau}\right\rangle}$ is at least two, this implies that $P^{0\left\langle d_{\tau}\right\rangle}$ is isomorphic to $P^{1\left\langle d_{\tau}\right\rangle}$ by $[37,(49.3),(51.30)]$. Hence we can assume that $P^{0\left\langle d_{\tau}\right\rangle}=P^{1\left\langle d_{\tau}\right\rangle}$ is a finitely generated free $\Lambda[G]$-module, as asserted in claim (ii). Further, in this case the element $d_{\tau} \cdot\left(P^{0}, P^{1} ; \iota\right)$ is equal to $\partial_{\Lambda[G], \mathbb{R}}^{1}\left(\left(\mathbb{R} \otimes_{\Lambda} P^{0\left\langle d_{\tau}\right\rangle}, \iota^{\left\langle d_{\tau}\right\rangle}\right)\right.$ and so the exactness of the upper row of (2) implies that if $d_{\tau} \cdot \chi(\tau)=0$, then $\left(\mathbb{R} \otimes_{\Lambda} P^{0\left\langle d_{\tau}\right\rangle},{ }_{\iota}\left\langle d_{\tau}\right\rangle\right)$ differs from the inverse image of $\left(\mathcal{L}_{\tau}^{*}\right)^{d_{\tau}}$ under the (injective) $\operatorname{map} \operatorname{Nrd}_{\mathbb{R}[G]}$ by an element of $\operatorname{im}\left(K_{1}(\Lambda[G]) \rightarrow K_{1}(\mathbb{R}[G])\right)$. It is then clear that the latter condition is equivalent to the stated congruence.

Remark 3.2.2. The element $\left(P^{0}, P^{1} ; \iota\right)$

Since the isomorphism $\iota$ defined in Lemma 3.2.1(i) involves the homomorphism $d$ the element $\left(P^{0}, P^{1} ; \iota\right)$ depends upon the complex $P^{0} \stackrel{d}{\rightarrow} P^{1}$ (and the identification of its cohomology with $H_{\tau}^{0}$ and $H_{\tau}^{1}$ via the sequence $\left.(7)\right)$ rather than just on the modules $P^{0}$ and $P^{1}$.

\section{Arithmetic examples}

In this section we show that the algebraic construction of Definition 3.1.1 arises naturally in interesting arithmetic contexts. At a first reading, the reader may prefer to look only at the general discussion of $\S 4.1$ and at the statements of Propositions 4.2.2, 4.2.5, 4.2.6 and 4.3.1 (and perhaps also the discussion of $\S 4.4$ ) and then pass straight on to $\S 5$.

We now fix a number field $k$ and an algebraic closure $k^{c}$ of $k$ and set $G_{k}:=$ $\operatorname{Gal}\left(k^{c} / k\right)$. We also fix a finite Galois extension $K$ of $k$ inside $k^{c}$ and set $G:=$ $\operatorname{Gal}(K / k)$. 
4.1. Motives. To formulate the Equivariant Tamagawa Number Conjecture it is sufficient to regard motives over $k$ as specified by their realisations and motivic cohomology groups and the usual maps between these groups (that is, by a 'motivic structure' in the sense of Fontaine and Perrin-Riou [47]). In this subsection we review a few details of this approach, referring any interested reader to [23, $\S 3]$ for further details.

If $M=h^{n}(X)(r)$ for a smooth projective variety $X$ over $k$ together with an integer $r$ and a non-negative integer $n$, then its realisations are as follows. A filtered $k$-space $H_{\mathrm{dR}}(M):=H_{\mathrm{dR}}^{n}\left(X_{/ k}\right)$ with the natural decreasing filtration $F^{i} H_{\mathrm{dR}}(M):=F^{i} H_{\mathrm{dR}}^{n}\left(X_{/ k}\right)$ shifted by $r$; for each prime $\ell$ a compatible system of $\ell$-adic representations $H_{\ell}(M):=H_{\text {ét }}^{n}\left(k^{c} \times_{k} X, \mathbb{Q}_{\ell}(r)\right)$ of $G_{k}$; for each embedding $\sigma: k \rightarrow \mathbb{C}$ a $\mathbb{Q}$-Hodge structure $H_{\sigma}(M):=H^{n}\left(\sigma X(\mathbb{C}),(2 \pi i)^{r} \mathbb{Q}\right)$ over either $\mathbb{R}$ or $\mathbb{C}$ according to whether $\sigma$ factors though the inclusion $\mathbb{R} \subset \mathbb{C}$ or not.

For each motive $M$ over $k$ there is a natural left action of $\mathbb{Q}[G]$ on the motive $M_{K}:=h^{0}(\operatorname{Spec} K) \otimes_{h^{0}(\operatorname{Spec} k)} M$, where the tensor is the tensor product in the category of motives (so, for example, if $M=h^{n}(X)(r)$ as above, then $M_{K}=$ $\left.h^{n}\left(K \times_{k} X\right)(r)\right)$. For the realisations one has $H_{\mathrm{dR}}\left(M_{K}\right)=K \otimes_{k} H_{\mathrm{dR}}(M)$ where $G$ acts in the natural way on $K ; H_{\ell}\left(M_{K}\right)=\left(\prod_{\tau} \mathbb{Q}_{\ell}\right) \otimes_{\mathbb{Q}_{\ell}} H_{\ell}(M)$ where $\tau$ runs over the set of $k$-embeddings $K \rightarrow k^{c}, G$ acts only on the first factor (via precomposition with the embeddings) and $G_{k}$ acts diagonally on both factors (on the first factor via post-composition with the embeddings $) ; H_{\sigma}\left(M_{K}\right)=\left(\prod_{\sigma^{\prime}} \mathbb{Q}\right) \otimes_{\mathbb{Q}}$ $H_{\sigma}(M)$ where $\sigma^{\prime}$ runs over all embeddings $K \rightarrow \mathbb{C}$ which restrict to $\sigma$ on $k$ and $G$ acts in the natural way on the set of such embeddings $\sigma^{\prime}$.

In now $\Lambda$ is any finitely generated subring of $\mathbb{Q}$ and $\mathfrak{A}$ any $\Lambda$-order in $\mathbb{Q}[G]$ that contains $\Lambda[G]$, then the approach of [23] leads, in general modulo the validity of certain standard conjectures, to the definition of a canonical element $T \Omega\left(M_{K}, \mathfrak{A}\right)$ of $K_{0}(\mathfrak{A}, \mathbb{R}[G])$. The conjecture ${ }^{\prime} \operatorname{ETNC}\left(M_{K}, \mathfrak{A}\right)$ ' formulated in [23, Conj. 4(iv)] for the pair $\left(M_{K}, \mathfrak{A}\right)$ simply asserts that the element $T \Omega\left(M_{K}, \mathfrak{A}\right)$ vanishes. From the functorial properties of the elements $T \Omega\left(M_{K}, \mathfrak{A}\right)$ it therefore follows that if $\Lambda^{\prime}$ is any finitely generated subring of $\mathbb{Q}$ that contains $\Lambda$ and $\mathfrak{B}$ any $\Lambda^{\prime}$-order in $\mathbb{Q}[G]$ that contains $\mathfrak{A}$, then $\operatorname{ETNC}\left(M_{K}, \mathfrak{A}\right)$ implies $\operatorname{ETNC}\left(M_{K}, \mathfrak{B}\right)$ (and in particular therefore that $\operatorname{ETNC}\left(M_{K}, \mathbb{Z}[G]\right)$ implies $\operatorname{ETNC}\left(M_{K}, \mathfrak{A}\right)$ for all such orders $\left.\mathfrak{A}\right)$. 
4.2. Tate motives. For each integer $r$ we always abbreviate the 'Tate motive' $h^{0}(\operatorname{Spec} K)(r)$ to $\mathbb{Q}(r)_{K}$. We recall that for any $\Lambda$-order $\mathfrak{A}$ in $\mathbb{Q}[G]$ with $\Lambda[G] \subseteq \mathfrak{A}$ the definition in $[25, \S 3.1]$ of $T \Omega\left(\mathbb{Q}(r)_{K}, \mathfrak{A}\right)$ is unconditional.

\section{Remark 4.2.1. Supporting evidence}

Before proceeding, we recall that there is by now a fair amount of evidence in support of $\operatorname{ETNC}\left(\mathbb{Q}(r)_{K}, \mathbb{Z}[G]\right)$. In particular, this conjecture is known to be valid in each of the following cases.

- $r$ is any integer, $K$ is any abelian extension of $\mathbb{Q}$ and $k$ is any subfield of $K$ : this is proved by Flach and the present author in [25, Cor. 1.2].

- $r=0, k$ is an imaginary quadratic field of class number one and $G$ is an abelian group of order divisible only by primes which split completely in $k / \mathbb{Q}$ : this is proved by Bley in $[6$, Th. 4.2$]$.

- $r=0$ and $r=1, k=\mathbb{Q}$ and $K$ belongs to a certain infinite family of fields for which $G$ is isomorphic to the Quaternion group of order eight: these cases follow from results of Flach and the present author in [24, Th. 4.1] and [25, (1) and Cor. 1.5] and rely heavily on results of Chinburg in [32]. - $r=0, k=\mathbb{Q}$ and $K$ is a particular field for which $G$ is isomorphic to the alternating group of order twelve: this is the main result of Navilarekallu in $[76$, Th. 3].

In addition to the above cases in which $\operatorname{ETNC}\left(\mathbb{Q}(r)_{K}, \mathbb{Z}[G]\right)$ has been verified completely, Nickel [79] has recently described evidence in favour of the conjecture in the case that $r=0$ and $K$ is a tamely ramified CM Galois extension of a totally real field $k$.

We now fix a finite set $S$ of places of $k$ that contains the set $S_{\infty}$ of archimedean places and also all that ramify in $K / k$. For each character $\psi \in \operatorname{Irr}(G)$ we write $L_{S}(\psi, s)$ for the $S$-truncated Artin $L$-function as defined (using the arithmetic Frobenius!) in [94, Chap. 0, $\S 4$. For each integer $r$ we set $L_{K / k, S}^{*}(r):=$ $\left(L_{S}^{*}(\psi, r)\right)_{\psi} \in \zeta(\mathbb{C}[G])^{\times}$where $L_{S}^{*}(\psi, r)$ is the leading term in the Laurent expansion of $L_{S}(\psi, s)$ at $s=r$. Then, since $L_{S}^{*}(\bar{\psi}, r)$ is equal to the complex conjugate of $L_{S}^{*}(\psi, r)$ for each character $\psi$, the element $L_{K / k, S}^{*}(r)$ actually belongs to $\zeta(\mathbb{R}[G])^{\times}$. 
4.2.1. $\mathbb{Q}(0)_{K}$. We write $Y_{K, S}$ for the free abelian group on the set $S(K)$ of places of $K$ which lie above those in $S$ and $X_{K, S}$ for the kernel of the homomorphism $Y_{K, S} \rightarrow \mathbb{Z}$ that sends each element of $S(K)$ to 1 . We write $\mathcal{O}_{K, S}$ for the subring of $K$ consisting of those elements that are integral at all places outside $S(K)$ and $\mathcal{O}_{K, S}^{\times}$for the group of units of $\mathcal{O}_{K, S}$.

Proposition 4.2.2. There exists an a.t.e. of $\mathbb{Z}[G]$-modules $\tau_{0}$ with all of the following properties:-

- $H_{\tau_{0}}^{0}=\mathcal{O}_{K, S}^{\times},\left(H_{\tau_{0}}^{1}\right)_{\text {tor }}=\operatorname{Pic}\left(\mathcal{O}_{K, S}\right)$ and $\left(H_{\tau_{0}}^{1}\right)_{\mathrm{tf}}=X_{K, S}$;

- $\lambda_{\tau_{0}}$ is induced by -1 times the Dirichlet regulator map;

- $\mathcal{L}_{\tau_{0}}^{*}=L_{K / k, S}^{*}(0)^{\#}$;

- $\chi\left(\tau_{0}\right)=0$ if and only if $\operatorname{ETNC}\left(\mathbb{Q}(0)_{K}, \mathbb{Z}[G]\right)$ is valid.

Proof. The construction of [22, Prop. 3.1] defines a canonical object $\Psi_{S}$ of $D^{\text {perf }}(\mathbb{Z}[G])$ that is acyclic outside degrees 0 and 1 . Indeed, whilst the definition of $\Psi_{S}$ in [22] explicitly mentions the assumption $\operatorname{Pic}\left(\mathcal{O}_{K, S}\right)=0$, it is straightforward to check that if $\operatorname{Pic}\left(\mathcal{O}_{K, S}\right) \neq 0$, then the methods of [22] define objects $\Psi_{S}$ and $\tilde{\Psi}_{S}$ of $D(\mathbb{Z}[G])$ which satisfy all properties described in [22, Prop. 3.1] (with the exception that in $[22,(29)]$ the term $X_{S}$ is replaced by a $G$-module which is a 1-extension of $X_{K, S}$ by $\left.\operatorname{Pic}\left(\mathcal{O}_{K, S}\right)\right)$ and also lie in exact triangles of the form $[22,(31),(32),(85)]$.

To define $\tau:=\tau_{0}$ we first set $H_{\tau}^{i}:=H^{i}\left(\Psi_{S}\right)$ for $i=0,1$ and then let $\epsilon_{\tau}$ denote the element of $\operatorname{Ext}_{\mathbb{Z}[G]}^{2}\left(H_{\tau}^{1}, H_{\tau}^{0}\right)$ that is represented by $\left(\Psi_{S}, \operatorname{id}_{H^{0}\left(\Psi_{S}\right)}, \operatorname{id}_{H^{1}\left(\Psi_{S}\right)}\right)$ in the sense of $\S 3.1$. We also define $\lambda_{\tau}$ and $\mathcal{L}_{\tau}^{*}$ to be as in the statement of the Proposition. Then $[24,(29)]$ implies that

$$
\psi^{*}\left(T \Omega\left(\mathbb{Q}(0)_{K}, \mathbb{Z}[G]\right)\right)=\chi\left(\tau_{0}\right)
$$

and so $\operatorname{ETNC}\left(\mathbb{Q}(0)_{K}, \mathbb{Z}[G]\right)$ is indeed equivalent to the equality $\chi\left(\tau_{0}\right)=0$.

Remark 4.2.3. Weil-étale cohomology

In [18, Rem. 3.7] the 2-extension $\epsilon_{\tau_{0}}$ constructed above is interpreted in terms of Lichtenbaum's conjectural theory of the 'Weil-étale cohomology with compact support' of $\mathbb{Z}$ on $\operatorname{Spec}\left(\mathcal{O}_{K, S}\right)$.

Remark 4.2.4. Tate sequences 
In $[16, \S 2.3]$ it is shown that the conjectural vanishing of $\chi\left(\tau_{0}\right)$ is equivalent to the 'Lifted Root Number Conjecture' formulated by Gruenberg, Ritter and Weiss in [58]. In the same direction, Parker [81] has recently shown that the complex $\Psi_{S}$ used in the proof of Proposition 4.2.2 can be interpreted as a 'Tate sequence' in the sense described by Ritter and Weiss in [83].

4.2.2. $\mathbb{Q}(1)_{K}$. We fix a finitely generated projective $\mathbb{Z}[G]$-submodule $\mathcal{L}$ of $\mathcal{O}_{K}$ such that for each non-archimedean place $v$ in $S$ the exponential map induces a well defined injective homomorphism $\exp _{\mathcal{L}, v}: \mathcal{L}_{v} \rightarrow K_{v}^{\times}$. We let $\operatorname{Tr}_{\mathcal{L}}: \mathcal{L} \rightarrow \mathbb{Z}$ denote the restriction of the trace map $\operatorname{Tr}_{K / \mathbb{Q}}: K \rightarrow \mathbb{Q}$. We write $\exp _{\mathcal{L}, S}$ for the composite $\prod_{v \in S} \mathcal{L}_{v} \rightarrow \prod_{v \in S} K_{v}^{\times} \rightarrow C_{S}(K)$ where the first arrow is $\prod_{v \in S} \exp _{\mathcal{L}, v}$ (with $\mathcal{L}_{v}=K_{v}$ if $v$ is archimedean), $C_{S}(K)$ is the quotient of the idele class group of $K$ by the subgroup $\prod_{v \notin S} \mathcal{O}_{K_{v}}^{\times}$(this quotient is the group belonging to the $S$-class formation discussed in [74, Chap. I, §4]) and the second arrow is the natural projection. We use the isomorphism

$$
\lambda_{S}: \mathbb{R} \otimes \operatorname{ker}\left(\exp _{\mathcal{L}, S}\right) \cong \mathbb{R} \otimes_{\mathbb{Q}} \operatorname{ker}\left(\operatorname{Tr}_{K / \mathbb{Q}}\right)
$$

induced by the natural projection $\prod_{v \in S} \mathcal{L}_{v} \rightarrow \mathbb{R} \otimes_{\mathbb{Q}} K$ (cf. [13, Lem. 3.1(iii)]).

Proposition 4.2.5. There exists an a.t.e. of $\mathbb{Z}[G]$-modules $\tau_{1}$ with all of the following properties:-

- $\left(H_{\tau_{1}}^{0}\right)_{\text {tor }}=\operatorname{cok}\left(\operatorname{Tr}_{\mathcal{L}}\right)^{\vee},\left(H_{\tau_{1}}^{0}\right)_{\mathrm{tf}}=\operatorname{ker}\left(\operatorname{Tr}_{\mathcal{L}}\right)^{*},\left(H_{\tau_{1}}^{1}\right)_{\text {tor }}=\operatorname{cok}\left(\exp _{\mathcal{L}, S}\right)^{\vee}$ and $\left(H_{\tau_{1}}^{1}\right)_{\mathrm{tf}}=\operatorname{ker}\left(\exp _{\mathcal{L}, S}\right)^{*}$

- $\lambda_{\tau_{1}}$ is equal to the $\mathbb{R}$-linear dual of $\lambda_{S}$;

- $\mathcal{L}_{\tau_{1}}^{*}=L_{K / k, S}^{*}(1)^{\#}$;

- If there exists a finite Galois extension $K^{\prime}$ of $\mathbb{Q}$ with $K^{\prime}$ complex, $K \subseteq K^{\prime}$ and such that $K^{\prime}$ validates Leopoldt's conjecture, then $\chi\left(\tau_{1}\right)=0$ if and only if $\operatorname{ETNC}\left(\mathbb{Q}(1)_{K}, \mathbb{Z}[G]\right)$ is valid.

Proof. We recall that $\left[13\right.$, Lem. 3.1] defines an object $E_{S}(\mathcal{L})$ of $D^{\text {perf }}(\mathbb{Z}[G])$ and we set $C^{\bullet}:=R \operatorname{Hom}_{\mathbb{Z}}\left(E_{S}(\mathcal{L}), \mathbb{Z}\right)$ and $H_{\tau_{1}}^{i}:=H^{i}\left(C^{\bullet}\right)$ for $i=0,1$. It is clear that $C^{\bullet}$ belongs to $D^{\text {perf }}(\mathbb{Z}[G])$. Also, in each degree $i$ the universal coefficient spectral sequence gives natural isomorphisms $H^{i}\left(C^{\bullet}\right)_{\text {tor }} \cong\left(H^{-i+1}\left(E_{S}(\mathcal{L})\right)_{\text {tor }}\right)^{\vee}$ and $H^{i}\left(C^{\bullet}\right)_{\mathrm{tf}} \cong H^{-i}\left(E_{S}(\mathcal{L})\right)^{*}$. The explicit descriptions of $H_{\tau_{1}}^{0}$ and $H_{\tau_{1}}^{1}$ given above thus follow from the descriptions of the groups $H^{i}\left(E_{S}(\mathcal{L})\right)$ in [13, proof of Lem. 3.1]. The same argument shows that $C^{\bullet}$ is acyclic outside degrees 0 and 
1 and hence that $C^{\bullet}$ corresponds to a canonical element $\epsilon_{\tau_{1}}$ of $\operatorname{Ext}_{G}^{2}\left(H_{\tau_{1}}^{1}, H_{\tau_{1}}^{0}\right)$. Thus, if we define $\lambda_{\tau_{1}}$ and $\mathcal{L}_{\tau_{1}}^{*}$ as in the statement of the Proposition, and use the element $T \Omega(K / k, 1)=\left[E_{S}(\mathcal{L}), \lambda_{S}^{-1}\right]+\delta\left(L_{K / k, S}^{*}(1)\right)$ defined in $[13, \S 3.2]$, then (5) implies that

$$
\psi^{*}(T \Omega(K / k, 1))=\left[\epsilon_{\tau_{1}}, \lambda_{\tau_{1}}\right]-\delta\left(L_{K / k, S}^{*}(1)^{\#}\right)=\chi\left(\tau_{1}\right) .
$$

The final claim of the Proposition thus follows from the equality $T \Omega(K / k, 1)=$ $T \Omega\left(\mathbb{Q}(1)_{K}, \mathbb{Z}[G]\right)$ that is proved (under the stated hypotheses) by Breuning and the present author in [14].

4.2.3. $\mathbb{Q}(r)_{K}$ with $r<0$. In this case, for each odd prime $\ell$ and integer $i=1,2$ the Quillen-Lichtenbaum Conjecture predicts the existence of natural isomorphisms [70, Conj. 2.5]

$$
\operatorname{ch}_{\ell, r}^{i}: \mathbb{Z}_{\ell} \otimes K_{2-i-2 r}\left(\mathcal{O}_{K, S}\right) \rightarrow H_{\text {ét }}^{i}\left(\operatorname{Spec}\left(\mathcal{O}_{K, S}\left[\frac{1}{\ell}\right]\right), \mathbb{Z}_{\ell}(r)\right),
$$

where $K_{*}\left(\mathcal{O}_{K, S}\right)$ denotes Quillen's $K$-theory of the ring $\mathcal{O}_{K, S}$. Such homomorphisms have been constructed by Soulé [89] and by Dwyer and Friedlander [41] and proven to be surjective and to have finite kernel. Soulé used higher Chern class maps, which approximate the $\operatorname{maps}_{\ell, r}^{i}$, and Dwyer-Friedlander used étale $K$-theory. In the case $\ell=2 \operatorname{maps} \operatorname{ch}_{2, r}^{i}$ as above have been constructed and studied by Kahn [63] and by Rognes and Weibel [84] and have been shown to be bijective if, for example, $K$ is totally imaginary (and shown not to be bijective in general).

In the following result we set $\mathbb{Z}^{\prime}:=\mathbb{Z}\left[\frac{1}{2}\right]$.

Proposition 4.2.6. Let $r$ be a strictly negative integer. If the Chern class maps in (10) are bijective for all odd primes $\ell$, then there exists an a.t.e. of $\mathbb{Z}[G]$ modules $\tau_{r}$ with all of the following properties:-

- If $K$ is totally imaginary, then $H_{\tau_{r}}^{0}=K_{1-2 r}\left(\mathcal{O}_{K}\right)$ and $\left(H_{\tau_{r}}^{1}\right)_{\text {tor }}=K_{-2 r}\left(\mathcal{O}_{K, S}\right)$ and $\left(H_{\tau_{r}}^{1}\right)_{\mathrm{tf}}=\left(\bigoplus_{\mathrm{Hom}(K, \mathbb{C})}(2 \pi i)^{-r} \mathbb{Z}\right)^{\mathrm{Gal}(\mathbb{C} / \mathbb{R})}$ where in the last term $\operatorname{Gal}(\mathbb{C} / \mathbb{R})$ acts diagonally on the direct sum. This description of $H_{\tau_{r}}^{0}$, resp. $\left(H_{\tau_{r}}^{1}\right)_{\text {tor }}$, is also valid in any case for which $r \equiv 0,1(\bmod 4)$, resp. $r \equiv 0,3$ $(\bmod 4)$, and in all cases one has $\mathbb{Z}^{\prime} \otimes H_{\tau_{r}}^{0}=\mathbb{Z}^{\prime} \otimes K_{1-2 r}\left(\mathcal{O}_{K}\right)$ and $\mathbb{Z}^{\prime} \otimes$ $\left(H_{\tau_{r}}^{1}\right)_{\text {tor }}=\mathbb{Z}^{\prime} \otimes K_{-2 r}\left(\mathcal{O}_{K, S}\right)$ and $\mathbb{Z}^{\prime} \otimes\left(H_{\tau_{r}}^{1}\right)_{\mathrm{tf}}=\left(\bigoplus_{\mathrm{Hom}(K, \mathbb{C})}(2 \pi i)^{-r} \mathbb{Z}^{\prime}\right)^{\mathrm{Gal}(\mathbb{C} / \mathbb{R})}$;

- $\lambda_{\tau_{r}}$ is induced by -1 times the Beilinson regulator map (as described in $[15])$; 
- $\mathcal{L}_{\tau_{r}}^{*}=L_{K / k, S}^{*}(r)^{\#}$;

- $\chi\left(\tau_{r}\right)=0$ if and only if $\operatorname{ETNC}\left(\mathbb{Q}(r)_{K}, \mathbb{Z}[G]\right)$ is valid.

Proof. See $\S 11$.

\section{Remark 4.2.7. The Quillen-Lichtenbaum Conjecture}

If $\ell$ is odd, then the bijectivity of $\operatorname{ch}_{\ell,-1}^{1}$, resp. $\operatorname{ch}_{\ell,-1}^{2}$, has been proved by Levine [69] and Merkuriev and Suslin [75], resp. by Tate [93]. The more recent (2-adic) work of Kahn [63] and of Rognes and Weibel [84, 85] depends upon the fundamental work of Voevodsky, Suslin and Rost on the Milnor and Bloch-Kato conjectures and it seems widely believed that these methods should extend to prove the bijectivity of $\operatorname{ch}_{\ell, r}^{i}$ for all odd $\ell$, all strictly negative $r$ and both $i=1,2$.

Remark 4.2.8. $\mathbb{Q}(r)_{K}$ with $r>1$

If $r$ is any integer with $r>1$, then it remains an open problem to construct an a.t.e. of $\mathbb{Z}[G]$-modules $\tau_{r}$ for which both $\mathcal{L}_{\tau_{r}}^{*}=L_{K / k, S}^{*}(r)^{\#}$ and $\chi\left(\tau_{r}\right)=0$ if and only if $\operatorname{ETNC}\left(\mathbb{Q}(r)_{K}, \mathbb{Z}[G]\right)$ is valid.

4.3. Elliptic curves. Let $E$ be an elliptic curve that is defined over $\mathbb{Q}$ and $K$ a finite Galois extension of $\mathbb{Q}$ for which the Tate-Shafarevic group $\amalg\left(E_{/ K}\right)$ of $E$ over $K$ is finite. We set $G:=\operatorname{Gal}(K / \mathbb{Q})$ and write $\operatorname{disc}(K)$ for the discriminant of $K / \mathbb{Q}$, $\operatorname{cond}(E)$ for the conductor of $E$ and $\operatorname{Sel}\left(E_{/ K}\right)$ for the 'integral Selmer group' of $E_{/ K}$ that is defined by Mazur and Tate in $[73$, p. 720]. We recall in particular that there is a canonical exact sequence of $G$-modules

$$
0 \rightarrow \amalg\left(E_{/ K}\right)^{\vee} \rightarrow \operatorname{Sel}\left(E_{/ K}\right) \rightarrow \operatorname{Hom}_{\mathbb{Z}}(E(K), \mathbb{Z}) \rightarrow 0
$$

where $E(K)$ is the Mordell-Weil group of $E_{/ K}$ and therefore also canonical isomorphisms $\operatorname{Sel}\left(E_{/ K}\right)_{\text {tor }} \cong \amalg\left(E_{/ K}\right)^{\vee}$ and $\operatorname{Sel}\left(E_{/ K}\right)_{\mathrm{tf}} \cong \operatorname{Hom}_{\mathbb{Z}}(E(K), \mathbb{Z})$.

We write $S_{\text {ram }}$ for the (finite) set of prime divisors of $\operatorname{disc}(K)$ and for each character $\psi$ in $\operatorname{Irr}(G)$ we let $L_{S_{\text {ram }}}^{*}(E, \psi, 1)$ denote the leading term in the Taylor expansion at $s=1$ of the $S_{\text {ram }}$-truncated $\psi$-twisted Hasse-Weil $L$-function $L_{S_{\text {ram }}}(E, \psi, s)$. For each such $\psi$ and each prime $\ell$ in $S_{\text {ram }}$ we also define, following [49, Chap. IV, §1], a 'non-ramified characteristic' element $n_{\ell, \psi}$ of $\mathbb{Q}^{c \times}$ in the following way. We fix a place $w$ of $K$ above $\ell$ and write $G_{w}$ and $I_{w}$ for the decomposition and inertia subgroups of $w$ in $G$ and $\operatorname{Fr}_{w}$ for the Frobenius automorphism in $G_{w} / I_{w}$. For each $\chi \in \operatorname{Irr}\left(G_{w}\right)$ we fix a representation space $V_{\chi}$ 
of character $\chi$ and then for each $\psi \in \operatorname{Irr}(G)$ we set

$$
n_{\ell, \psi}:= \begin{cases}\operatorname{det}\left(-\mathrm{Fr}_{w} \mid\left(V_{\operatorname{res}_{G_{w}}^{G} \psi}\right)^{I_{w}}\right), & \text { if }\left(V_{\operatorname{res}_{G}^{G} \psi}\right)^{I_{w}} \neq 0 \\ 1, & \text { otherwise. }\end{cases}
$$

For each character $\psi$ in $\operatorname{Irr}(G)$ we then define a 'modified Galois-Gauss sum' by setting

$$
\tau^{*}(\psi):=\tau(\psi) \prod_{\ell \in S_{\mathrm{ram}}} n_{\ell, \psi}^{-1}
$$

where $\tau(\psi)$ is the Galois-Gauss sum defined in [49, Chap. I, §5].

We also set $\Omega(E):=\left|\int_{\gamma^{+}} \omega\right|$ where $\gamma^{+}$is a generator of the submodule of $H_{1}(E(\mathbb{C}), \mathbb{Z})$ fixed by complex conjugation and $\omega$ is a Néron differential.

For each prime $\ell$ we write $\tilde{E}_{\ell}$ for the reduction of the minimal model of $E$ at $\ell$ and $\mathbb{F}_{\ell}$ for the finite field of cardinality $\ell$. If $\ell$ divides $\operatorname{cond}(E)$, then for each subfield $F$ of $K$ that is Galois over $\mathbb{Q}$ we write $c_{\ell}(E, F)$ for the Tamagawa factor at $\ell$ of the pair $\left(h^{1}\left(E_{/ F}\right)(1), \mathbb{Z}[\operatorname{Gal}(F / \mathbb{Q})]\right.$ ) (for a definition of this nonzero integer see $\S 12.1)$. For each prime $\ell$ in $S_{\text {ram }}$ we also write $P_{\ell}(E, K)$ for the element of $\zeta(\mathbb{Q}[G])^{\times}$given by the value at $s=1$ of the equivariant Euler factor $\left(L_{\ell}(E, \psi, s)\right)_{\psi}$ at $\ell$, where $\psi$ runs over $\operatorname{Irr}(G)$.

Proposition 4.3.1. Let $E$ be an elliptic curve defined over $\mathbb{Q}$. Let $K$ be a finite totally real Galois extension of $\mathbb{Q}$ and $\Lambda$ a finitely generated subring of $\mathbb{Q}$ in which 2 is invertible and assume that at least one of the following conditions (A) and (B) is satisfied.

(A) Any prime which either divides the degree of $K / \mathbb{Q}$, or divides both $\operatorname{disc}(K)$ and $\operatorname{cond}(E)$, is invertible in $\Lambda$.

(B) All of the following conditions (i)-(v) are satisfied:

(i) The degree of $K / \mathbb{Q}$ is a power of an odd prime $p$;

(ii) $\operatorname{disc}(K)$ is coprime to both $p$ and $\operatorname{cond}(E)$;

(iii) $p \nmid\left|E(\mathbb{Q})_{\text {tor }}\right| \prod_{\ell \mid \operatorname{disc}(K)}\left|\tilde{E}_{\ell}\left(\mathbb{F}_{\ell}\right)\right|$;

(iv) $p \nmid \operatorname{cond}(E)$;

(v) $p \nmid \prod_{\ell \mid \operatorname{cond}(E)} c_{\ell}(E, \mathbb{Q})$.

Set $G:=\operatorname{Gal}(K / \mathbb{Q})$. Then then there exists an a.t.e. of $\Lambda[G]$-modules $\tau_{E, \Lambda}$ with all of the following properties:- 
- One has

$$
\begin{aligned}
& H_{\tau_{E, \Lambda}}^{0}=(\Lambda \otimes E(K)) \oplus\left(\Lambda \otimes\left(E(K)_{\text {tor }}\right)^{\vee}\right) \oplus c_{E, \Lambda}^{0}(K), \\
& H_{\tau_{E, \Lambda}}^{1}=\left(\Lambda \otimes \operatorname{Sel}\left(E_{/ K}\right)\right) \oplus c_{E, \Lambda}^{1}(K)
\end{aligned}
$$

where $c_{E, \Lambda}^{0}(K)$ and $c_{E, \Lambda}^{1}(K)$ are finite $\Lambda[G]$-modules which satisfy the following condition: if $q$ is any prime divisor of $\left|c_{E}^{0}(K)\right|\left|c_{E}^{1}(K)\right|$, then either $q$ divides $\operatorname{disc}(K) \operatorname{cond}(E) \prod_{\ell \mid \operatorname{cond}(E)} c_{\ell}(E, K)$ or for some prime divisor $\ell$ of $\operatorname{disc}(K)$ the image of $P_{\ell}(E, K)$ in $\zeta\left(\mathbb{Q}_{q}[G]\right)^{\times} \cong K_{1}\left(\mathbb{Q}_{q}[G]\right)$ does not belong to the image of the natural map $K_{1}\left(\mathbb{Z}_{q}[G]\right) \rightarrow K_{1}\left(\mathbb{Q}_{q}[G]\right)$. If condition $(B)$ is satisfied, then also $p$ does not divide $\left|E(K)_{\text {tor }}\right|,\left|c_{E, \Lambda}^{0}(K)\right|$ or $\left|c_{E, \Lambda}^{1}(K)\right|$.

- $\lambda_{\tau_{E, \Lambda}}$ is induced by the Néron-Tate height pairing.

- $\left(\mathcal{L}_{\tau_{E, \Lambda}}^{*}\right)_{\psi}=\Omega(E)^{-\psi(1)} \tau^{*}(\psi) L_{S_{\mathrm{ram}}}^{*}(E, \bar{\psi}, 1)$ for each $\psi$ in $\operatorname{Irr}(G)$.

- $\chi\left(\tau_{E, \Lambda}\right)$ vanishes if and only if $\operatorname{ETNC}\left(h^{1}\left(E_{/ K}\right)(1), \Lambda[G]\right)$ is valid.

Proof. See $\S 12$.

Remark 4.3.2. Conditions $(A)$ and $(B)$

The significance of condition (A) in Proposition 4.3.1 is that, for a given elliptic curve $E$, it shows that our techniques can be used to derive explicit consequences of $\operatorname{ETNC}\left(h^{1}\left(E_{/ K}\right)(1), \Lambda[G]\right)$ for any totally real Galois extension $K / \mathbb{Q}$ for which $\amalg\left(E_{/ K}\right)$ is finite. The more restrictive condition (B) is important because, for a given $E$, it shows that there are natural families of extensions $K / \mathbb{Q}$ for which our techniques can be used to derive explicit consequences of $\operatorname{ETNC}\left(h^{1}\left(E_{/ K}\right)(1), \mathbb{Z}\left[\frac{1}{2}\right][G]\right)$ and working with $\mathbb{Z}\left[\frac{1}{2}\right][G]$ (rather than with $\Lambda[G]$ for a ring $\Lambda$ in which $|G|$ is invertible) is essential, for example, when dealing with the $G$-valued height pairings we discuss in $\S 9$. In this regard we reassure the reader that for any given elliptic curve $E$ there are infinitely many primes $p$ and extensions $K / \mathbb{Q}$ which together satisfy all of the conditions listed in (B) above. To explain this we first fix a finite set $\Sigma$ of primes which does not contain any prime divisor of $\operatorname{cond}(E)$ and consider the condition

$\left(\mathrm{iii}^{\prime}\right) p \nmid 2\left|E(\mathbb{Q})_{\text {tor }}\right| \prod_{\ell \in \Sigma}\left|\tilde{E}_{\ell}\left(\mathbb{F}_{\ell}\right)\right|$.

Then it is clear that conditions (iii'), (iv) and (v) rule out only finitely many $p$. Hence, if we fix any prime $p$ that does not belong to $\Sigma$ and satisfies conditions (iii'), (iv) and (v), then one can ensure that all of the stated conditions (i)-(v) are 
satisfied by simply choosing $K$ to be any totally real Galois extension of $\mathbb{Q}$ which is of $p$-power degree, unramified at $p$ and $\operatorname{such}$ that $\operatorname{disc}(K)$ is divisible only by primes that belong to $\Sigma$.

\section{Remark 4.3.3. Supporting evidence}

If $K=k$, then $\operatorname{ETNC}\left(h^{1}\left(E_{/ K}\right)(1), \mathbb{Z}[\operatorname{Gal}(K / k)]\right)$ is equivalent to the famous conjecture of Birch and Swinnerton-Dyer for $E_{/ K}$ (cf. [44, §5.4]). In the general case it is known that $\operatorname{ETNC}\left(h^{1}\left(E_{/ K}\right)(1), \mathbb{Z}[\operatorname{Gal}(K / k)]\right)$ is a consequence of (a natural refinement of) the 'main conjecture of non-commutative Iwasawa theory' formulated by Coates, Fukaya, Kato, Sujatha and Venjakob in [35] (cf. the Introduction). In addition to these compatibilities, Navilarekallu [77] and Bley [7] have recently obtained convincing numerical evidence in support of the conjecture for certain elliptic curves $E$ and certain non-abelian Galois extensions of $k=\mathbb{Q}$.

4.4. General motives. Modulo certain standard expectations the statement of $\operatorname{ETNC}\left(M_{K}, \Lambda[G]\right)$ should be equivalent to the vanishing of the Euler characteristic of a natural a.t.e. of $\Lambda[G]$-modules in far greater generality than already described in $\S 4.2$ and $\S 4.3$.

To explain this we first recall that to each motive $M$ over $k$ (in the sense of $\S 4.1)$ there is associated a finite dimensional 'motivic cohomology' $\mathbb{Q}$-vector space $H^{0}(k, M)$. For example, if $M=h^{n}(X)(r)$ as in $\S 4.1$ then one possible explicit definition of this space is

$$
H^{0}(k, M):= \begin{cases}\left(\mathrm{CH}^{r}(X) / \mathrm{CH}^{r}(X)_{\text {hom } \sim 0}\right) \otimes \mathbb{Q}, & \text { if } n=2 r \\ 0, & \text { otherwise }\end{cases}
$$

and for more general $M$ one expects that an appropriate generalisation will be given by defining $H^{0}(k, M)$ to be the group of homomorphisms from $\mathbb{Q}$ to $M$ in the 'category of mixed motives over $k$ '.

Then, since the category of motives $\mathcal{M}_{k}$ over $k$ is expected to be semisimple, for any $M$ in $\mathcal{M}_{k}$ there should exist an object $\hat{M}$ of $\mathcal{M}_{k}$ such that the spaces $H^{0}(k, \hat{M})$ and $H^{0}\left(k, \hat{M}^{*}(1)\right)$ both vanish and $M$ decomposes in $\mathcal{M}_{k}$ as a direct sum

$$
M \cong \mathbb{Q}(0)_{k}^{\operatorname{dim}_{\mathbb{Q}}\left(H^{0}(k, M)\right)} \oplus \mathbb{Q}(1)_{k}^{\operatorname{dim}_{\mathbb{Q}}\left(H^{0}\left(k, M^{*}(1)\right)\right)} \oplus \hat{M} .
$$

In view of Propositions 4.2.2 and 4.2.5, when attempting to explicate the statement of $\operatorname{ETNC}\left(M_{K}, \Lambda[G]\right)$, one can therefore assume that both of the spaces 
$H^{0}(k, M)$ and $H^{0}\left(k, M^{*}(1)\right)$ vanish. There are then two methods of applying the approach of $\S 3$ to such $M$. The first extends the approach of $\S 4.2 .3$ to motives whose tangent space is also trivial (see Remark 11.3.1). The second generalises the approach of $\S 4.3$. Indeed, whilst the latter approach extends directly to (suitable) abelian varieties, it can also be generalised to critical motives by replacing the 'finite support cohomology' used in $\S 12$ by the 'Selmer complexes' introduced by Nekováŕ in [78]. This aspect of the theory will be developed further elsewhere.

\section{Statement of the main algebraic Results}

Motivated by the discussion of $\S 4$, and in particular by the final assertions in each of Propositions 4.2.2, 4.2.5, 4.2.6 and 4.3.1, in $\S 6-\S 9$ we will investigate certain explicit consequences of the vanishing of the Euler characteristic of an arbitrary a.t.e. In each such case we will also discuss the (conjectural) consequences which thereby arise in the context of the arithmetic examples described in $\S 4$.

5.1. The general case. For the reader's convenience, in the next result we collect together some of the main algebraic results that are to be proved in $\S 6-\S 9$.

Theorem 5.1.1. Let $\Lambda$ be any finitely generated subring of $\mathbb{Q}$ and $\tau$ an augmented $\mathbb{R}$-trivialized extension of $\Lambda[G]$-modules for which the Euler characteristic $\chi(\tau)$ vanishes. Then each of the following assertions is valid.

(i) (Algebraicity) Fix a $\Lambda[G]$-module homomorphism $\varphi: H_{\tau}^{1} \rightarrow H_{\tau}^{0}$ for which $\mathbb{Q} \otimes_{\Lambda} \varphi$ is bijective. For each character $\psi \in \operatorname{Irr}(G)$ choose a complex vector space $V_{\psi}$ which realizes $\psi$ and set

$$
A_{\varphi}^{\tau}(\psi):=\left(\mathcal{L}_{\tau, \psi}^{*}\right)^{-1} \operatorname{det}_{\mathbb{C}}\left(\left(\mathbb{C} \otimes_{\mathbb{R}} \lambda_{\tau}\right) \circ\left(\mathbb{C} \otimes_{\Lambda} \varphi\right) \mid \operatorname{Hom}_{\mathbb{C}[G]}\left(V_{\psi}, \mathbb{C} \otimes_{\Lambda} H_{\tau}^{1}\right)\right) \in \mathbb{C}^{\times}
$$

Then for every $\psi \in \operatorname{Irr}(G)$ and $\omega \in \operatorname{Aut}_{\mathbb{Q}}(\mathbb{C})$ one has

$$
A_{\varphi}^{\tau}(\omega \circ \psi)=\omega\left(A_{\varphi}^{\tau}(\psi)\right)
$$

(ii) (Explicit Euler characteristics) Assume the notation of claim (i). Fix $\psi \in \operatorname{Irr}(G)$ and let $E$ be any subfield of $\mathbb{C}$ which is both Galois and of finite degree over $\mathbb{Q}$ and also large enough to ensure that, with $\mathcal{O}_{E, \Lambda}$ denoting the integral closure of $\Lambda$ in $E$, there exists a finitely generated $\mathcal{O}_{E, \Lambda}[G]$ lattice $T_{\psi}$ for which the $\mathbb{C}[G]$-module $\mathbb{C} \otimes_{\mathcal{O}_{E, \Lambda}} T_{\psi}$ is isomorphic to $V_{\psi}$. 
Then $A_{\varphi}^{\tau}(\psi)$ belongs to $E^{\times}$and in $E$ one has

$$
A_{\varphi}^{\tau}(\psi) \mathcal{O}_{E, \Lambda}=\operatorname{char}_{\mathcal{O}_{E, \Lambda}}\left(\operatorname{cok}\left(\varphi^{(\psi)}\right)\right) \operatorname{char}_{\mathcal{O}_{E, \Lambda}}\left(\operatorname{ker}\left(\varphi^{(\psi)}\right)\right)^{-1},
$$

where $\varphi^{(\psi)}$ denotes the composite homomorphism

$$
\begin{aligned}
\operatorname{Hom}_{\mathcal{O}_{E, \Lambda}}\left(T_{\psi}, \mathcal{O}_{E, \Lambda} \otimes_{\Lambda} H_{\tau}^{1}\right)_{G} \stackrel{\sum_{g \in G} g}{\longrightarrow} \operatorname{Hom}_{\mathcal{O}_{E, \Lambda}}\left(T_{\psi}, \mathcal{O}_{E, \Lambda} \otimes_{\Lambda} H_{\tau}^{1}\right)^{G} \\
\rightarrow \operatorname{Hom}_{\mathcal{O}_{E, \Lambda}}\left(T_{\psi}, \mathcal{O}_{E, \Lambda} \otimes_{\Lambda} H_{\tau}^{0}\right)^{G}
\end{aligned}
$$

Here the last arrow is induced by $\varphi$ and for any finite $\mathcal{O}_{E, \Lambda}$-module $\mathcal{F}$ we write $\operatorname{char}_{\mathcal{O}_{E, \Lambda}}(\mathcal{F})$ for the (unique) ideal of $\mathcal{O}_{E, \Lambda}$ which at every maximal ideal $\mathfrak{p}$ of $\mathcal{O}_{E, \Lambda}$ satisfies $\operatorname{char}_{\mathcal{O}_{E, \Lambda}}(\mathcal{F})_{\mathfrak{p}}=\left(\mathfrak{p} \mathcal{O}_{E, \mathfrak{p}}\right)^{\text {length }_{\mathcal{O}_{E, \mathfrak{p}}}\left(\mathcal{F}_{\mathfrak{p}}\right)}$.

(iii) (Galois module structures) If $\Psi^{0}$ and $\Psi^{1}$ are any finitely generated cohomologically-trivial $\Lambda[G]$-modules lying in a 2 -extension

$$
0 \rightarrow H_{\tau}^{0} \rightarrow \Psi^{0} \rightarrow \Psi^{1} \rightarrow H_{\tau}^{1} \rightarrow 0
$$

of class $\epsilon_{\tau}$, then the Euler characteristic

$$
\chi\left(\epsilon_{\tau}\right):=\left(\Psi^{0}\right)-\left(\Psi^{1}\right) \in K_{0}(\Lambda[G])
$$

depends only upon the signs of $\mathcal{L}_{\tau, \kappa}^{*}$ for each irreducible complex symplectic character $\kappa$ of $G$. In particular, one has

- $\chi\left(\epsilon_{\tau}\right)=0$ if $G$ has no irreducible complex symplectic characters,

- $2 \chi\left(\epsilon_{\tau}\right)=0$,

- $\left(\psi^{*}-1\right)\left(\chi\left(\epsilon_{\tau}\right)\right)=0$ where $\psi^{*}$ is the involution of $K_{0}(\Lambda[G])$ defined in $§ 2.5$.

(iv) (Strong main conjectures) Let $G$ be abelian, let $e_{\tau}$ denote the sum over all primitive idempotents of $\mathbb{C}[G]$ which annihilate $\mathbb{C} \otimes_{\Lambda} H_{\tau}^{0}$ and set

$$
\mathcal{L}_{\tau}:=\mathcal{L}_{\tau}^{*} e_{\tau} .
$$

Then the element $\mathcal{L}_{\tau}$ belongs to $\mathbb{Q}[G]$ and if $\left(H_{\tau}^{0}\right)_{\text {tor }}$ has finite projective dimension as a $\Lambda[G]$-module one also has

$$
\operatorname{Fit}_{\Lambda[G]}\left(\left(H_{\tau}^{0}\right)_{\text {tor }}\right) \cdot \mathcal{L}_{\tau}=\operatorname{Fit}_{\Lambda[G]}\left(H_{\tau}^{1}\right) .
$$

Here we write $\operatorname{Fit}_{\Lambda[G]}(M)$ for the (initial) Fitting ideal of any finitely generated $\Lambda[G]$-module $M$.

(v) (Explicit congruences) Let $G, \mathcal{L}_{\tau}$ and $\Lambda$ be as in claim (iv), fix an element $\alpha$ of $\operatorname{Fit}_{\Lambda[G]}\left(\left(H_{\tau}^{0}\right)_{\text {tor }}\right)$ and set

$$
n_{\tau}:=\operatorname{dim}_{\mathbb{Q}}\left(\mathbb{Q} \otimes_{\Lambda} H_{\tau, G}^{1}\right) .
$$


Then, with $I_{G, \Lambda}$ denoting the augmentation ideal of $\Lambda[G]$, one has

$$
\alpha \cdot \mathcal{L}_{\tau} \in I_{G, \Lambda}^{n_{\tau}}
$$

and the image of $\alpha \cdot \mathcal{L}_{\tau}$ in $\Lambda_{\alpha} \otimes_{\Lambda} I_{G, \Lambda}^{n_{\tau}}$ satisfies

$$
\alpha \cdot \mathcal{L}_{\tau} \equiv \operatorname{disc}_{\alpha}(\tau)\left(\bmod \Lambda_{\alpha} \otimes_{\Lambda} I_{G, \Lambda}^{n_{\tau}+1}\right)
$$

where $\Lambda_{\alpha}$ and $\operatorname{disc}_{\alpha}(\tau)$ are the subring of $\mathbb{Q}$ and the algebraic discriminant defined in (32) and (33) respectively.

\section{Remark 5.1.2. New conjectures}

In $\S 6-\S 9$ we will see that the predictions obtained by combining Theorem 5.1.1 with the results of $\S 4$ and the assumed validity of (relevant cases of) the Equivariant Tamagawa Number Conjecture recover a wide variety of well-known, and hitherto seemingly unrelated, conjectures that have been much studied in the literature. In several interesting cases we will also find that they give rise to new conjectures that are of a very explicit nature. For example, as far as we are aware, the conjectures discussed in each of Remarks 5.1.5, 6.2.4 (with $\tau=\tau_{1}$ ), 6.2.5, 7.1.5, 8.2.4, 8.2.5, 8.2.6, 9.5.3 and 9.5.4 and in Propositions 7.2.3(ii), 7.2.5 and 7.2.6(ii) and (iii) are new.

\section{Remark 5.1.3. The ETNC}

The arguments in $\S 6$ will show that if one applies Theorem 5.1.1 in the setting of the examples of $\S 4$, then the relations described in claims (i) and (ii) follow from the validity of $\operatorname{ETNC}\left(M_{K}, \mathfrak{M}\right)$ where $\mathfrak{M}$ is any choice of maximal order in $\mathbb{Q}[G]$ with $\Lambda[G] \subseteq \mathfrak{M}$ (see Remark 6.2.2). However, the relations described in claims (iii), (iv) and (v) of Theorem 5.1.1 and also in Theorem 5.2.1(iv) below are in general finer and do not follow from $\operatorname{ETNC}\left(M_{K}, \mathfrak{M}\right)$ for any such $\mathfrak{M}$.

Remark 5.1.4. Explicit congruences

Since $I_{G, \Lambda} \subseteq \Lambda[G]$, the containment $\alpha \cdot \mathcal{L}_{\tau} \in I_{G, \Lambda}^{n_{\tau}}$ in Theorem 5.1.1(v) provides an explicit bound on the 'denominator' of the element $\mathcal{L}_{\tau}$ of $\mathbb{Q}[G]$. Further, given the definition of $\operatorname{disc}_{\alpha}(\tau)$ in $\S 9.2$, the displayed congruence in Theorem 5.1.1(v) amounts to an explicit congruence relation between $\mathcal{L}_{\tau}^{*} e_{\tau}$ and $\mathcal{L}_{\tau}^{*} e_{G}$, where $e_{G}:=|G|^{-1} \sum_{g \in G} g$ is the idempotent of $\mathbb{Q}[G]$ associated to the trivial character of $G$. With further effort, our approach shows that the vanishing of $\chi(\tau)$ implies more general congruence relations between the different components 
of $\mathcal{L}_{\tau}^{*}$ (cf. Remark 9.5.4) and it seems likely that such congruences can be related to congruences of the form studied by Kato in [66].

Remark 5.1.5. Special elements

We use the notation of Theorem 5.1.1(i) and (ii), we assume that $\psi$ is such that $\operatorname{dim}_{\mathbb{C}}\left(\operatorname{Hom}_{\mathbb{C}[G]}\left(V_{\psi}, \mathbb{C} \otimes_{\Lambda} H_{\tau}^{1}\right)\right)=1$ and we fix an element $x$ of $H_{\tau, \mathrm{tf}}^{1}$. In this case it can be shown that if the equality $A_{\varphi}^{\tau}(\omega \circ \psi)=\omega\left(A_{\varphi}^{\tau}(\psi)\right)$ of Theorem 5.1.1(i) is valid for every $\omega \in \operatorname{Aut}_{\mathbb{Q}}(\mathbb{C})$, then for each element $d$ of $E$, there exists a unique element $\epsilon(\psi, x, d)$ of $\mathbb{Q} \otimes_{\Lambda} H_{\tau}^{0}$ such that in $\mathbb{R} \otimes_{\Lambda} H_{\tau}^{1}$ one has

$$
\lambda_{\tau}(\epsilon(\psi, x, d))=\sum_{\gamma \in \operatorname{Gal}(E / \mathbb{Q})} \sum_{g \in G} \gamma(d)(\gamma \circ \psi)(g) \mathcal{L}_{\tau, \psi^{\gamma}}^{*} \cdot g(x) .
$$

It can also be shown that the validity of (12) translates into an explicit upper bound on the denominator of $d$ which ensures that $\epsilon(\psi, x, d)$ belongs to the sublattice $H_{\tau, \mathrm{tf}}^{0}$ of $\mathbb{Q} \otimes_{\Lambda} H_{\tau}^{0}$. Now if $\tau=\tau_{0}$ as in Proposition 4.2.2, $k=\mathbb{Q}$ and $\psi$ has degree one, then the elements $\epsilon(\psi, x, d)$ of $H_{\tau, \mathrm{tf}}^{0}=\left(\mathcal{O}_{K, S}^{\times}\right)_{\mathrm{tf}}$ can be constructed explicitly by using cyclotomic elements. Also, if $\tau=\tau_{0}, k=\mathbb{Q}$ and $\psi$ is an odd irreducible complex representation of $\operatorname{Gal}\left(\mathbb{Q}^{c} / \mathbb{Q}\right)$ that is of degree two, then it is shown in [20] that this approach specialises to predict the existence of the 'Stark units' in $\mathcal{O}_{K}^{\times}$that are discussed by Stark in [90] and conjectured to exist by Chinburg in [30, Conj. 1]. However, if the degree of the irreducible character $\psi$ is greater than one and $\tau=\tau_{r}$ as in Proposition 4.2.6, resp. $\tau=\tau_{E, \Lambda}$ as in Proposition 4.3.1, then the predicted existence of special elements $\epsilon(\psi, x, d)$ of $\mathbb{Z}\left[\frac{1}{2}\right] \otimes H_{\tau, \mathrm{tf}}^{0}=\mathbb{Z}\left[\frac{1}{2}\right] \otimes K_{1-2 r}\left(\mathcal{O}_{K}\right)_{\mathrm{tf}}$, resp. of $H_{\tau, \mathrm{tf}}^{0}=\Lambda \otimes E(K)_{\mathrm{tf}}$, which are explicitly related to derivatives of $L$-functions via the above displayed formula is new and will be considered in detail elsewhere. For another prediction concerning the existence of modules of 'special elements' see Theorem 5.2.1(iv)(c).

5.2. The cyclic case. In addition to the general results described in Theorem 5.1.1 in certain special cases our methods can also give structural information of a very explicit nature. To state an example we recall that if $G$ is abelian, then a $\mathbb{Z}_{p}[G]$-module is said to be a 'permutation lattice' if it is isomorphic to a module of the form

$$
\bigoplus_{J} \mathbb{Z}_{p}[G / J]^{\left\langle n_{J}\right\rangle}
$$

where $J$ runs over the subgroups of $G$, each $n_{J}$ is a non-negative integer and $\mathbb{Z}_{p}[G / J]^{\left\langle n_{J}\right\rangle}$ denotes the direct sum of $n_{J}$ copies of $\mathbb{Z}_{p}[G / J]$. 
We recall that if $\mathfrak{A}$ is an order in $\mathbb{Q}[G]$, then a finitely generated $\mathfrak{A}$-module $N$ is said to be 'locally-free' if for every prime $p$ the associated $\mathbb{Z}_{p} \otimes \mathfrak{A}$-module $\mathbb{Z}_{p} \otimes N$ is free (and of rank independent of $p$ ).

For each subgroup $J$ of $G$ we write $e_{J}$ for the idempotent $|J|^{-1} \sum_{g \in J} g$ of $\mathbb{Q}[G]$.

Theorem 5.2.1. Let $G$ be a cyclic group of p-power order for some prime $p$ and $\Lambda$ a finitely generated subring of $\mathbb{Q}$ in which $p$ is not invertible. Let $\tau$ be an a.t.e. of $\Lambda[G]$-modules and set $H^{i}:=H_{\tau}^{i}$ for $i=0,1$. Assume that $H_{\mathrm{tor}}^{0}$ is a cohomologically-trivial $G$-module and that for every non-trivial subgroup $J$ of $G$ the module of coinvariants $\mathbb{Z}_{p} \otimes_{\Lambda} H_{J}^{1}$ is torsion-free.

(i) Then $\mathbb{Z}_{p} \otimes_{\Lambda} H_{\mathrm{tf}}^{0}$ is a permutation lattice.

(ii) The endomorphism ring $\operatorname{End}_{\mathbb{Q}[G]}\left(H_{\mathrm{tf}}^{0}\right)$ is generated over $\Lambda[G]$ by the set $\left\{e_{J}: J \leq G\right.$ and $\left.H_{\mathrm{tf}}^{0}=\left(H_{\mathrm{tf}}^{0}\right)^{J}\right\}$.

(iii) The following conditions are equivalent:-

(a) $\operatorname{dim}_{\mathbb{C}}\left(e_{\chi}\left(\mathbb{C} \otimes_{\Lambda} H^{0}\right)\right)$ is independent of $\chi \in \operatorname{Hom}\left(G, \mathbb{C}^{\times}\right)$;

(b) $\operatorname{dim}_{\mathbb{C}}\left(e_{\chi}\left(\mathbb{C} \otimes_{\Lambda} H^{1}\right)\right)$ is independent of $\chi \in \operatorname{Hom}\left(G, \mathbb{C}^{\times}\right)$;

(c) $H_{\mathrm{tf}}^{0}$ is a locally-free $\Lambda[G]$-module;

(d) $H^{0}$ is a cohomologically-trivial G-module;

(e) $H^{1}$ is a cohomologically-trivial G-module;

(f) $H_{\mathrm{tf}}^{0}$ is a locally-free $\operatorname{End}_{\mathbb{Q}[G]}\left(H_{\mathrm{tf}}^{0}\right)$-module.

(iv) Assume that the conditions of claim (iii) are satisfied and that the Euler characteristic $\chi(\tau)$ vanishes.

(a) In $K_{0}(\Lambda[G])$ one has $\left(H^{0}\right)=\left(H^{1}\right)$.

(b) Let $\Lambda^{\prime}$ be a finitely generated subring of $\mathbb{Q}$ which contains $\Lambda$ and is such that $\Lambda^{\prime} \otimes_{\Lambda} H_{\text {tor }}^{0}$ and $\Lambda^{\prime} \otimes_{\Lambda} H_{\text {tor }}^{1}$ both vanish. Then the $\Lambda^{\prime}[G]$ modules $\Lambda^{\prime} \otimes_{\Lambda} H^{0}$ and $\Lambda^{\prime} \otimes_{\Lambda} H^{1}$ are isomorphic.

(c) Let $d$ be the (common) rank of the free $\mathbb{Q}[G]$-modules $\mathbb{Q} \otimes_{\Lambda} H^{0}$ and $\mathbb{Q} \otimes_{\Lambda} H^{1}$. If the $G$-module $H_{\text {tor }}^{1}$ is cohomologically-trivial, then there exists a free rank one $\Lambda[G]$-submodule $\mathcal{E}$ of $\wedge_{\Lambda[G]}^{d} H_{\mathrm{tf}}^{0}$ which satisfies both

$$
\begin{aligned}
& \mathbb{Z}_{(p)} \otimes_{\Lambda}\left(\wedge_{\mathbb{R}[G]}^{d} \lambda_{\tau}\right)(\mathcal{E})=\mathbb{Z}_{(p)} \otimes_{\Lambda}\left(\mathcal{L}_{\tau}^{*} \cdot \operatorname{Fitt}_{\Lambda[G]}\left(H_{\text {tor }}^{0}\right) \wedge_{\Lambda[G]}^{d} H_{\mathrm{tf}}^{1}\right) \\
& \quad \text { and } \\
& \mathbb{Z}_{(p)} \otimes_{\Lambda} \operatorname{Ann}_{\Lambda[G]}\left(\left(\wedge_{\Lambda[G]}^{d} H_{\mathrm{tf}}^{0}\right) / \mathcal{E}\right)=\mathbb{Z}_{(p)} \otimes_{\Lambda} \operatorname{Fitt}_{\Lambda[G]}\left(H_{\mathrm{tor}}^{1}\right) .
\end{aligned}
$$




\section{Algebraicity And explicit Euler Characteristic formulas}

In this section we prove claims (i) and (ii) of Theorem 5.1.1 and also discuss arithmetic applications of these results in the setting of the examples described in $\S 4$. The argument in this section is an abstraction of that given by Flach and the present author in $[24, \S 3]$ and is therefore closely modelled on previous work of Stark, Tate [94], Gross [56], Chinburg [32] and Chinburg, Kolster, Pappas and Snaith [33].

At the outset we recall that a character $\kappa$ in $\operatorname{Irr}(G)$ is said to be 'symplectic' if the subfield $E_{\kappa}$ of $\mathbb{C}$ that is generated by the values of $\kappa$ is totally real and $\operatorname{End}_{\mathbb{R}[G]}\left(V_{\kappa}\right)$ is isomorphic to the division ring of real Quaternions. We let $\operatorname{Sym}(G)$ denote the subset of $\operatorname{Irr}(G)$ consisting of those characters that are symplectic. Then, if $E$ is either $\mathbb{Q}$ or $\mathbb{R}$, the decomposition (4) combines with the HasseSchilling-Maass Norm Theorem to imply that

$$
\operatorname{im}\left(\operatorname{Nrd}_{E[G]}\right)=\zeta(E[G])^{\times} \cap\left(\prod_{\kappa \in \operatorname{Sym}(G)} \mathbb{R}_{>0}^{\times} \times \prod_{\kappa \in \operatorname{Irr}(G) \backslash \operatorname{Sym}(G)}\left(E_{\kappa} \mathbb{R}\right)^{\times}\right)
$$

where $\mathbb{R}_{>0}^{\times}$is the multiplicative group of strictly positive real numbers (cf. [37, $(7.48)])$.

For typographic simplicity, in this section we shall usually omit explicit reference to the finitely generated ring $\Lambda$ which occurs in Theorem 5.1.1, preferring instead to write $\mathcal{O}_{E}$ in place of $\mathcal{O}_{E, \Lambda}$ and $\otimes$ in place of $\otimes_{\Lambda}$ etc.

6.1. The proof of Theorem 5.1.1(i). The existence of the isomorphism $\lambda_{\tau}$ implies that the $\mathbb{Q}[G]$-modules $\mathbb{Q} \otimes H_{\tau}^{0}$ and $\mathbb{Q} \otimes H_{\tau}^{1}$ are isomorphic (cf. the proof of Lemma 3.2.1(ii)). We may therefore fix a $G$-module homomorphism $\varphi: H_{\tau}^{1} \rightarrow H_{\tau}^{0}$ for which $\mathbb{Q} \otimes \varphi$ is bijective.

For each $\psi \in \operatorname{Irr}(G)$ we define $A_{\varphi}^{\tau}(\psi) \in \mathbb{C}^{\times}$as in Theorem 5.1.1(i).

Lemma 6.1.1. The element $\chi(\tau)$ belongs to $K_{0}(\Lambda[G], \mathbb{Q}[G])$ if and only if for every $\psi \in \operatorname{Irr}(G)$ and $\omega \in \operatorname{Aut}_{\mathbb{Q}}(\mathbb{C})$ one has

$$
A_{\varphi}^{\tau}(\omega \circ \psi)=\omega\left(A_{\varphi}^{\tau}(\psi)\right) .
$$


Proof. In view of the explicit description of $\operatorname{im}\left(\operatorname{Nrd}_{\mathbb{R}}[G]\right)$ given by (13), the Weak Approximation Theorem guarantees the existence of an element $\xi$ of $\zeta(\mathbb{Q}[G])^{\times}$ with

$$
\xi \mathcal{L}_{\tau}^{*} \in \operatorname{im}\left(\operatorname{Nrd}_{\mathbb{R}[G]}\right)
$$

We fix such an element $\xi$, set $x:=\xi \mathcal{L}_{\tau}^{*}$ and define $y=\left(y_{\psi}\right)_{\psi}$ in $\zeta(\mathbb{C}[G])^{\times}$by setting

$$
y_{\psi}:=\operatorname{det}_{\mathbb{C}}\left(\left(\mathbb{C} \otimes_{\mathbb{R}} \lambda_{\tau}\right) \circ(\mathbb{C} \otimes \varphi) \mid \operatorname{Hom}_{\mathbb{C}[G]}\left(V_{\psi}, \mathbb{C} \otimes H_{\tau}^{1}\right)\right)
$$

for every $\psi \in \operatorname{Irr}(G)$. Then $\delta\left(\mathcal{L}_{\tau}^{*}\right)-\delta(x)=-\delta(\xi)$ belongs to $K_{0}(\mathbb{Z}[G], \mathbb{Q}[G])$. Also $y=\operatorname{Nrd}_{\mathbb{R}[G]}(\hat{y})$ where $\hat{y} \in K_{1}(\mathbb{R}[G])$ corresponds to the automorphism $\lambda_{\tau} \circ(\mathbb{R} \otimes \varphi)$ of $\mathbb{R} \otimes H_{\tau}^{1}$ and so [16, Prop. 1.2.1(iv)] implies that $\left[\epsilon_{\tau}, \lambda_{\tau}\right]-\delta(y)=$ $\left[\epsilon_{\tau}, \varphi^{-1}\right] \in K_{0}(\Lambda[G], \mathbb{Q}[G])$. Hence one has $\chi(\tau) \in K_{0}(\Lambda[G], \mathbb{Q}[G])$ if and only if $\delta\left(y x^{-1}\right) \in K_{0}(\Lambda[G], \mathbb{Q}[G])$. Now $\delta\left(y x^{-1}\right)$ belongs to $K_{0}(\Lambda[G], \mathbb{Q}[G])$ if and only if $\xi y x^{-1}$ belongs to $\zeta(\mathbb{Q}[G])=H^{0}\left(\operatorname{Aut}_{\mathbb{Q}}(\mathbb{C}), \zeta(\mathbb{C}[G])\right)$. But for each $\psi \in \operatorname{Irr}(G)$ one has $\left(\xi y x^{-1}\right)_{\psi}=A_{\varphi}^{\tau}(\psi)$ and so the $\operatorname{Aut}_{\mathbb{Q}}(\mathbb{C})$-invariance of $\xi y x^{-1}$ is thus equivalent to the validity of the equalities (14), as claimed.

6.2. The proof of Theorem 5.1.1(ii). We assume henceforth that $\chi(\tau)$ belongs to $K_{0}(\Lambda[G], \mathbb{Q}[G])$. We fix a field $E$ and lattice $T_{\psi}$ as in Theorem 5.1.1(ii) and set $\mathcal{O}:=\mathcal{O}_{E}$. Then Lemma 6.1.1 implies that $A_{\varphi}^{\tau}(\psi)$ belongs to $E^{\times}$and we now investigate the fractional $\mathcal{O}$-ideal that is generated by this element.

For each $\psi \in \operatorname{Irr}(G)$ we define a left, resp. right exact functor on $\Lambda[G]$-modules $X$ by setting

$$
\begin{aligned}
X^{\psi} & :=\operatorname{Hom}_{\mathcal{O}}\left(T_{\psi}, \mathcal{O} \otimes X\right)^{G}, \\
X_{\psi} & :=\operatorname{Hom}_{\mathcal{O}}\left(T_{\psi}, \mathcal{O} \otimes X\right)_{G}
\end{aligned}
$$

(where the Hom-sets are endowed with the natural diagonal $G$-action). For each map of $\Lambda[G]$-modules $f: X \rightarrow Y$ we write $f_{\psi}$ and $f^{\psi}$ for the induced maps of $\mathcal{O}$-modules $X_{\psi} \rightarrow Y_{\psi}$ and $X^{\psi} \rightarrow Y^{\psi}$ respectively. There is a natural map of $\mathcal{O}$-modules

$$
X_{\psi} \stackrel{t(X, \psi)}{\longrightarrow} X^{\psi}
$$

that is induced by multiplication with $\sum_{g \in G} g$ on $\operatorname{Hom}_{\mathcal{O}}\left(T_{\psi}, \mathcal{O} \otimes X\right)$ and this map is bijective if $X$ is perfect because $\operatorname{Hom}_{\mathcal{O}}\left(T_{\psi}, \mathcal{O} \otimes X\right)$ is then a cohomologicallytrivial $G$-module. 
As in Theorem 5.1.1(ii), for each $\psi \in \operatorname{Irr}(G)$ we define $\varphi^{(\psi)}$ to be the composite homomorphism

$$
H_{\tau, \psi}^{1} \stackrel{t\left(H_{\tau}^{1}, \psi\right)}{\longrightarrow} H_{\tau}^{1, \psi} \stackrel{\varphi^{\psi}}{\longrightarrow} H_{\tau}^{0, \psi}
$$

and then define an Euler characteristic ideal by setting

$$
q_{\varphi}^{\tau}(\psi):=\operatorname{char}_{\mathcal{O}}\left(\operatorname{cok}\left(\varphi^{(\psi)}\right)\right) \operatorname{char}_{\mathcal{O}}\left(\operatorname{ker}\left(\varphi^{(\psi)}\right)\right)^{-1} .
$$

Proposition 6.2.1. If $\chi(\tau)$ belongs to $K_{0}(\Lambda[G], \mathbb{Q}[G])$, then it has finite order if and only if for each $\psi \in \operatorname{Irr}(G)$ one has

$$
A_{\varphi}^{\tau}(\psi) \mathcal{O}=q_{\varphi}^{\tau}(\psi)
$$

Proof. By replacing $E$ by a larger field if necessary, we may (and will) assume henceforth that for every $\psi \in \operatorname{Irr}(G)$ the lattice $T_{\psi}$ is a free $\mathcal{O}$-module.

We write $\mathfrak{M}$ for the maximal $\mathcal{O}$-order $\prod_{\psi \in \operatorname{Irr}(G)} \operatorname{End}_{\mathcal{O}}\left(T_{\psi}\right)$ in the $E$-algebra $\prod_{\psi \in \operatorname{Irr}(G)} \operatorname{End}_{E}\left(E \otimes_{\mathcal{O}} T_{\psi}\right) \cong E[G]$. Setting $T_{\psi}^{*}:=\operatorname{Hom}_{\mathcal{O}}\left(T_{\psi}, \mathcal{O}\right)$, the theory of Morita equivalence implies that the functor $\left(\prod_{\psi \in \operatorname{Irr}(G)} T_{\psi}^{*}\right) \otimes_{\mathfrak{M}}-$ induces a group isomorphism $K_{0}(\mathfrak{M}, E[G]) \stackrel{\sim}{\longrightarrow} \bigoplus_{\operatorname{Irr}(G)} K_{0}(\mathcal{O}, E)$ (cf. [23, (37)]). In addition, for each perfect $\mathbb{Z}[G]$-module $X$ one has a canonical isomorphism of $\mathcal{O}$-modules

$$
T_{\psi}^{*} \otimes_{\mathfrak{M}}\left(\mathfrak{M} \otimes_{\mathcal{O}[G]}(\mathcal{O} \otimes X)\right) \stackrel{\sim}{\longrightarrow} X^{\psi}
$$

and so $\left[23\right.$, Lem. 11d)] implies that an element of $K_{0}(\Lambda[G], \mathbb{Q}[G])$ has finite order if and only if for every $\psi \in \operatorname{Irr}(G)$ it belongs to the kernel of the homomorphism

$$
\rho_{*}^{\psi}: K_{0}(\Lambda[G], \mathbb{C}[G]) \rightarrow K_{0}(\mathcal{O}, \mathbb{C})
$$

that is induced by the functor $X \mapsto X^{\psi}$. It therefore suffices for us to prove that equality (17) is equivalent to the containment $\chi(\tau) \in \operatorname{ker}\left(\rho_{*}^{\psi}\right)$.

To do this we set $\lambda:=\lambda_{\tau}$ and $\epsilon:=\epsilon_{\tau}$, fix a representative of $\epsilon$ of the form $\left(\Psi^{\bullet}, \mathrm{id}, \mathrm{id}\right)$ where $\Psi^{i}$ is finitely generated and of finite projective dimension for $i \in\{0,1\}$ and $\Psi^{i}=0$ for $i \notin\{0,1\}$, and we set $H^{i}:=H_{\tau}^{i}$ and $t_{\psi}^{i}:=t\left(\Psi^{i}, \psi\right)$ for $i \in\{0,1\}$. Then the tautological exact sequence

$$
0 \rightarrow H^{0} \stackrel{\iota}{\rightarrow} \Psi^{0} \stackrel{d}{\rightarrow} \Psi^{1} \stackrel{\pi}{\rightarrow} H^{1} \rightarrow 0
$$


induces a commutative diagram of $\mathcal{O}$-modules with exact rows

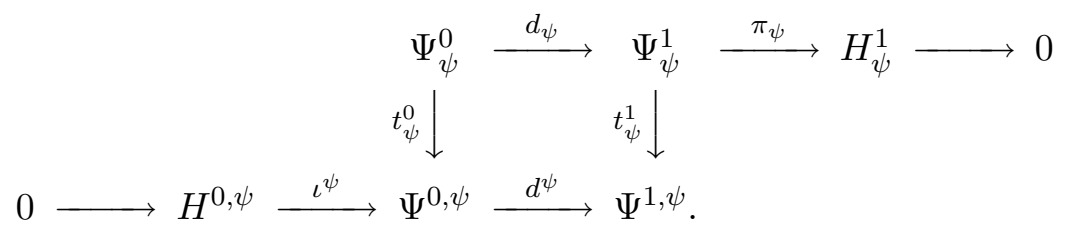

Both vertical morphisms here are bijective since the $\Lambda[G]$-modules $\Psi^{i}$ are perfect and so the diagram gives rise to an exact sequence of $\mathcal{O}$-modules

$$
0 \rightarrow H^{0, \psi} \stackrel{\iota^{\psi}}{\longrightarrow} \Psi^{0, \psi} \stackrel{d^{\psi}}{\longrightarrow} \Psi^{1, \psi} \stackrel{\pi_{\psi} \circ\left(t_{\psi}^{1}\right)^{-1}}{\longrightarrow} H_{\psi}^{1} \rightarrow 0
$$

Now $\mathcal{O}$ is a Dedekind domain and so any finitely generated $\mathcal{O}$-module $N$ is perfect and hence gives rise to an associated object $[N]$ of the category $V(\mathcal{O})$. If $X$ and $Y$ are any finitely generated $\mathcal{O}$-modules and $\mu: \mathbb{C} \otimes_{\mathcal{O}} X \rightarrow \mathbb{C} \otimes_{\mathcal{O}} Y$ is any isomorphism of $\mathbb{C}$-modules, then we set

$$
\operatorname{cl}_{\mathcal{O}}(X, Y ; \mu):=\left([X][Y]^{-1}, \mu_{\text {triv }}\right) \in K_{0}(\mathcal{O}, \mathbb{C})
$$

where $\mu_{\text {triv }}$ is the morphism $\left[\mathbb{C} \otimes_{\mathcal{O}} X\right]\left[\mathbb{C} \otimes_{\mathcal{O}} Y\right]^{-1} \rightarrow \mathbf{1}_{\mathbb{C}}$ induced by $\mu$.

Hence, if we set $t^{\psi}:=\mathbb{C} \otimes_{\mathcal{O}} t\left(H_{\tau}^{1}, \psi\right)$, then the exact sequence (19) implies

$$
\begin{aligned}
\rho_{*}^{\psi}\left(\left[\epsilon_{\tau}, \lambda\right]\right)=\left[\Psi^{\psi, \bullet}, \lambda^{\psi}\right]=\operatorname{cl}_{\mathcal{O}}\left(H^{0, \psi}, H_{\psi}^{1} ; t^{\psi,-1} \circ \lambda^{\psi}\right) \\
=-\operatorname{cl}_{\mathcal{O}}\left(H_{\psi}^{0}, H^{0, \psi} ; t^{\psi}\right)+\operatorname{cl}_{\mathcal{O}}\left(H_{\psi}^{0}, H_{\psi}^{1} ; \lambda^{\psi}\right) .
\end{aligned}
$$

Here $\Psi^{\psi, \bullet}$ is the complex $\Psi^{0, \psi} \stackrel{d^{\psi}}{\longrightarrow} \Psi^{1, \psi}$ where the first term occurs in degree 0 and the cohomology is identified with $H^{0, \psi}$ and $H_{\psi}^{1}$ via the maps in (19), and the third equality follows from the defining relations of $K_{0}(\mathcal{O}, \mathbb{C})$.

To deduce the claimed result from (20) we use the natural isomorphism $\iota: I_{\mathcal{O}} \cong$ $K_{0}(\mathcal{O}, \mathbb{C})$ where $I_{\mathcal{O}}$ denotes the multiplicative group of invertible $\mathcal{O}$-modules in $\mathbb{C}$. (This isomorphism is induced by the exact sequence (2) with $\mathfrak{A}=\mathcal{O}$ and $E=\mathbb{C}$ together with the canonical isomorphisms $K_{1}(\mathbb{C}) \stackrel{\sim}{\longrightarrow} \mathbb{C}^{\times}$and $K_{1}(\mathcal{O}) \stackrel{\sim}{\longrightarrow}$ $\left.\mathcal{O}^{\times}\right)$. Indeed, one has $\iota\left(q_{\varphi}^{\tau}(\psi)\right)=\operatorname{cl}_{\mathcal{O}}\left(H_{\psi}^{1}, H_{\psi}^{0} ; \mathbb{C} \otimes_{\mathcal{O}} \varphi_{\psi}\right)+\operatorname{cl}_{\mathcal{O}}\left(H_{\psi}^{0}, H^{0, \psi} ; t^{\psi}\right)$ and 
$\iota\left(\mathcal{O} \cdot \mathcal{L}_{\tau, \psi}^{*}\right)=\rho_{*}^{\psi}\left(\mathcal{L}_{\tau}^{*}\right)$ and hence $(20)$ implies that

$$
\begin{aligned}
& \rho_{*}^{\psi}(\chi(\tau)) \\
= & \rho_{*}^{\psi}\left(\left[\epsilon_{\tau}, \lambda_{\tau}\right]\right)-\rho_{*}^{\psi}\left(\mathcal{L}_{\tau}^{*}\right) \\
= & \left(\iota\left(q_{\varphi}^{\tau}(\psi)\right)+\rho_{*}^{\psi}\left(\left[\epsilon_{\tau}, \lambda_{\tau}\right]\right)\right)-\iota\left(q_{\varphi}^{\tau}(\psi)\right)-\rho_{*}^{\psi}\left(\mathcal{L}_{\tau}^{*}\right) \\
= & \operatorname{cl}_{\mathcal{O}}\left(H_{\psi}^{1}, H_{\psi}^{1} ; \lambda^{\psi} \circ\left(\mathbb{C} \otimes_{\mathcal{O}} \varphi_{\psi}\right)\right)-\iota\left(q_{\varphi}^{\tau}(\psi)\right)-\iota\left(\mathcal{O} \cdot \mathcal{L}_{\tau, \psi}^{*}\right) \\
= & \iota\left(\left(\mathcal{L}_{\tau, \psi}^{*}\right)^{-1} \operatorname{det}_{\mathbb{C}}\left(\left(\mathbb{C} \otimes_{\mathbb{R}} \lambda\right) \circ(\mathbb{C} \otimes \varphi) \mid \operatorname{Hom}_{\mathbb{C}[G]}\left(V_{\psi}, \mathbb{C} \otimes H^{1}\right)\right) \cdot q_{\varphi}^{\tau}(\psi)^{-1}\right) \\
= & \iota\left(A_{\varphi}^{\tau}(\psi) \cdot q_{\varphi}^{\tau}(\psi)^{-1}\right) .
\end{aligned}
$$

Thus (17) is valid if and only if $\chi(\tau)$ belongs to $\operatorname{ker}\left(\rho_{*}^{\psi}\right)$, as required.

\section{Remark 6.2.2. Maximal orders}

If $\mathfrak{M}$ is any maximal $\Lambda$-order in $\mathbb{Q}[G]$ with $\Lambda[G] \subseteq \mathfrak{M}$, then $K_{0}(\Lambda[G], \mathbb{Q}[G])_{\text {tor }}$ is the kernel of the natural homomorphism $K_{0}(\Lambda[G], \mathbb{Q}[G]) \rightarrow K_{0}(\mathfrak{M}, \mathbb{Q}[G])[24$, Lem. 11d)] and $T \Omega\left(M_{K}, \mathfrak{M}\right)$ is the image of $T \Omega\left(M_{K}, \Lambda[G]\right)$ under this homomorphism [24, Th. 4.1]. From (8), (9), Lemma 11.1.2 and (60) it therefore follows that, in the context of $\S 4$, the relations described in Theorem 5.1.1(i) and (ii) are consequences of $\operatorname{ETNC}\left(M_{K}, \mathfrak{M}\right)$.

Remark 6.2.3. Degree zero components

If $\psi$ is such that $\operatorname{Hom}_{\mathbb{C}[G]}\left(V_{\psi}, \mathbb{C} \otimes H_{\tau}^{1}\right)=0$, then (14) implies that $A_{\varphi}^{\tau}(\psi)^{-1}=$ $\mathcal{L}_{\tau, \psi}^{*}$ belongs to $E^{\times}$. In addition, since in this case both $H_{\tau}^{0, \psi}$ and $H_{\tau, \psi}^{1}$ are finite $\mathcal{O}$-modules, one has $q_{\varphi}^{\tau}(\psi)=\operatorname{char}_{\mathcal{O}}\left(H_{\tau}^{0, \psi}\right) \operatorname{char}_{\mathcal{O}}\left(H_{\tau, \psi}^{1}\right)^{-1}$ and hence (17) simplifies to give an equality of fractional $\mathcal{O}$-ideals

$$
\mathcal{L}_{\tau, \psi}^{*} \cdot \mathcal{O}=\operatorname{char}_{\mathcal{O}}\left(H_{\tau}^{0, \psi}\right)^{-1} \operatorname{char}_{\mathcal{O}}\left(H_{\tau, \psi}^{1}\right)
$$

\section{Remark 6.2.4. Tate motives}

- If $\tau=\tau_{0}$ as in Proposition 4.2.2, then it is clear that (14) is equivalent to the main conjecture of Stark at $s=0$ (as reformulated by Tate in [94, Chap. I, Conj. 5.1]) and that (17) is equivalent to the 'Strong-Stark Conjecture' formulated by Chinburg in [29, Conj. 2.2], and hence also to a special case of the conjecture formulated by Lichtenbaum in [71]. In recent work of Johnston and the present author [27] it has also been shown that in this case the equality (17) gives rise to a natural Stickelberger-type theorem for non-abelian extensions. 
- If $\tau=\tau_{1}$ as in Proposition 4.2.5, then [13, Prop. 3.6(i)] combines with (9) to show that (14) is equivalent to the main conjecture of Stark at $s=1$ (as reformulated by Tate in [94, Chap. I, Conj. 8.2]). However, in this context the (conjectural) equality (17) is new.

- If $\tau=\tau_{r}$ with $r<0$ as in Proposition 4.2.6, then (14) is easily shown to be equivalent to the main conjecture formulated by Gross in [56] (cf. also [33, Conj. 6.7]). In addition, in $\S 11$ we will show that (17) is in this case a refinement of the conjecture formulated by Chinburg, Kolster, Pappas and Snaith in [33, Conj. 6.12]. The final claim of Proposition 4.2.6 thus shows that [33, Conj. 6.12] is a consequence of $\operatorname{ETNC}\left(\mathbb{Q}(r)_{K}, \mathbb{Z}[G]\right)$. This observation answers the question posed by Chinburg et al [33, top of p. 357] of how their conjecture [33, Conj. $6.12]$ is related to Tamagawa number conjectures.

\section{Remark 6.2.5. Elliptic curves}

We assume the notation and hypotheses of $\S 4.3$ and fix a character $\psi \operatorname{in} \operatorname{Irr}(G)$. For each $\mathbb{Z}[G]$-module $N$ we write $N^{\psi}$ and $N_{\psi}$ in place of $\left(\Lambda \otimes_{\mathbb{Z}} N\right)^{\psi}$ and $\left(\Lambda \otimes_{\mathbb{Z}} N\right)_{\psi}$ respectively.

- The rank zero case. Assume first that $L(E, \bar{\psi}, 1) \neq 0$. Then [23, Conj. 4(ii)] for the pair $\left(h^{1}\left(E_{/ K}\right)(1), \mathbb{Q}[G]\right)$ implies that $E(K)^{\psi}$ (and hence also $\operatorname{Sel}\left(E_{/ K}\right)_{\psi}$ ) is finite. Thus $E(K)^{\psi}=\left(E(K)_{\text {tor }}\right)^{\psi}$ and so if $\mathfrak{p}$ is any prime ideal of $\mathcal{O}$ which does not divide the order of $E(K)_{\text {tor }}$, then $\mathcal{O}_{\mathfrak{p}} \otimes_{\mathcal{O}} E(K)^{\psi}$ vanishes. Hence Remark 6.2.3 combines with Proposition 4.3.1 to imply that if [23, Conj. 4(ii)] is valid for the pair $\left(h^{1}\left(E_{/ K}\right)(1), \mathbb{Q}[G]\right)$, if $\operatorname{ETNC}\left(h^{1}\left(E_{/ K}\right)(1), \Lambda[G]\right)$ is valid and if $\amalg\left(E_{/ K}\right)$ is finite, then $\operatorname{Sel}\left(E_{/ K}\right)_{\psi}$ is finite and for all prime ideals $\mathfrak{p}$ as above one has

$$
\operatorname{val}_{\mathfrak{p}}\left(\Omega(E)^{-\psi(1)} \tau^{*}(\psi) L_{S_{\mathrm{ram}}}(E, \bar{\psi}, 1)\right)=\operatorname{length}_{\mathcal{O}_{\mathfrak{p}}}\left(\mathcal{O}_{\mathfrak{p}} \otimes_{\mathcal{O}} \operatorname{Sel}\left(E_{/ K}\right)_{\psi}\right)
$$

This explicit formula is in the same spirit as the predictions made by Coates, Fukaya, Kato, Sujatha and Venjakob in [35, Cor. 5.10]. In particular, it should be possible to obtain numerical evidence in support of the above formula by using the kind of techniques developed by Dokchister and Dokchister in [40].

- The higher rank case. If $L(E, \bar{\psi}, 1)=0$, then the statements of Theorem 5.1.1(i) and (ii) with $\tau=\tau_{E, \Lambda}$ can be interpreted as asserting the existence of elements of $\Lambda \otimes_{\mathbb{Z}} E(K)$ that are related (via the Néron-Tate height pairing) in a precise way to the value at $s=1$ of an appropriate derivative of $L(E, \bar{\psi}, s)$. 
Numerical evidence in favour of such predictions has recently been obtained by Fearnley and Kisilevsky [43] and Bley [7].

\section{Explicit MOdule STRUCTURES}

In this section we prove both Theorem 5.1.1(iii) and Theorem 5.2.1 and also discuss several explicit arithmetic applications of these results.

7.1. The general case. In this section we prove Theorem 5.1.1(iii). To this end we recall that the image of the connecting homomorphism $\partial_{\Lambda}^{0}:=\partial_{\Lambda[G], \mathbb{R}}^{0}$ in diagram (2) is equal to the locally-free class group $\mathrm{Cl}(\Lambda[G])$ of $\Lambda[G]$ (as discussed, for example, in $[37, \S 49])$. We also set

$$
\chi\left(\epsilon_{\tau}\right):=\partial_{\Lambda}^{0}\left(\left[\epsilon_{\tau}, \lambda_{\tau}\right]\right)
$$

and

$$
w(\tau):=\partial_{\Lambda}^{0}\left(\delta_{\Lambda}\left(\mathcal{L}_{\tau}^{*}\right)\right)
$$

We recall (from $\S 2.6$ ) that each finitely generated cohomologically-trivial $\Lambda[G]$ module $N$ gives rise (via a choice of projective resolution) to an associated element $(N)$ of $K_{0}(\Lambda[G])$. In particular, one has

$$
\chi\left(\epsilon_{\tau}\right)=\left(\Psi^{0}\right)-\left(\Psi^{1}\right)
$$

where $\Psi^{0}$ and $\Psi^{1}$ are any finitely generated cohomologically-trivial $\Lambda[G]$-modules lying in any representative (18) of the class $\epsilon_{\tau}$.

Before stating the next result we note that, in terms of the description (13), one knows that $\mathcal{L}_{\tau, \kappa}^{*}$ belongs to $\mathbb{R}^{\times}$for each $\kappa$ in $\operatorname{Sym}(G)\left(\right.$ as $\left.\mathcal{L}_{\tau}^{*} \in \zeta(\mathbb{R}[G])^{\times}\right)$.

Proposition 7.1.1. If $\chi(\tau)$ belongs to $\operatorname{ker}\left(\partial_{\Lambda}^{0}\right)$, then all of the following claims are valid.

(i) $\chi\left(\epsilon_{\tau}\right)$ depends only upon the sign of $\mathcal{L}_{\tau, \kappa}^{*}$ for each $\kappa$ in $\operatorname{Sym}(G)$. In particular, if $\mathcal{L}_{\tau, \kappa}^{*}$ is strictly positive for each such $\kappa$, then $\chi\left(\epsilon_{\tau}\right)=0$.

(ii) If $\operatorname{Sym}(G)$ is empty, then $\chi\left(\epsilon_{\tau}\right)=0$.

(iii) $2 \chi\left(\epsilon_{\tau}\right)=0$.

(iv) $\left(\psi^{*}-1\right) \chi\left(\epsilon_{\tau}\right)=0$ with $\psi^{*}$ the involution of $K_{0}(\Lambda[G])$ defined in $\S 2.5$. 
Proof. It is clear that $\chi(\tau)$ belongs to $\operatorname{ker}\left(\partial_{\Lambda}^{0}\right)$ if and only if

$$
\chi\left(\epsilon_{\tau}\right)=w(\tau) \in \operatorname{Cl}(\Lambda[G]) .
$$

To prove claim (i) it therefore suffices to prove that $w(\tau)$ is determined by the signs of $\mathcal{L}_{\tau, \kappa}^{*}$ for each $\kappa$ in $\operatorname{Sym}(G)$ (and is trivial if none of these numbers $\mathcal{L}_{\tau, \kappa}^{*}$ is negative). To show this we choose $\xi \in \zeta(\mathbb{Q}[G])^{\times}$satisfying (15). Then $\delta_{\Lambda}\left(\xi \mathcal{L}_{\tau}^{*}\right)=\partial_{\Lambda[G], \mathbb{R}}^{1}\left(\operatorname{Nrd}_{\mathbb{R}[G]}^{-1}\left(\xi \mathcal{L}_{\tau}^{*}\right)\right) \in \operatorname{im}\left(\partial_{\Lambda[G], \mathbb{R}}^{1}\right)=\operatorname{ker}\left(\partial_{\Lambda}^{0}\right)$ and so the explicit description of $\delta_{\Lambda}$ in $[23, \S 4.2]$ implies that $w(\tau)=-\delta_{\Lambda}(\xi)=-\sum_{p \in \operatorname{Spec}(\Lambda)} \delta_{p}(\xi)$ where, for each such $p$, we write $\delta_{p}$ for the natural composite homomorphism $\zeta(\mathbb{Q}[G])^{\times} \hookrightarrow \zeta\left(\mathbb{Q}_{p}[G]\right)^{\times} \cong K_{1}\left(\mathbb{Q}_{p}[G]\right) \rightarrow K_{0}\left(\mathbb{Z}_{p}[G], \mathbb{Q}_{p}[G]\right) \subset K_{0}(\Lambda[G], \mathbb{Q}[G])$, where the isomorphism is given by the bijective reduced norm map $\operatorname{Nrd}_{\mathbb{Q}_{p}[G]}$.

We next recall the idelic descriptions of $K_{0}(\Lambda[G], \mathbb{Q}[G])$ and $\operatorname{Cl}(\Lambda[G])$. To do so, we write $J_{f}(\zeta(\mathbb{Q}[G]))$ for the group of elements $\left(u_{p}\right)_{p} \in \prod_{p} \zeta\left(\mathbb{Q}_{p}[G]\right)^{\times}$ (product over all primes $p$ in $\operatorname{Spec}(\Lambda)$ ) such that $u_{p} \in \zeta\left(\mathbb{Z}_{p}[G]\right)^{\times}$for almost all $p$. We regard $\zeta(\mathbb{Q}[G])^{\times}$as embedded diagonally inside $J_{f}(\zeta(\mathbb{Q}[G]))$ and let $\operatorname{Nrd}\left(U_{f}(\Lambda[G])\right)$ denote the subgroup of $J_{f}(\zeta(\mathbb{Q}[G]))$ consisting of those elements $\left(u_{p}\right)_{p}$ with $u_{p} \in \operatorname{Nrd}_{\mathbb{Q}_{p}[G]}\left(\operatorname{im}\left(K_{1}\left(\mathbb{Z}_{p}[G]\right) \rightarrow K_{1}\left(\mathbb{Q}_{p}[G]\right)\right)\right)$ for all primes $p \in \operatorname{Spec}(\Lambda)$. Then there is a commutative diagram

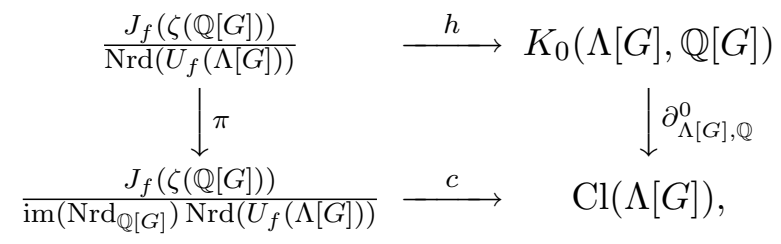

where $\pi$ is the natural projection map and $h$ and $c$ are the isomorphisms described in $[37,49.17]$. In terms of this diagram one has $\sum_{p} \delta_{p}(\xi)=h(\xi)$ and so $w(\tau)=$ $-c(\xi)$.

The equality $(13)$ (with $E=\mathbb{Q}$ ) makes it clear that the class $c(\xi)$ depends only upon the sign of the real number $\xi_{\kappa}$ for each symplectic character $\kappa \in$ $\operatorname{Sym}(G)$, and in conjunction with (15) further implies that, for each such $\kappa$, one has $\xi_{\kappa} \mathcal{L}_{\tau, \kappa}^{*}>0$. This proves both claims (i) and (ii).

In a similar way, claim (iii) is a consequence of the fact that the square of any element of $\zeta(\mathbb{Q}[G])^{\times}$belongs to $\operatorname{ker}\left(\partial_{\Lambda}^{0} \circ \delta_{\Lambda}\right)$.

Finally we note that if $\xi$ is any element of $\zeta(\mathbb{Q}[G])^{\times}$, then $\psi^{*}(c(\xi))=-c\left(\xi^{\#}\right)$ (see $\S 2.5$ ) and so claim (iv) simply follows from the fact that $\left(\xi^{\#}\right)_{\kappa}=\xi_{\bar{\kappa}}=\xi_{\kappa}$ for each $\kappa$ in $\operatorname{Sym}(G)$. 


\section{Remark 7.1.2. Explicit structural restrictions}

Claims (ii) and (iii) of Proposition 7.1.1 combine with locally-free cancellation to constitute strong restrictions on the structures of the $\Lambda[G]$-modules $\Psi^{0}$ and $\Psi^{1}$ in (21). For example, if we assume (as we may) that $\Psi^{1}$ is a finitely generated free $\Lambda[G]$-module and choose a resolution of $\Psi^{0}$ of the form $0 \rightarrow P \rightarrow F \rightarrow \Psi^{0} \rightarrow 0$ where $F$ is a finitely generated free $\Lambda[G]$-module, then the relation described in claim (ii), resp. (iii), of Proposition 7.1 .1 would imply that the $\Lambda[G]$-module $P$, resp. $P \oplus P$, is free.

\section{Remark 7.1.3. Tate motives}

We assume the notation of $\S 4.2$. Then $L_{K / k, S}^{*}(t)$ belongs to $\operatorname{im}\left(\operatorname{Nrd}_{\mathbb{R}[G]}\right)$ for each strictly positive integer $t$ and hence, for each integer $t \leq 0$, the functional equation of Artin $L$-functions implies that the question of whether $L_{K / k, S}^{*}(t)$ belongs to $\operatorname{im}\left(\operatorname{Nrd}_{\mathbb{R}[G]}\right)$ is determined by the signs of epsilon constants at elements of $\operatorname{Sym}(G)$ (this was first observed by Chinburg in [29]). This fact combines with the argument of Proposition 7.1.1(i) to show that $w\left(\tau_{r}\right)$ is equal to 0, resp. to the Cassou-Noguès-Fröhlich root number class $w(K / k)$ defined in [31, p. 358], if $r \geq 1$, resp. $r \leq 0$.

- If $S$ is large enough to ensure $\operatorname{Pic}\left(\mathcal{O}_{K, S}\right)$ vanishes, then [22, Prop. 3.5] implies that $\chi\left(\epsilon_{\tau_{0}}\right)$ is equal to the element $\Omega(K / k, 3)$ defined by Chinburg in $[29,31]$. Thus $\chi\left(\tau_{0}\right)$ belongs to $\operatorname{ker}\left(\partial_{\mathbb{Z}}^{0}\right)$ if and only if the ' $\Omega_{3}$-Conjecture' of loc. cit. is valid for $K / k$.

- From (9) and [13, the proof of Prop. 3.6(ii)] one has $\chi\left(\epsilon_{\tau_{1}}\right)=\psi^{*}(\Omega(K / k, 1))$ where $\Omega(K / k, 1)$ is the element defined by Chinburg in [31]. Thus $\chi\left(\tau_{1}\right)$ belongs to $\operatorname{ker}\left(\partial_{\mathbb{Z}}^{0}\right)$ if and only if the ' $\Omega_{1}$-Conjecture' of loc. cit. is valid for $K / k$.

- If $r<0$, then $\chi\left(\epsilon_{\tau_{r}}\right)$ is equal to the element $\Omega_{-r}(K / k)$ defined by Chinburg et al in [33] (see $\S 11$ for a proof of this fact). Thus $\chi\left(\tau_{r}\right)$ belongs to $\operatorname{ker}\left(\partial_{\mathbb{Z}}^{0}\right)$ if and only if the conjectural equality $\Omega_{-r}(K / k)=w(K / k)$ discussed in [33, Question 1.3] and [34] is valid.

Remark 7.1.4. Classical Galois module theory

Set $\chi^{\text {loc }}\left(\epsilon_{\tau_{1}}\right):=\psi^{*}\left(\left[\epsilon_{\tau_{1}}, \lambda_{\tau_{1}}\right]\right)+\left[\epsilon_{\tau_{0}}, \lambda_{\tau_{0}}\right]$. Recall also that if $K / k$ is at most tamely ramified, then the $\mathbb{Z}[G]$-module $\mathcal{O}_{K}$ is projective (by Noether's Theorem). Now the descriptions of $\chi\left(\epsilon_{\tau_{0}}\right)$ and $\chi\left(\epsilon_{\tau_{1}}\right)$ in Remark 7.1.3 combine with results of Chinburg in [31] to imply that if $K / k$ is at most tamely ramified, then the element 
$\partial_{\mathbb{Z}}^{0}\left(\chi^{\text {loc }}\left(\epsilon_{\tau_{1}}\right)\right)=\psi^{*}\left(\chi\left(\epsilon_{\tau_{1}}\right)\right)+\chi\left(\epsilon_{\tau_{0}}\right)$ is equal to $U_{K / k}:=\left(\mathcal{O}_{K}\right)-[k: \mathbb{Q}] \cdot(\mathbb{Z}[G])$. In this special case therefore, the predictions obtained by combining Propositions 4.2.2, 4.2.5 and 7.1.1 (for $\tau=\tau_{0}$ and $\tau=\tau_{1}$ ) recover the result that $U_{K / k}=0$ if $\operatorname{Sym}(G)=\emptyset$, resp. that $2 \cdot U_{K / k}=0$, resp. that $U_{K / k}=\psi^{*}\left(U_{K / k}\right)$. The last three results are due to $\mathrm{M}$. Taylor and are amongst the central results of classical Galois module theory (for more details see, for example, [49, Chap. I, §6]). The element $\chi^{\text {loc }}\left(\epsilon_{\tau_{1}}\right)$ is also itself of some independent interest and there is by now an extensive theory related to it (cf. $[1,8,10,87])$.

Remark 7.1.5. Elliptic curves

Using the notation and hypotheses of Proposition 4.3 .1 we set $\tau:=\tau_{E, \Lambda}$. We assume that $\operatorname{Sym}(G)$ is empty (as is automatically satisfied if, for example, $|G|$ is odd) so that Proposition 7.1.1(ii) shows $\operatorname{ETNC}\left(h^{1}\left(E_{/ K}\right)(1), \Lambda[G]\right)$ implies that $\chi\left(\epsilon_{\tau}\right)$ vanishes. If now $\ell$ is any prime in $\operatorname{Spec}(\Lambda)$ which does not divide the order of either $E(K)_{\text {tor }}, c_{E, \Lambda}^{0}(K)$ or $c_{E, \Lambda}^{1}(K)$, then the conjectural vanishing of $\chi\left(\epsilon_{\tau}\right)$ implies the existence of an exact sequence of finitely generated $\mathbb{Z}_{(\ell)}[G]$-modules of the form

$$
0 \rightarrow \mathbb{Z}_{(\ell)} \otimes E(K) \rightarrow F \rightarrow F \rightarrow \mathbb{Z}_{(\ell)} \otimes \operatorname{Sel}\left(E_{/ K}\right) \rightarrow 0,
$$

where $F$ is free. This shows, for example, that if $\operatorname{ETNC}\left(h^{1}\left(E_{/ K}\right)(1), \Lambda[G]\right)$ is valid and $\amalg\left(E_{/ K}\right)$ is finite, then for each such $\ell$ the $\mathbb{Z}_{(\ell)}[G]$-module $\mathbb{Z}_{(\ell)} \otimes \operatorname{Sel}\left(E_{/ K}\right)$ admits a presentation with the same number of generators and relations.

7.2. The case of $p$-groups. We assume throughout this section that $G$ is a group of $p$-power order for some prime $p$. We first prove Theorem 5.2.1 and then discuss explicit arithmetic applications of this result in the context of the examples described in $\S 4$.

We always abbreviate 'cohomologically-trivial' to 'c-t'.

7.2.1. The proof of Theorem 5.2.1. In this subsection we assume the notation and hypotheses of Theorem 5.2.1. Thus $G$ is cyclic and $p \in \operatorname{Spec}(\Lambda)$.

At the outset we note that for each subgroup $J$ of $G$ the Tate cohomology group $\hat{H}^{-1}\left(J, H^{1}\right)$ is a finite group of $p$-power order (which is trivial if $J$ is the trivial group) and hence identifies with a finite submodule of $\mathbb{Z}_{p} \otimes_{\Lambda} H_{J}^{1}$. Hence, our hypothesis that $\mathbb{Z}_{p} \otimes_{\Lambda} H_{J}^{1}$ is torsion-free for each non-trivial subgroup $J$ implies that $\hat{H}^{-1}\left(J, H^{1}\right)$ vanishes for all subgroups $J$. 
When considered in conjunction with the Tate cohomology of any sequence of the form (18) this fact implies (since the $G$-modules $\Psi^{0}$ and $\Psi^{1}$ in (18) are c-t) that $\hat{H}^{1}\left(J, H^{0}\right)=0$ for all $J$. But, since $J$ is cyclic, its Tate cohomology is periodic of order 2 and hence one also has $\hat{H}^{-1}\left(J, H^{0}\right)=0$. Finally, using the assumption that the $G$-module $H_{\text {tor }}^{0}$ is c-t, we deduce from the tautological exact sequence $0 \rightarrow H_{\mathrm{tor}}^{0} \rightarrow H^{0} \rightarrow H_{\mathrm{tf}}^{0} \rightarrow 0$ that $\hat{H}^{-1}\left(J, H_{\mathrm{tf}}^{0}\right)$ vanishes for each subgroup $J$ of $G$.

To prove Theorem 5.2.1(i) we need only therefore apply the following result with $N=\mathbb{Z}_{p} \otimes_{\Lambda} H_{\mathrm{tf}}^{0}$.

Proposition 7.2.1. Let $G$ be a cyclic group of p-power order and $N$ a finitely generated torsion-free $\mathbb{Z}_{p}[G]$-module. Then $N$ is a permutation lattice if and only if for every non-trivial subgroup $J$ of $G$ the group $\hat{H}^{-1}(J, N)$ vanishes.

Proof. This is a consequence of a more general result of Yakovlev in [97] and can also be proved directly [42].

At this stage we know that there is an isomorphism of $\mathbb{Z}_{p}[G]$-modules of the form

$$
\mathbb{Z}_{p} \otimes_{\Lambda} H_{\mathrm{tf}}^{0} \cong \bigoplus_{J \leq G} \mathbb{Z}_{p}[G / J]^{\left\langle n_{J}\right\rangle}
$$

for certain non-negative integers $n_{J}$.

To prove Theorem 5.2.1(ii) we write $\mathfrak{A}$ for the $\Lambda$-order of $\mathbb{Q}[G]$ that is generated over $\Lambda[G]$ by the idempotents $\left\{e_{J}: J \leq G\right.$ and $\left.H_{\mathrm{tf}}^{0}=\left(H_{\mathrm{tf}}^{0}\right)^{J}\right\}$ and $\mathfrak{M}$ for the (unique) maximal $\Lambda$-order in $\mathbb{Q}[G]$. We also note that, since $H_{\mathrm{tf}}^{0}$ is a finitely generated $\Lambda[G]$-module, the ring $\mathfrak{B}:=\operatorname{End}_{\mathbb{Q}[G]}\left(H_{\mathrm{tf}}^{0}\right)$ is a $\Lambda$-order in $\mathbb{Q}[G]$ that contains $\Lambda[G]$. Hence one has $\mathfrak{A} \subseteq \mathfrak{B} \subseteq \mathfrak{M}$ and so it suffices to prove that $\mathbb{Z}_{q} \otimes_{\Lambda} \mathfrak{B} \subseteq \mathbb{Z}_{q} \otimes_{\Lambda} \mathfrak{A}$ for all primes $q$ in $\operatorname{Spec}(\Lambda)$.

If $q \neq p$, then $\mathbb{Z}_{q} \otimes_{\Lambda} \mathfrak{A}=\mathbb{Z}_{q} \otimes_{\Lambda} \mathfrak{M}=\mathbb{Z}_{q}[G]$ and so there is nothing to prove. To deal with the case $q=p$ we use the fact that $\mathbb{Z}_{p} \otimes_{\Lambda} \mathfrak{M}$ is generated over $\mathbb{Z}_{p}[G]$ by the idempotents $\left\{e_{J}: J \leq G\right\}$ (cf. [5, $\S 2.2$, Lem. 2]). Indeed, with this description, it is straightforward to check that $\mathbb{Z}_{p} \otimes_{\Lambda} \mathfrak{A}$ is the maximal subring of $\mathbb{Z}_{p} \otimes_{\Lambda} \mathfrak{M}$ which preserves the right hand side of (23) (with respect to the natural multiplication action). Hence the isomorphism (23) implies that $\mathbb{Z}_{q} \otimes_{\Lambda} \mathfrak{B} \subseteq \mathbb{Z}_{q} \otimes_{\Lambda} \mathfrak{A}$, as required. 
Next we consider Theorem 5.2.1(iii). The isomorphism $\mathbb{C} \otimes_{\mathbb{R}} \lambda_{\tau}: \mathbb{C} \otimes_{\Lambda} H^{0} \cong$ $\mathbb{C} \otimes_{\Lambda} H^{1}$ of $\mathbb{C}[G]$-modules shows that the properties (iii)(a) and (iii)(b) are equivalent. Now $\operatorname{dim}_{\mathbb{C}}\left(e_{\chi}\left(\mathbb{C} \otimes_{\Lambda} H^{0}\right)\right)$ is independent of $\chi$ if and only if $\mathbb{C} \otimes_{\Lambda} H^{0}$ is a free $\mathbb{C}[G]$-module, or equivalently (by [37, Exer. 6, p. 139]) that $\mathbb{Q}_{q} \otimes_{\Lambda} H^{0}$ is a free $\mathbb{Q}_{q}[G]$-module for any (and therefore every) prime $q \in \operatorname{Spec}(\Lambda)$. This makes it clear both that (iii)(c) implies (iii)(a) and, given the isomorphism (23), that (iii)(a) is itself equivalent to asserting that $\mathbb{Z}_{p} \otimes_{\Lambda} H_{\mathrm{tf}}^{0}$ is a free $\mathbb{Z}_{p}[G]$-module. Also, if $\mathbb{Z}_{p} \otimes_{\Lambda} H_{\mathrm{tf}}^{0}$ is a free $\mathbb{Z}_{p}[G]$-module, then $\mathbb{Q} \otimes_{\Lambda} H_{\mathrm{tf}}^{0}$ is a free $\mathbb{Q}[G]$-module and hence $\mathbb{Q}_{q} \otimes_{\Lambda} H_{\mathrm{tf}}^{0}$ is a free $\mathbb{Q}_{q}[G]$-module for all primes $q \neq p$. But for any $q \neq p$ the ring $\mathbb{Z}_{q}[G]$ is regular so $\mathbb{Z}_{q} \otimes_{\Lambda} H_{\mathrm{tf}}^{0}$ is a projective $\mathbb{Z}_{q}[G]$-module and hence [37, Th. 32.1] implies that $\mathbb{Z}_{q} \otimes_{\Lambda} H_{\mathrm{tf}}^{0}$ is a free $\mathbb{Z}_{q}[G]$-module. Thus (iii)(a) implies (iii)(c).

Now property (iii)(c) implies that $H_{\mathrm{tf}}^{0}$ is c-t and hence, since $H_{\mathrm{tor}}^{0}$ is (by assumption) also c-t, that $H^{0}$ is itself c-t. On the other hand, the isomorphism (23) makes it clear that if the module $H^{0}$, and hence also $\mathbb{Z}_{p} \otimes_{\Lambda} H_{\mathrm{tf}}^{0}$, is c-t, then $\mathbb{Z}_{p} \otimes_{\Lambda} H_{\mathrm{tf}}^{0}$ is a free $\mathbb{Z}_{p}[G]$-module. Hence (iii)(c) is equivalent to (iii)(d).

Since the modules $\Psi^{0}$ and $\Psi^{1}$ in the exact sequence (18) are both c-t, it is also clear that $H^{0}$ is c-t precisely when $H^{1}$ is c-t and hence that properties (iii)(d) and (iii)(e) are equivalent.

Next we note that (iii)(c) implies (iii)(f). Indeed, if $H_{\mathrm{tf}}^{0}$ is a locally-free $\Lambda[G]$ module, then $\operatorname{End}_{\mathbb{Q}[G]}\left(H_{\mathrm{tf}}^{0}\right)=\Lambda[G]$ and so (iii)(f) is clear. To complete the proof of Theorem 5.2.1(iii) it is thus enough to prove that (iii)(f) implies (iii)(a). But if $H_{\mathrm{tf}}^{0}$ is a locally-free $\operatorname{End}_{\mathbb{Q}[G]}\left(H_{\mathrm{tf}}^{0}\right)$-module, then $\mathbb{C} \otimes_{\Lambda} H_{\mathrm{tf}}^{0}$ is a free $\mathbb{C}[G]$-module and so (iii)(a) is clear.

We now consider Theorem 5.2.1(iv). We therefore assume that the (equivalent) conditions of claim (iii) are valid. Then claim (iv)(a) follows directly upon combining Proposition 7.1.1(ii) with equality (21) and the fact that, since all modules in the exact sequence (18) are finitely generated and c-t, one has $\left(\Psi^{0}\right)-\left(\Psi^{1}\right)=\left(H^{0}\right)-\left(H^{1}\right)$ in $K_{0}(\Lambda[G])$.

If $\Lambda^{\prime}$ is as in (iv)(b), then (iv)(a) implies that $\left(\Lambda^{\prime} \otimes_{\Lambda} H_{\mathrm{tf}}^{0}\right)=\left(\Lambda^{\prime} \otimes_{\Lambda} H_{\mathrm{tf}}^{1}\right)$ in $K_{0}\left(\Lambda^{\prime}[G]\right)$. But (iii) implies that $\Lambda^{\prime} \otimes_{\Lambda} H_{\mathrm{tf}}^{0}$ and $\Lambda^{\prime} \otimes_{\Lambda} H_{\mathrm{tf}}^{1}$ are locally-free $\Lambda^{\prime}[G]$ modules (of the same rank). Hence, by locally-free cancellation [37, (49.3)], the $\Lambda^{\prime}[G]$-modules $\Lambda^{\prime} \otimes_{\Lambda} H^{0}=\Lambda^{\prime} \otimes_{\Lambda} H_{\mathrm{tf}}^{0}$ and $\Lambda^{\prime} \otimes_{\Lambda} H^{1}=\Lambda^{\prime} \otimes_{\Lambda} H_{\mathrm{tf}}^{1}$ are isomorphic. This proves claim (iv)(b). 
We defer the proof of Theorem 5.2.1(iv)(c) to $\S 8.3$.

7.2.2. Arithmetic applications. Before describing some arithmetic applications of Theorem 5.2.1 we record the following useful result.

Lemma 7.2.2. Let $G$ be a group of p-power order.

(i) For each intermediate field $F$ of $K / k$ we write $\tau_{F, 0}$ for the a.t.e. that is described in Proposition 4.2.2 with $K / k$ replaced by $F / k$. Then for each subgroup $J$ of $G$ there is a canonical isomorphism of $\mathbb{Z}_{p}[G / J]$-modules $\mathbb{Z}_{p} \otimes\left(H_{\tau_{K, 0}}^{1}\right)_{J} \cong \mathbb{Z}_{p} \otimes H_{\tau_{K^{J}, 0}}^{1}$.

(ii) The same assertions are valid if in claim (i) one replaces $\tau_{0}$ by either $\tau_{r}$ for a strictly negative integer $r$ (as in Proposition 4.2.6) or $\tau_{E, \Lambda}$ for an elliptic curve $E$ (as in Proposition 4.3.1), where in the latter case we use the fact that if the pair $(K / \mathbb{Q}, E)$ satisfies either condition $(A)$ or condition (B) of Proposition 4.3.1, then so does the pair $\left(K^{J} / \mathbb{Q}, E\right)$ for any subgroup $J$ of $G$.

Proof. For each subgroup $J$ we write $\Psi_{K^{J}, S}$ for the object of $D^{\text {perf }}(\mathbb{Z}[G / J])$ that occurs in the proof of Proposition 4.2.2 with $K / k$ replaced by $K^{J} / k$. Then there is a canonical 'projection formula' isomorphism $\mathbb{Z}[G / J] \otimes_{\mathbb{Z}[G]}^{\mathbb{L}} \Psi_{K, S} \cong \Psi_{K^{J}, S}$ in $D(\mathbb{Z}[G / J])$ (cf. [22, Lem. 11]). Also, since $H^{i}\left(\Psi_{K, S}\right)=0$ for all $i>1$, there is a canonical isomorphism of $G / J$-modules $H^{1}\left(\Psi_{K, S}\right)_{J} \cong H^{1}\left(\mathbb{Z}[G / J] \otimes_{\mathbb{Z}[G]}^{\mathbb{L}} \Psi_{K, S}\right)$. Claim (i) thus follows from the fact that the construction of Proposition 4.2.2 gives $H_{\tau_{K, 0}}^{1}=H^{1}\left(\Psi_{K, S}\right)$ and $H_{\tau_{K^{J}, 0}}^{1}=H^{1}\left(\Psi_{K^{J}, S}\right)$.

Claim (ii) is proved by a very similar argument. Indeed, if $\tau=\tau_{r}$ as in Proposition 4.2.6, then the same assertions as in claim (i) follow from the explicit construction of $\tau$ in $\S 11$ and the fact that for each prime $p$ there is a "projection formula' isomorphism in $D\left(\mathbb{Z}_{p}[G / J]\right)$ of the form

$$
\begin{aligned}
\mathbb{Z}_{p}[G / J] \otimes_{\mathbb{Z}_{p}[G]}^{\mathbb{L}} R \operatorname{Hom}_{\mathbb{Z}_{p}}\left(R \Gamma_{c}\left(\mathcal{O}_{K, S}\left[\frac{1}{p}\right], \mathbb{Z}_{p}(r)\right), \mathbb{Z}_{p}[-2]\right) \\
\cong R \operatorname{Hom}_{\mathbb{Z}_{p}}\left(R \Gamma_{c}\left(\mathcal{O}_{K^{J}, S}\left[\frac{1}{p}\right], \mathbb{Z}_{p}(r)\right), \mathbb{Z}_{p}[-2]\right) .
\end{aligned}
$$

Lastly, if $\tau=\tau_{E, \Lambda}$ is as in Proposition 4.3.1, then the required isomorphisms are a consequence of the explicit construction of $\tau$ at the end of $\S 12$ and the fact that for any subgroup $J$ of $G$ the definition of the complex $R \Gamma_{f}\left(\mathbb{Q}, \mathbb{Z}_{p}[G] \otimes_{\mathbb{Z}_{p}} T_{p}(E)\right)$ 
via (54) ensures that there is a natural isomorphism in $D\left(\mathbb{Z}_{p}[G / J]\right)$ of the form

$$
\mathbb{Z}_{p}[G / J] \otimes_{\mathbb{Z}_{p}[G]}^{\mathbb{L}} R \Gamma_{f}\left(\mathbb{Q}, \mathbb{Z}_{p}[G] \otimes_{\mathbb{Z}_{p}} T_{p}(E)\right) \cong R \Gamma_{f}\left(\mathbb{Q}, \mathbb{Z}_{p}[G / J] \otimes_{\mathbb{Z}_{p}} T_{p}(E)\right) .
$$

7.2.3. $\mathbb{Q}(0)_{K}$.

Proposition 7.2.3. Let $G$ be a group of p-power order and write $S$ for the (finite) set of places of $k$ comprising $S_{\infty}$ and those places which ramify in $K / k$.

(i) Assume $G$ is cyclic and that $p$ does not divide either $\left|k_{\text {tor }}^{\times}\right|$or $\left|\operatorname{Pic}\left(\mathcal{O}_{K^{J}, S}\right)\right|$ for any non-trivial subgroup $J$ of $G$. Then $\left(\mathcal{O}_{K, S}^{\times}\right)_{\mathrm{tf}}$ is a locally-free $\mathbb{Z}[G]$ module if and only if there exists a unique place of $k$ which ramifies in $K / k$ and the decomposition subgroup of this place is equal to $G$.

(ii) Assume that $p$ is odd and that there exists a unique place of $k$ which ramifies in $K / k$ and that the decomposition subgroup of this place is equal to $G$. If $p$ does not divide $\left|\operatorname{Pic}\left(\mathcal{O}_{k, S}\right)\right|$, then the $G$-modules $\mathcal{O}_{K, S}^{\times}$and $\operatorname{Pic}\left(\mathcal{O}_{K, S}\right)$ are both $c$-t. If $\operatorname{ETNC}\left(\mathbb{Q}(0)_{K}, \mathbb{Z}[G]\right)$ is also valid, then in $K_{0}(\mathbb{Z}[G])$ one has

$$
\left(\mathcal{O}_{K, S}^{\times}\right)-\left|S_{\infty}\right| \cdot(\mathbb{Z}[G])=\left(\operatorname{Pic}\left(\mathcal{O}_{K, S}\right)\right) .
$$

In particular, if $\Lambda$ is any finitely generated subring of $\mathbb{Q}$ for which both $\Lambda \otimes\left(\mathcal{O}_{K, S}^{\times}\right)_{\text {tor }}$ and $\Lambda \otimes \operatorname{Pic}\left(\mathcal{O}_{K, S}\right)$ vanish, then $\Lambda \otimes \mathcal{O}_{K, S}^{\times}$is a free $\Lambda[G]$ module of rank $\left|S_{\infty}\right|$.

Proof. Let $\tau$ denote the a.t.e. $\tau_{0}$ defined in Proposition 4.2.2.

We first assume the hypotheses of claim (i). Then, since $G$ is a $p$-group and the order of $k_{\text {tor }}^{\times}$is prime to $p$, the order of $K_{\text {tor }}^{\times}=H_{\tau \text {,tor }}^{0}$ is also prime to $p$. In addition, Lemma 7.2.2(i) implies that the torsion subgroup of $\left(H_{\tau}^{1}\right)_{J}$ is isomorphic to $\operatorname{Pic}\left(\mathcal{O}_{K^{J}, S}\right)$ for each subgroup $J$ of $G$ and so the stated hypotheses imply that $\tau$ satisfies the hypotheses of Theorem 5.2.1. From Theorem 5.2.1(iii) it therefore follows that $\left(\mathcal{O}_{K, S}^{\times}\right)_{\mathrm{tf}}=H_{\tau, \mathrm{tf}}^{0}$ is a locally-free $\mathbb{Z}[G]$-module if and only if $\operatorname{dim}_{\mathbb{C}}\left(e_{\chi}\left(\mathbb{C} \otimes H_{\tau}^{1}\right)\right)=\operatorname{dim}_{\mathbb{C}}\left(e_{\chi}\left(\mathbb{C} \otimes X_{K, S}\right)\right)$ is independent of $\chi$. But every archimedean place splits completely in $K / k$ (since the condition $p \nmid\left|k_{\text {tor }}^{\times}\right|$implies that $|G|$ is odd) and so the explicit structure of $X_{K, S}$ makes it clear that $\operatorname{dim}_{\mathbb{C}}\left(e_{\chi}\left(\mathbb{C} \otimes X_{K, S}\right)\right)$ is independent of $\chi$ precisely when there exists a unique place of $k$ which ramifies in $K / k$ and the decomposition subgroup of this place is equal to $G$. This proves claim (i). 
Next we assume the hypotheses stated in the first two sentences of claim (ii). Then, since $p$ is odd, all archimedean places of $k$ split completely in $K / k$ and so $H_{\tau, \mathrm{tf}}^{1}=X_{K, S}$ is a free $\mathbb{Z}[G]$-module of rank $\left|S_{\infty}\right|$. This fact combines with Lemma 7.2.2(i) to imply that $\left(\mathbb{Z}_{p} \otimes \operatorname{Pic}\left(\mathcal{O}_{K, S}\right)\right)_{G}=\mathbb{Z}_{p} \otimes\left(H_{\tau, \text { tor }}^{1}\right)_{G}$ is isomorphic to $\mathbb{Z}_{p} \otimes\left(H_{\tau}^{1}\right)_{G \text {,tor }}=\mathbb{Z}_{p} \otimes \operatorname{Pic}\left(\mathcal{O}_{k, S}\right)=0$ and so Nakayama's Lemma implies that $\mathbb{Z}_{p} \otimes \operatorname{Pic}\left(\mathcal{O}_{K, S}\right)=0$. It is thus clear that the $G$-module $H_{\tau, \text { tor }}^{1}=\operatorname{Pic}\left(\mathcal{O}_{K, S}\right)$ is c-t. Since $H_{\tau, \mathrm{tf}}^{1}$ is a free $G$-module it follows that $H_{\tau}^{1}$ is also c-t. By considering Tate cohomology of the sequence (18) this then implies that $H_{\tau}^{0}=\mathcal{O}_{K, S}^{\times}$is c-t.

We now assume in addition that $\operatorname{ETNC}\left(\mathbb{Q}(0)_{K}, \mathbb{Z}[G]\right)$ is valid. Then Proposition 4.2.2 shows that $\chi(\tau)=0$ and so, by the same argument as used to prove Theorem 5.2.1(iv)(a), we deduce that $0=\left(H_{\tau}^{0}\right)-\left(H_{\tau}^{1}\right)=\left(H_{\tau}^{0}\right)-\left(H_{\tau, \text { tor }}^{1}\right)-\left(H_{\tau, \text { tf }}^{1}\right)=$ $\left(\mathcal{O}_{K, S}^{\times}\right)-\left(\operatorname{Pic}\left(\mathcal{O}_{K, S}\right)\right)-\left|S_{\infty}\right|(\mathbb{Z}[G])$ in $K_{0}(\mathbb{Z}[G])$. This is the equality in claim (ii).

Lastly, we fix a ring $\Lambda$ as in the statement of claim (ii). Then $\Lambda \otimes \mathcal{O}_{K, S}^{\times}$is a torsion-free c-t $\Lambda[G]$-module and $\mathbb{Q} \otimes \mathcal{O}_{K, S}^{\times}$is isomorphic to the free $\mathbb{Q}[G]$-module $\mathbb{Q} \otimes X_{K, S}$. Taken together, these facts imply that $\Lambda \otimes \mathcal{O}_{K, S}^{\times}$is a locally-free $\Lambda[G]$-module (cf. the proof of Lemma 3.2.1). But the displayed equality in claim (ii) induces an equality $\left(\Lambda \otimes \mathcal{O}_{K, S}^{\times}\right)=\left|S_{\infty}\right|(\Lambda[G])$ in $K_{0}(\Lambda[G])$ and so locally-free cancellation implies that $\Lambda \otimes \mathcal{O}_{K, S}^{\times}$is indeed a free $\Lambda[G]$-module of rank $\left|S_{\infty}\right|$.

Remark 7.2.4. Explicit unit structures

Let $p$ be an odd prime and $K$ a $p$-power degree abelian extension of $k=\mathbb{Q}$ in which precisely one (non-archimedean) prime $\ell$ ramifies. Then $G:=\operatorname{Gal}(K / \mathbb{Q})$ is necessarily cyclic, the inertial subgroup of $\ell$ is equal to $G$ and for any subgroup $J$ of $G$ the unique place of $K^{J}$ above $\ell$ is principal. Further, it is known that the cardinality of $\operatorname{Pic}\left(\mathcal{O}_{K^{J}, S}\right)=\operatorname{Pic}\left(\mathcal{O}_{K^{J}}\right)$ is coprime to $p$ (cf. [48]) and that $\operatorname{ETNC}\left(\mathbb{Q}(0)_{K}, \mathbb{Z}[G]\right)$ is valid (see Remark 4.2.1). Proposition 7.2.3(ii) therefore specialises to imply that $\left(\mathcal{O}_{K, S}^{\times}\right)-(\mathbb{Z}[G])=\left(\operatorname{Pic}\left(\mathcal{O}_{K}\right)\right)$ in $K_{0}(\mathbb{Z}[G])$. This equality recovers the result of Fröhlich in [50, Th. 5] and the same approach also gives analogous results in the case that $k$ is an imaginary quadratic field of class number one.

7.2.4. $\mathbb{Q}(r)_{K}$ with $r<0$.

Proposition 7.2.5. Let $p$ be an odd prime and $G$ a group of p-power order. Let $S$ denote the (finite) set of places of $k$ comprising $S_{\infty}$ and those places which ramify in $K / k$. 
Fix a strictly negative integer $r$ and assume that the Chern class maps in (10) are bijective for all odd primes $\ell$. Let $s_{1}$ and $s_{2}$ denote the number of real and complex places of $k$ respectively and set $d_{k, r}:=s_{2}+\frac{1}{2}\left(1+(-1)^{r}\right) s_{1}$.

If $p$ does not divide $\left|K_{-2 r}\left(\mathcal{O}_{k, S}\right)\right|$, then the $G$-modules $K_{1-2 r}\left(\mathcal{O}_{K}\right)$ and $K_{-2 r}\left(\mathcal{O}_{K, S}\right)$ are both $c$-t. Further, if $\operatorname{ETNC}\left(\mathbb{Q}(r)_{K}, \mathbb{Z}[G]\right)$ is valid, and we set $\mathbb{Z}^{\prime}:=\mathbb{Z}\left[\frac{1}{2}\right]$, then in $K_{0}\left(\mathbb{Z}^{\prime}[G]\right)$ one has

$$
\left(\mathbb{Z}^{\prime} \otimes K_{1-2 r}\left(\mathcal{O}_{K}\right)\right)-d_{k, r} \cdot\left(\mathbb{Z}^{\prime}[G]\right)=\left(\mathbb{Z}^{\prime} \otimes K_{-2 r}\left(\mathcal{O}_{K, S}\right)\right) .
$$

In particular, if $\Lambda$ is any finitely generated subring of $\mathbb{Q}$ which contains $\mathbb{Z}^{\prime}$ and is such that both $\Lambda \otimes K_{1-2 r}\left(\mathcal{O}_{K}\right)_{\text {tor }}$ and $\Lambda \otimes K_{-2 r}\left(\mathcal{O}_{K, S}\right)$ vanish, then $\Lambda \otimes K_{1-2 r}\left(\mathcal{O}_{K}\right)$ is a free $\Lambda[G]$-module of rank $d_{k, r}$.

Proof. Let $\tau$ denote the a.t.e. $\tau_{r}$ defined in Proposition 4.2.6.

We note first that no archimedean place ramifies in $K / k$ (since $|G|$ is odd) and hence that $\mathbb{Z}^{\prime} \otimes H_{\tau, \mathrm{tf}}^{1}=\left(\bigoplus_{\mathrm{Hom}(K, \mathbb{C})}(2 \pi i)^{-r} \mathbb{Z}^{\prime}\right)^{\mathrm{Gal}(\mathbb{C} / \mathbb{R})}$ is a free $\mathbb{Z}^{\prime}[G]$-module of rank $d_{k, r}$. This fact combines with Lemma 7.2.2(ii) to imply that $\left(\mathbb{Z}_{p} \otimes\right.$ $\left.K_{-2 r}\left(\mathcal{O}_{K, S}\right)\right)_{G}=\mathbb{Z}_{p} \otimes\left(H_{\tau, \text { tor }}^{1}\right)_{G}$ is isomorphic to $\mathbb{Z}_{p} \otimes\left(H_{\tau}^{1}\right)_{G, \text { tor }}=\mathbb{Z}_{p} \otimes K_{-2 r}\left(\mathcal{O}_{k, S}\right)$ $=0$ and so Nakayama's Lemma implies $\mathbb{Z}_{p} \otimes\left(H_{\tau, \text { tor }}^{1}\right) \cong \mathbb{Z}_{p} \otimes K_{-2 r}\left(\mathcal{O}_{K, S}\right)$ vanishes. It is thus clear that the $G$-module $H_{\tau \text {,tor }}^{1}$, and hence also $K_{-2 r}\left(\mathcal{O}_{K, S}\right)$, is c-t. Since $H_{\tau, \mathrm{tf}}^{1}$ is also c-t we deduce that $H_{\tau}^{1}$ is itself c-t. By considering Tate cohomology of the sequence (18) this then implies that $K_{1-2 r}\left(\mathcal{O}_{K}\right)$ is also c-t.

We now assume that $\operatorname{ETNC}\left(\mathbb{Q}(r)_{K}, \mathbb{Z}[G]\right)$ is valid. Then Proposition 4.2.6 implies that $\chi(\tau)$ vanishes and so, by the same argument as used to prove Theorem 5.2.1(iv)(a), one has equalities $0=\left(\mathbb{Z}^{\prime} \otimes H_{\tau}^{0}\right)-\left(\mathbb{Z}^{\prime} \otimes H_{\tau}^{1}\right)=\left(\mathbb{Z}^{\prime} \otimes H_{\tau}^{0}\right)-\left(\mathbb{Z}^{\prime} \otimes\right.$ $\left.H_{\tau, \text { tor }}^{1}\right)-\left(\mathbb{Z}^{\prime} \otimes H_{\tau, \text { tf }}^{1}\right)=\left(\mathbb{Z}^{\prime} \otimes K_{1-2 r}\left(\mathcal{O}_{K}\right)\right)-\left(\mathbb{Z}^{\prime} \otimes K_{-2 r}\left(\mathcal{O}_{K, S}\right)\right)-d_{k, r} \cdot\left(\mathbb{Z}^{\prime}[G]\right)$ in $K_{0}\left(\mathbb{Z}^{\prime}[G]\right)$. This is the displayed equality in the Proposition. Also, the final assertion of the Proposition can be deduced from this equality in just the same way that the final assertion of Proposition 7.2.3(ii) was deduced from the displayed equality in that result.

7.2.5. Elliptic curves. In this section we assume the hypotheses and notation of Proposition 4.3.1. In particular, we assume that condition (B) of Proposition 4.3.1 is satisfied and take (as we may in this case) $\Lambda=\mathbb{Z}^{\prime}:=\mathbb{Z}\left[\frac{1}{2}\right]$. 
Proposition 7.2.6. Under the above conditions we further assume that $G$ is a cyclic group of p-power order and that $p$ does not divide the order of $\amalg\left(E_{/ K^{J}}\right)$ for any non-trivial subgroup $J$ of $G$.

(i) Then the $\mathbb{Z}_{p}[G]$-module $\mathbb{Z}_{p} \otimes E(K)$ is a permutation lattice.

(ii) If $[23$, Conj. $4(\mathrm{ii})]$ is valid for the pair $\left(h^{1}\left(E_{/ K}\right)(1), \mathbb{Q}[G]\right)$, then as $\chi$ varies over $\operatorname{Hom}\left(G, \mathbb{C}^{\times}\right)$, the order of vanishing of $L(E, \chi, s)$ at $s=1$ is a decreasing function of the order of $\chi$. Further, the order of vanishing of $L(E, \chi, s)$ at $s=1$ is independent of $\chi$ if and only if $E(K)_{\mathrm{tf}}$ is a locallyfree $\mathbb{Z}[G]$-module and if this is the case, then $p$ does not divide the order of $\amalg\left(E_{/ K}\right)$.

(iii) Assume that $\left[23\right.$, Conj. 4(ii)] is valid for the pair $\left(h^{1}\left(E_{/ K}\right)(1), \mathbb{Q}[G]\right)$ and that the order of vanishing of $L(E, \chi, s)$ at $s=1$ is independent of $\chi$. Then each of the $\mathbb{Z}^{\prime}[G]$-modules $\mathbb{Z}^{\prime} \otimes E(K), \mathbb{Z}^{\prime} \otimes \amalg\left(E_{/ K}\right), c_{E, \mathbb{Z}^{\prime}}^{0}(K)$ and $c_{E, \mathbb{Z}^{\prime}}^{1}(K)$ is both finitely generated and $c$ - $t$. In addition, if the statement of $\operatorname{ETNC}\left(h^{1}\left(E_{/ K}\right)(1), \mathbb{Z}^{\prime}[G]\right)$ is valid, then in $K_{0}\left(\mathbb{Z}^{\prime}[G]\right)$ one has

$$
\left(1-\psi^{*}\right)\left(\mathbb{Z}^{\prime} \otimes E(K)\right)=\left(\mathbb{Z}^{\prime} \otimes \amalg\left(E_{/ K}\right)\right)-\left(c_{E, \mathbb{Z}^{\prime}}^{0}(K)\right)+\left(c_{E, \mathbb{Z}^{\prime}}^{1}(K)\right)
$$

where $\psi^{*}$ is the involution of $K_{0}\left(\mathbb{Z}^{\prime}[G]\right)$ defined in $\S 2.5$. In particular, if $\Lambda$ is any finitely generated subring of $\mathbb{Q}$ in which 2 is invertible and the modules $\Lambda \otimes E(K)_{\text {tor }}, \Lambda \otimes \amalg\left(E_{/ K}\right), \Lambda \otimes_{\mathbb{Z}^{\prime}} c_{E, \mathbb{Z}^{\prime}}^{0}(K)$ and $\Lambda \otimes_{\mathbb{Z}^{\prime}} c_{E, \mathbb{Z}^{\prime}}^{1}(K)$ all vanish, then the $\Lambda[G]$-modules $\Lambda \otimes E(K)$ and $\Lambda \otimes E(K)^{*}$ are isomorphic.

Proof. We let $\tau$ be the a.t.e. $\tau_{E, \mathbb{Z}^{\prime}}$ defined in Proposition 4.3 .1 and for each $G$ module $M$ set $M^{\prime}:=\mathbb{Z}^{\prime} \otimes M$. Then Lemma 7.2.2(ii) implies that the torsion subgroup of $\left(H_{\tau}^{1}\right)_{J}$ is isomorphic to $\amalg\left(E_{/ K^{J}}\right)^{\vee^{\prime}} \oplus c_{E, \mathbb{Z}^{\prime}}^{1}\left(K^{J}\right)$ for each subgroup $J$ of $G$. Further, the module $H_{\tau, \text { tor }}^{0}=E(K)_{\text {tor }}^{\prime} \oplus\left(E(K)_{\text {tor }}\right)^{\vee \prime} \oplus c_{E, \mathbb{Z}^{\prime}}^{0}(K)$ is of order prime to $p$ and hence is c-t. The stated hypotheses therefore imply that $\tau$ satisfies the hypotheses of Theorem 5.2.1 and so Theorem 5.2.1(i) implies that $\mathbb{Z}_{p} \otimes_{\mathbb{Z}^{\prime}} H_{\tau}^{0}=\mathbb{Z}_{p} \otimes E(K)$ is a permutation lattice of the form described in (23). This proves claim (i).

Now if $\left[23\right.$, Conj. 4(ii)] is valid for $\left(h^{1}\left(E_{/ K}\right)(1), \mathbb{Q}[G]\right)$, then for each character $\chi$ in $\operatorname{Hom}\left(G, \mathbb{C}^{\times}\right)$the order of vanishing $\operatorname{ord}_{s=1} L(E, \chi, s)$ of $L(E, \chi, s)$ at $s=1$ is equal to $\operatorname{dim}_{\mathbb{C}}\left(e_{\bar{\chi}}(\mathbb{C} \otimes E(K))\right)$. But the isomorphism (23) implies that the $\mathbb{C}[G]$-module $\mathbb{C} \otimes E(K)$ is isomorphic to $\bigoplus_{J \leq G} \mathbb{C}[G / J]^{\left\langle n_{J}\right\rangle}$ and so it is clear that $\operatorname{ord}_{s=1} L(E, \chi, s)$ is a decreasing function of the order of $\chi$. For 
the same reason, Theorem 5.2.1(iii) implies that $\operatorname{ord}_{s=1} L(E, \chi, s)$ is independent of $\chi$ precisely when $E(K)_{\mathrm{tf}}^{\prime}$ is a locally-free $\mathbb{Z}^{\prime}[G]$-module. Further, if $E(K)_{\mathrm{tf}}^{\prime}$ is a locally-free $\mathbb{Z}^{\prime}[G]$-module, then it, and hence also its linear dual $E(K)^{* \prime}$, is a projective $\mathbb{Z}^{\prime}[G]$-module and so the image under $\mathbb{Z}^{\prime} \otimes$ - of the exact sequence (11) splits to give an isomorphism $\operatorname{Sel}\left(E_{/ K}\right)^{\prime} \cong \amalg\left(E_{/ K}\right)^{\vee^{\prime}} \oplus E(K)^{* \prime}$. The module of $G$-coinvariants $\left(\amalg\left(E_{/ K}\right)^{\vee^{\prime}}\right)_{G}$ is therefore isomorphic to a subgroup of $\operatorname{Sel}\left(E_{/ K}\right)_{G, \text { tor }}^{\prime} \subseteq\left(H_{\tau}^{1}\right)_{G \text {,tor }} \cong \amalg\left(E_{/ \mathbb{Q}}\right)^{\vee^{\prime}} \oplus c_{E, \mathbb{Z}^{\prime}}^{1}(\mathbb{Q})$. Since, by assumption, $\left|\amalg\left(E_{/ \mathbb{Q}}\right)\right|$ is prime to $p$ and, by Proposition $4.3 .1,\left|c_{E, \mathbb{Z}^{\prime}}^{1}(\mathbb{Q})\right|$ is prime to $p$, it follows that $\left.\mid \amalg\left(E_{/ K}\right)^{\vee \prime}\right)_{G} \mid$ is also prime to $p$ and hence (by Nakayama's Lemma and the fact that $p$ is odd) that $\left|\amalg\left(E_{/ K}\right)\right|$ is prime to $p$. This proves claim (ii).

We now assume the hypotheses of claim (iii). Then claim (ii) implies that the $\mathbb{Z}^{\prime}[G]$-module $E(K)_{\mathrm{tf}}^{\prime}$, and hence also $H_{\tau, \mathrm{tf}}^{1} \cong \operatorname{Hom}_{\mathbb{Z}}\left(E(K)_{\mathrm{tf}}, \mathbb{Z}\right)^{\prime}=E(K)^{* \prime}$, is locally-free and therefore c-t. Since $E(K)_{\text {tor }}^{\prime}$ is also c-t (by Proposition 4.3.1 and the fact that $G$ is a $p$-group) we deduce that the module $E(K)^{\prime}$ is itself c-t. From claim (ii) we also know that the $\mathbb{Z}^{\prime}[G]$-module $\amalg\left(E_{/ K}\right)^{\vee^{\prime}}$ has order prime to $p$ and so is c-t and Proposition 4.3.1 implies that the same is true for the modules $c_{E, \mathbb{Z}^{\prime}}^{0}(K)$ and $c_{E, \mathbb{Z}^{\prime}}^{1}(K)$. Further, if $\operatorname{ETNC}\left(h^{1}\left(E_{/ K}\right)(1), \mathbb{Z}^{\prime}[G]\right)$ is valid, then Proposition 4.3.1 implies that $\chi(\tau)$ vanishes and so Theorem 5.2.1(iv)(a) implies that in $K_{0}\left(\mathbb{Z}^{\prime}[G]\right)$ there are equalities

$$
\begin{aligned}
& 0=\left(H_{\tau}^{0}\right)-\left(H_{\tau}^{1}\right)=\left(H_{\tau}^{0}\right)-\left(H_{\tau, \text { tor }}^{1}\right)-\left(H_{\tau, \mathrm{tf}}^{1}\right)= \\
&\left(E(K)^{\prime}\right)+\left(\left(E(K)_{\mathrm{tor}}\right)^{\vee \prime}\right)+\left(c_{E, \mathbb{Z}^{\prime}}^{0}(K)\right) \\
& \quad-\left(\amalg\left(E_{/ K}\right)^{\vee \prime}\right)-\left(c_{E, \mathbb{Z}^{\prime}}^{1}(K)\right)-\left(E(K)^{* \prime}\right) .
\end{aligned}
$$

To deduce the displayed equality of claim (iii) we thus need only note that the Cassels-Tate pairing induces an isomorphism of $G$-modules $\amalg\left(E_{/ K}\right)^{\vee} \cong \amalg\left(E_{/ K}\right)$ and that if $N$ is any finitely generated $\mathbb{Z}^{\prime}[G]$-module for which both $N_{\text {tor }}$ and $N_{\text {tf }}$ are c-t, then $N^{*}$ and $\left(N_{\text {tor }}\right)^{\vee}$ are also both c-t and in $K_{0}\left(\mathbb{Z}^{\prime}[G]\right)$ one has $\psi^{*}(N)=\left(N^{*}\right)-\left(\left(N_{\text {tor }}\right)^{\vee}\right)$.

Finally we fix $\Lambda$ as in the statement of claim (iii). Then the above displayed equality implies that there is an equality $(\Lambda \otimes E(K))=\left(\Lambda \otimes E(K)^{*}\right)$ in $K_{0}(\Lambda[G])$. But $\Lambda \otimes E(K)$, and hence also $\Lambda \otimes E(K)^{*}=\operatorname{Hom}_{\Lambda}(\Lambda \otimes E(K), \Lambda)$, is a locallyfree $\Lambda[G]$-module (by claim (ii)) and so the equality $(\Lambda \otimes E(K))=\left(\Lambda \otimes E(K)^{*}\right)$ combines with locally-free cancellation to imply that the $\Lambda[G]$-modules $\Lambda \otimes E(K)$ and $\Lambda \otimes E(K)^{*}$ are isomorphic. 


\section{Strong main conjectures}

In this section we prove Theorem 5.1.1(iv) and Theorem 5.2.1(iv)(c) and also discuss some explicit arithmetic applications of these results. We assume throughout that $G$ is abelian.

8.1. Preliminaries. We first quickly recall some details concerning the determinant functor used by Knudsen and Mumford in [67]. For any commutative associative unital ring $R$ we write $\mathcal{P}(R)$ for the category of graded invertible $R$ modules and isomorphisms of such and $\operatorname{Det}_{R}$ for the determinant functor of loc. cit.

We let $\Lambda$ be a finitely generated subring of $\mathbb{Q}$. Then $[23, \S 2.5]$ implies that $K_{0}(\Lambda[G], \mathbb{R}[G])$ can be identified with the multiplicative group of invertible $\Lambda[G]$ lattices in $\mathbb{R}[G]$. In addition, the reduced norm map $\operatorname{Nrd}_{\mathbb{R}[G]}$ is bijective (since $G$ is abelian) and, with respect to the above identification, the equality (3) implies that

$$
\delta_{\Lambda}(x)=\partial_{\Lambda[G], \mathbb{R}}^{1}\left(\operatorname{Nrd}_{\mathbb{R}[G]}^{-1}(x)\right)=\Lambda[G] \cdot x \subset \mathbb{R}[G]
$$

for every $x$ in $\zeta(\mathbb{R}[G])^{\times}$. Further, the element $[\epsilon, \psi]$ defined in (6) (with $\mathfrak{A}=\Lambda[G]$ and $E=\mathbb{R}$ ) can be computed in the following manner. The isomorphism $\psi$ induces an isomorphism in $\mathcal{P}(\mathbb{R}[G])$

$$
\vartheta_{\psi}: \mathbb{R} \otimes_{\Lambda}\left(\operatorname{Det}_{\Lambda[G]} C^{\bullet}\right) \stackrel{\sim}{\longrightarrow}(\mathbb{R}[G], 0)
$$

by means of the composite

$$
\begin{aligned}
& \mathbb{R} \otimes_{\Lambda}\left(\operatorname{Det}_{\Lambda[G]} C^{\bullet}\right) \\
\stackrel{\sim}{\longrightarrow} & \operatorname{Det}_{\mathbb{R}[G]}\left(\mathbb{R} \otimes_{\Lambda} H^{0}\left(C^{\bullet}\right)\right) \otimes_{\mathcal{P}(\mathbb{R}[G])} \operatorname{Det}_{\mathbb{R}[G]}\left(\mathbb{R} \otimes_{\Lambda} H^{1}\left(C^{\bullet}\right)\right)^{-1} \\
\stackrel{\sim}{\longrightarrow} & \operatorname{Det}_{\mathbb{R}[G]}\left(\mathbb{R} \otimes_{\Lambda} H^{1}\left(C^{\bullet}\right)\right) \otimes_{\mathcal{P}(\mathbb{R}[G])} \operatorname{Det}_{\mathbb{R}[G]}\left(\mathbb{R} \otimes_{\Lambda} H^{1}\left(C^{\bullet}\right)\right)^{-1} \\
\stackrel{\sim}{\longrightarrow} & \mathbb{R}[G], 0) .
\end{aligned}
$$

Here the first, resp. second, resp. third, isomorphism is induced by [67, Rem. b) following Th. 2] and the fact that the algebra $\mathbb{R}[G]$ is semisimple, resp. the isomorphism $\operatorname{Det}_{\mathbb{R}[G]}(\psi) \otimes \mathrm{id}$, resp. the evaluation pairing on the module $\operatorname{Det}_{\mathbb{R}[G]}\left(\mathbb{R} \otimes_{\Lambda} H^{1}\left(C^{\bullet}\right)\right)$. The element $[\epsilon, \psi]$ is then equal to the invertible $\Lambda[G]$ submodule of $\mathbb{R}[G]$ which underlies $\vartheta_{\psi}\left(\operatorname{Det}_{\Lambda[G]} C^{\bullet}\right)$. 


\section{Remark 8.1.1. Explicit computations}

Assume the conditions and notation of Lemma 3.2.1. If $G$ is abelian, then the above observations regarding the $\Lambda[G]$-lattice $[\epsilon, \psi]$ lead to the following method of explicit computation. We set $P_{1}^{*}:=\operatorname{Hom}_{\Lambda[G]}\left(P^{1}, \Lambda[G]\right), P_{\mathbb{R}}^{0}:=\mathbb{R} \otimes_{\Lambda} P^{0}$, $P_{\mathbb{R}}^{1}:=\mathbb{R} \otimes_{\Lambda} P^{1}$ and $P_{1, \mathbb{R}}^{*}:=\mathbb{R} \otimes_{\Lambda} P_{1}^{*}$. It is enough to compute $\mathbb{Z}_{(p)} \otimes_{\Lambda}[\epsilon, \psi]$ at each prime $p \in \operatorname{Spec}(\Lambda)$. But, for each such $p$, the $\mathbb{Z}_{(p)}[G]$-modules $\mathbb{Z}_{(p)} \otimes_{\Lambda} P^{0}$ and $\mathbb{Z}_{(p)} \otimes_{\Lambda} P_{1}^{*}$ are both free of the same rank, $n$ say. After choosing ordered bases $\left\{a_{i}\right\}_{1 \leq i \leq n}$ and $\left\{b_{j}^{*}\right\}_{1 \leq j \leq n}$ for each of them, and identifying $\wedge_{\mathbb{R}[G]}^{n} P_{1, \mathbb{R}}^{*}$ with $\operatorname{Hom}_{\mathbb{R}[G]}\left(\wedge_{\mathbb{R}[G]}^{n} P_{\mathbb{R}}^{1}, \mathbb{R}[G]\right)$ in the natural way, $\mathbb{Z}_{(p)} \otimes[\epsilon, \psi]$ is the $\mathbb{Z}_{(p)}[G]$-sublattice of $\mathbb{R}[G]$ generated by the image of the element $\wedge_{i=1}^{n} \iota\left(a_{i}\right) \otimes_{\mathbb{R}[G]} \wedge_{j=1}^{n} b_{j}^{*}$ under the evaluation pairing

$$
\wedge_{\mathbb{R}[G]}^{n} P_{\mathbb{R}}^{1} \otimes_{\mathbb{R}[G]} \operatorname{Hom}_{\mathbb{R}[G]}\left(\wedge_{\mathbb{R}[G]}^{n} P_{\mathbb{R}}^{1}, \mathbb{R}[G]\right) \rightarrow \mathbb{R}[G]
$$

(where $\iota$ is the isomorphism $P_{\mathbb{R}}^{0} \rightarrow P_{\mathbb{R}}^{1}$ defined in Lemma 3.2.1(i)).

8.2. The proof of Theorem 5.1.1(iv). For each $\chi \in \operatorname{Irr}(G)$ we set $e_{\chi}:=$ $|G|^{-1} \sum_{g \in G} \chi(g) g^{-1} \in \mathbb{C}[G]$. We then obtain an idempotent of $\mathbb{Q}[G]$ by setting

$$
e_{\tau}:=\sum_{\chi \in \Upsilon_{\tau}} e_{\chi}
$$

where $\Upsilon_{\tau}$ is the subset of $\operatorname{Irr}(G)$ comprising characters $\chi$ with $e_{\chi}\left(\mathbb{C} \otimes_{\Lambda} H_{\tau}^{0}\right)=0$ (and $e_{\tau}$ belongs to $\mathbb{Q}[G]$ since $\Upsilon_{\tau}$ is a union of orbits under the natural action of $\operatorname{Aut}_{\mathbb{Q}}(\mathbb{C})$ on $\left.\operatorname{Irr}(G)\right)$. We also define

$$
\mathcal{L}_{\tau}:=\mathcal{L}_{\tau}^{*} e_{\tau} \in \mathbb{R}[G]^{\times} e_{\tau}=\left(\mathbb{R}[G] e_{\tau}\right)^{\times}
$$

and to study this element we set $\mathfrak{A}:=\Lambda[G] e_{\tau}$ and $A:=\mathbb{Q}[G] e_{\tau}$. For any $\mathfrak{A}$ module $W$ we set $W^{*}:=\operatorname{Hom}_{\Lambda}(W, \Lambda)$, endowed with its natural structure as $\mathfrak{A}$-module (that is, $a(\theta)(w)=\theta(a w)$ ). For brevity we shall also often identify graded invertible $\mathfrak{A}$-modules of the form $(I, 0)$ with the underlying invertible module $I$.

For any commutative ring $R$ and finitely generated $R$-module $N$ we write $\operatorname{Fit}_{R}(N)$ for the (initial) Fitting ideal of $N$.

Theorem 8.2.1. Assume that $G$ is abelian and that $\chi(\tau)$ vanishes.

(i) If $\Psi^{\bullet}$ is any complex as in (18), then in $(\mathbb{R}[G], 0)$ one has

$$
\left(\Lambda[G] \cdot \mathcal{L}_{\tau}^{*}, 0\right)=\vartheta_{\lambda_{\tau}}\left(\operatorname{Det}_{\Lambda[G]} \Psi^{\bullet}\right)
$$


(ii) $\mathcal{L}_{\tau}$ belongs to $\mathbb{Q}[G]$.

(iii) If $\left(H_{\tau}^{0}\right)_{\text {tor }}$ is $\Lambda[G]$-perfect, then $\operatorname{Fit}_{\Lambda[G]}\left(\left(H_{\tau}^{0}\right)_{\text {tor }}\right) \cdot \mathcal{L}_{\tau}=\operatorname{Fit}_{\Lambda[G]}\left(H_{\tau}^{1}\right)$.

Proof. Claim (i) is an easy consequence of the explicit descriptions of $\left[\epsilon_{\tau}, \lambda_{\tau}\right]$ and $\delta_{\Lambda}\left(\mathcal{L}_{\tau}^{*}\right)$ given by the discussion in $\S 8.1$. Regarding claim (ii) we first observe that, since $\mathbb{C} \otimes_{\Lambda} H_{\tau}^{1}$ is isomorphic to $\mathbb{C} \otimes_{\Lambda} H_{\tau}^{0}$ as a $\mathbb{C}[G]$-module, for each $\psi \in \Upsilon_{\tau}$ the space $\operatorname{Hom}_{\mathbb{C}[G]}\left(\mathbb{C}[G] e_{\psi}, \mathbb{C} \otimes_{\Lambda} H_{\tau}^{1}\right)$ vanishes and hence, in the notation of Theorem 5.1.1(i), one has $A_{\varphi}^{\tau}(\psi)=\left(\mathcal{L}_{\tau, \psi}^{*}\right)^{-1}$ for each such $\psi$. Claim (ii) thus follows directly from Lemma 6.1.1 and our assumption that the Euler characteristic $\chi(\tau)$ vanishes.

We now turn to claim (iii). If $W$ is any $\Lambda[G]$-module, then we endow $W \otimes_{\Lambda} \mathfrak{A}$ with its natural structure as $(\Lambda[G], \mathfrak{A})$-bimodule (so, in particular, each element $g$ of $G$ acts on the tensor via $\left.g \otimes_{\Lambda} g^{-1}\right)$. We also let $W^{\mathfrak{A}}$ and $W_{\mathfrak{A}}$ denote the associated $\mathfrak{A}$-modules $\left(W \otimes_{\Lambda} \mathfrak{A}\right)^{G}$ and $\left(W \otimes_{\Lambda} \mathfrak{A}\right)_{G} \cong W \otimes_{\Lambda[G]} \mathfrak{A}$. We recall that if $N$ is any finite $\mathfrak{A}$-module of projective dimension at most one, then $\operatorname{Fit}_{\mathfrak{A}}(N)$ is an invertible ideal of $\mathfrak{A}$ and in $\mathcal{P}(\mathfrak{A})$ one has

$$
\operatorname{Det}_{\mathfrak{A}}(N)=\left(\operatorname{Fit}_{\mathfrak{A}}(N)^{-1}, 0\right) .
$$

We fix a concrete complex $\Psi^{\bullet}$ as in claim (i) and set $\Psi_{\mathfrak{A}}^{\bullet}:=\Psi^{\bullet} \otimes_{\Lambda[G]} \mathfrak{A}$. Then, since each term of $\Psi^{\bullet}$ is a perfect $\Lambda[G]$-module, there is a natural isomorphism in $D^{\text {perf }}(\mathfrak{A})$ between $\Psi_{\mathfrak{A}}^{\bullet}$ and $\Psi^{\bullet} \otimes_{\Lambda[G]}^{\mathbb{L}} \mathfrak{A}$ (see the proof of Lemma 8.2.2 below). Claim (i) therefore implies that in $A$ one has

$$
\begin{aligned}
\mathcal{L}_{\tau} \cdot \mathfrak{A} & =\vartheta_{\lambda_{\tau}}\left(\operatorname{Det}_{\Lambda[G]} \Psi^{\bullet}\right) \mathfrak{A} \\
& =\vartheta_{\tau}^{0}\left(\left(\operatorname{Det}_{\Lambda[G]} \Psi^{\bullet}\right) \otimes_{\Lambda[G]} \mathfrak{A}\right) \\
& =\vartheta_{\tau}^{0}\left(\operatorname{Det}_{\mathfrak{A}}\left(\Psi^{\bullet} \otimes_{\Lambda[G]}^{\mathbb{L}} \mathfrak{A}\right)\right) \\
& =\vartheta_{\tau}^{0}\left(\operatorname{Det}_{\mathfrak{A}} \Psi_{\mathfrak{A}}^{\bullet},\right.
\end{aligned}
$$

where $\vartheta_{\tau}^{0}:=\vartheta_{\lambda_{\tau}} \otimes_{\mathbb{R}[G]} \mathbb{R}[G] e_{\tau}$.

To compute this expression we observe that the same kind of argument as used just after (18) gives an exact sequence of $\mathfrak{A}$-modules

$$
0 \rightarrow H^{0, \mathfrak{A}} \rightarrow \Psi_{\mathfrak{A}}^{0} \rightarrow \Psi_{\mathfrak{A}}^{1} \rightarrow H_{\mathfrak{A}}^{1} \rightarrow 0 .
$$

where we set $H^{i}:=H_{\tau}^{i}$ for $i=0,1$. Now our choice of $e:=e_{\tau}$ implies that the modules $H_{\Lambda}^{0, \mathfrak{A}}$ and $H_{\Lambda, \mathfrak{A}}^{1}$ in this sequence are finite. This implies both that $H_{\Lambda}^{0, \mathfrak{A}}=T^{0, \mathfrak{A}}$, where we set $T^{0}:=H_{\text {tor }}^{0}$, and also that $\mathbb{R} \otimes_{\Lambda} \Psi_{\mathfrak{A}}^{\bullet}$ is acyclic so 
that the isomorphism $\vartheta_{\tau}^{0}$ in $(25)$ is the isomorphism $\mathbb{R} \otimes_{\Lambda}\left(\operatorname{Det}_{\mathfrak{A}} \Psi_{\mathfrak{A}}^{\bullet} \cong(\mathbb{R}[G] e, 0)\right.$ that is induced by scalar extension and the obvious equality $\operatorname{Det}_{\mathbb{R}[G]}\left(\mathbb{R} \otimes_{\Lambda} \Psi_{\mathfrak{A}}^{\bullet}\right)=$ $\operatorname{Det}_{\mathbb{R}[G] e}(0)=(\mathbb{R}[G] e, 0)$.

We now assume that the $\Lambda[G]$-module $T^{0}$ is perfect and recall that the $\Lambda[G]$ modules $\Psi^{0}$ and $\Psi^{1}$ are also perfect. Then (since $H^{0, \mathfrak{A}}=T^{0, \mathfrak{A}}$ ) Lemma 8.2.2 below implies that the first three modules in (26) are of projective dimension at most one. The exactness of (26) then implies that the projective dimension of the $\mathfrak{A}$-module $H_{\mathfrak{A}}^{1}$ is finite and hence (by the argument of Lemma 8.2.2) at most one. When taken in conjunction with [67, Rem. b) following Th. 2] and equalities of the form (24), the exact sequence (26) thus implies that

$$
\vartheta_{\tau}^{0}\left(\operatorname{Det}_{\mathfrak{A}} \Psi_{\mathfrak{A}}^{\bullet}\right)=\operatorname{Fit}_{\mathfrak{A}}\left(T^{0, \mathfrak{A}}\right)^{-1} \operatorname{Fit}_{\mathfrak{A}}\left(H_{\mathfrak{A}}^{1}\right) \subset A .
$$

Now Fitting ideals commute with scalar extension and so both

$$
\operatorname{Fit}_{\mathfrak{A}}\left(H_{\mathfrak{A}}^{1}\right)=\operatorname{Fit}_{\Lambda[G]}\left(H^{1}\right) \mathfrak{A}=\operatorname{Fit}_{\Lambda[G]}\left(H^{1}\right) e
$$

and

$$
\operatorname{Fit}_{\mathfrak{A}}\left(T^{0, \mathfrak{A}}\right)=\operatorname{Fit}_{\mathfrak{A}}\left(T_{\mathfrak{A}}^{0}\right)=\operatorname{Fit}_{\Lambda[G]}\left(T^{0}\right) e .
$$

Putting things together, we deduce from (25) that

$$
\operatorname{Fit}_{\Lambda[G]}\left(T^{0}\right) \cdot \mathcal{L}_{\tau}=\operatorname{Fit}_{\Lambda[G]}\left(H^{1}\right) e,
$$

and so it is enough to prove

$$
\operatorname{Fit}_{\Lambda[G]}\left(H^{1}\right) e=\operatorname{Fit}_{\Lambda[G]}\left(H^{1}\right) .
$$

Hence, since $1=e+(1-e)$, it suffices to prove $\operatorname{Fit}_{\Lambda[G]}\left(H^{1}\right)(1-e)=0$, or equivalently that in $\mathbb{C}[G]$ one has $\operatorname{Fit}_{\Lambda[G]}\left(H^{1}\right) e_{\chi}=0$ for every $\chi \in \operatorname{Irr}(G) \backslash \Upsilon_{\tau}$. To do this we fix such a character $\chi$, set $\mathfrak{A}_{\chi}:=\Lambda[\chi][G] e_{\chi}$ (where $\Lambda[\chi]$ denotes the ring generated over $\Lambda$ by the values of $\chi$ ), and choose a resolution of the $\Lambda[G]$-module $H^{1}$ of the form $\Lambda[G]^{m} \stackrel{\theta}{\rightarrow} \Lambda[G]^{n} \rightarrow H^{1} \rightarrow 0$. This sequence induces an exact sequence

$$
\mathfrak{A}_{\chi}^{m} \stackrel{\theta_{\chi}}{\longrightarrow} \mathfrak{A}_{\chi}^{n} \rightarrow H_{\chi}^{1} \rightarrow 0
$$

where $\theta_{\chi}=\theta \otimes_{\Lambda[G]} \mathfrak{A}_{\chi}$ and $H_{\chi}^{1}:=H^{1} \otimes_{\Lambda[G]} \mathfrak{A}_{\chi}$. The latter sequence implies that $\operatorname{Fit}_{\Lambda[G]}\left(H^{1}\right) \cdot \mathfrak{A}_{\chi}=\operatorname{Fit}_{\mathfrak{A}_{\chi}}\left(H_{\chi}^{1}\right)$ is equal to the image $I\left(\theta_{\chi}\right)$ of $\wedge_{\mathfrak{A}_{\chi}}^{n} \theta_{\chi}$ in $\wedge_{\mathfrak{A}_{\chi}}^{n}\left(\mathfrak{A}_{\chi}^{n}\right) \cong$ $\mathfrak{A}_{\chi}$. But, since $\chi \notin \Upsilon_{\tau}$, the $\mathfrak{A}_{\chi}$-rank of $H_{\chi}^{1}$ is at least one and so the exactness of the last displayed sequence also implies that the $\mathfrak{A}_{\chi}$-rank of $\operatorname{im}\left(\theta_{\chi}\right)$ is at most $n-1$. The module $\wedge_{\mathfrak{A}_{\chi}}^{n} \operatorname{im}\left(\theta_{\chi}\right)$ is thus finite and so therefore is its image $I\left(\theta_{\chi}\right)$ in $\mathfrak{A}_{\chi}$. But $\mathfrak{A}_{\chi}$ is torsion-free and so $I\left(\theta_{\chi}\right)=0$, as required. 
Lemma 8.2.2. If $N$ is a perfect $\Lambda[G]$-module, then the $\mathfrak{A}$-modules $N_{\mathfrak{A}}$ and $N^{\mathfrak{A}}$ are isomorphic and have projective dimension at most one.

Proof. If $\mathfrak{B}$ is any $\Lambda$-order, then for each prime $p \in \operatorname{Spec}(\Lambda)$ the ring $\mathbb{Z}_{p} \otimes \mathfrak{B}$ is a product of local rings of Krull dimension one. The result of [4, Th. 1.9] therefore implies that the projective dimension of any finitely generated $\mathfrak{B}$-module is either infinite or at most one.

In particular, our assumption that $N$ is $\Lambda[G]$-perfect implies that there exists an exact sequence of finitely generated $\Lambda[G]$-modules $0 \rightarrow P \rightarrow Q \rightarrow N \rightarrow 0$ in which $P$ and $Q$ are both projective. Now $\operatorname{Tor}_{\Lambda[G]}^{1}(Q, \mathfrak{A})=0$ and so there is an associated exact sequence of $\mathfrak{A}$-modules $0 \rightarrow \operatorname{Tor}_{\Lambda[G]}^{1}(N, \mathfrak{A}) \rightarrow P_{\mathfrak{A}} \rightarrow Q_{\mathfrak{A}} \rightarrow$ $N_{\mathfrak{A}} \rightarrow 0$. But $\operatorname{Tor}_{\Lambda[G]}^{1}(N, \mathfrak{A})$ is finite and $P_{\mathfrak{A}}$ is $\Lambda$-torsion-free and so one must have $\operatorname{Tor}_{\Lambda[G]}^{1}(N, \mathfrak{A})=0$. Since both $Q_{\mathfrak{A}}$ and $P_{\mathfrak{A}}$ are projective $\mathfrak{A}$-modules the latter exact sequence therefore implies that the $\mathfrak{A}$-module $N_{\mathfrak{A}}$ has projective dimension at most one. (This fact lies behind the natural isomorphism in $D^{\text {perf }}(\mathfrak{A})$ between $\Psi_{\mathfrak{A}}^{\bullet}$ and $\Psi^{\bullet} \otimes_{\Lambda[G]}^{\mathbb{L}} \mathfrak{A}$ that is used in (25).)

Finally we note that the $G$-module $N \otimes_{\Lambda} \mathfrak{A}$ is c-t and hence that the tautological exact sequence of $\mathfrak{A}$-modules

$$
0 \rightarrow \hat{H}^{-1}\left(G, N \otimes_{\Lambda} \mathfrak{A}\right) \rightarrow N_{\mathfrak{A}} \rightarrow N^{\mathfrak{A}} \rightarrow \hat{H}^{0}\left(G, N \otimes_{\Lambda} \mathfrak{A}\right) \rightarrow 0
$$

induces an isomorphism $N_{\mathfrak{A}} \cong N^{\mathfrak{A}}$.

\section{Remark 8.2.3. Perfection}

Regarding Theorem 8.2.1(iii), we note that the module $\left(H_{\tau}^{0}\right)_{\text {tor }}$ is $\Lambda[G]$-perfect if the order of $\hat{H}^{i}\left(J,\left(H_{\tau}^{0}\right)_{\text {tor }}\right)$ is invertible in $\Lambda$ for every subgroup $J$ of $G$ and every integer $i$ and hence a fortior $i$ if the highest common factor of $|G|$ and $\left|\left(H_{\tau}^{0}\right)_{\text {tor }}\right|$ is invertible in $\Lambda$.

Remark 8.2.4. Tate motives

We assume that $k$ is totally real and that $K$ is a CM field and let $\kappa$ denote the (unique) non-trivial element of $\operatorname{Gal}\left(K / K^{+}\right)$where $K^{+}$is the maximal real subfield of $K$. For each integer $r$ with $r \leq 0$ and each character $\psi \in \operatorname{Irr}(G)$ the function $L_{S}(\psi, s)$ is holomorphic at $s=r$ and so we may define $L_{K / k, S}(r)$ to be the (unique) element of $\mathbb{R}[G]$ with $L_{K / k, S}(r)_{\psi}=L_{S}(\psi, r)$ for every character $\psi$. We set $\mathbb{Z}^{\prime}:=\mathbb{Z}\left[\frac{1}{2}\right]$ and $N^{\prime}:=\mathbb{Z}^{\prime} \otimes N$ for each $G$-module $N$. 
- Set $\tau:=\tau_{0}$ (as in $\S 4.2 .1$ ). Then [94, Chap. I, Prop. 3.4] implies that $\mathcal{L}_{\tau}=L_{K / k, S}(0)^{\#}$. Also, $H_{\tau, \text { tor }}^{0}$ is the (cyclic) group $\mu_{K}$ of roots of unity in $K$ and so $\operatorname{Fit}_{\mathbb{Z}[G]}\left(H_{\tau, \text { tor }}^{0}\right)=\operatorname{Ann}_{\mathbb{Z}[G]}\left(\mu_{K}\right)$. We assume that $\mu_{K}^{\prime}$ is a perfect $\mathbb{Z}^{\prime}[G]$-module. Then Theorem 8.2.1(iii) implies $\operatorname{Ann}_{\mathbb{Z}^{\prime}[G]}\left(\mu_{K}^{\prime}\right) \cdot L_{K / k, S}(0)^{\#}=\operatorname{Fit}_{\mathbb{Z}^{\prime}[G]}\left(\left(H_{\tau}^{1}\right)^{\prime}\right)$. If now $\kappa$ is contained in the decomposition subgroup of every place $v$ in $S$ (so $K / k$ is 'nice' in the terminology of Greither - see [52] or [53, §3]), then $e_{\tau}=$ $e_{-}:=(1-\kappa) / 2 \in \mathbb{Z}^{\prime}[G]$ and it is easy to check that $e_{-}\left(H_{\tau}^{1}\right)^{\prime}=e_{-} \operatorname{Pic}\left(\mathcal{O}_{K}\right)^{\prime}$. Thus, in this case, Theorem 8.2.1(iii) specializes to show that the validity of $\operatorname{ETNC}\left(\mathbb{Q}(0)_{K}, \mathbb{Z}^{\prime}[G]\right)$ implies the following refinement of Brumer's Conjecture

$$
\operatorname{Ann}_{\mathbb{Z}^{\prime}[G]}\left(\mu_{K}^{\prime}\right) \cdot L_{K / k, S}(0)^{\#}=\operatorname{Fit}_{\mathbb{Z}^{\prime}[G] e_{-}}\left(e_{-} \operatorname{Pic}\left(\mathcal{O}_{K}\right)^{\prime}\right) .
$$

Greither [54] has in fact recently refined this approach to show that for any CM abelian extension $K / k$ the validity of $\operatorname{ETNC}\left(\mathbb{Q}(0)_{K}, \mathbb{Z}^{\prime}[G]\right)$ implies a similarly explicit formula for $\mathrm{Fit}_{\mathbb{Z}^{\prime}[G] e_{-}}\left(e_{-}\left(\operatorname{Pic}\left(\mathcal{O}_{K}\right)^{\vee}\right)^{\prime}\right)$.

- Set $\tau:=\tau_{1}$ (as in $\left.\S 4.2 .2\right)$. Then $e_{\tau_{1}}=0$ unless $k=\mathbb{Q}$ in which case $e_{\tau_{1}}=e_{1_{G}}$ where $1_{G}$ is the trivial character of $G$. In particular, in this case the equality of Theorem 8.2.1(iii) with $\Lambda=\mathbb{Z}$ is easy to verify directly (but see Remark 8.2.6 below).

- Set $\tau:=\tau_{r}$ with $r<0$ (as in $\left.\S 4.2 .3\right)$. Then $e_{\tau_{r}}=\left(1-(-1)^{r} \kappa\right) / 2 \in \mathbb{Z}^{\prime}[G]$, $\mathbb{Z}^{\prime} \otimes H_{\tau, \text { tor }}^{0}=H^{0}\left(G_{K}, \mathbb{Q} / \mathbb{Z}^{\prime}(r)\right)$ and $\mathcal{L}_{\tau_{r}}=L_{K / k, S}(r)^{\#}$. In this case it can be shown that if $\operatorname{ETNC}\left(\mathbb{Q}(r)_{K}, \mathbb{Z}[G]\right)$ is valid, then the equality of Theorem 8.2.1(iii) both refines and generalizes the inclusion conjectured by Coates and Sinnott in $[36$, Conj. 1]. For further details in this regard, and also a discussion of connections to other related work, see $[26, \S 5]$.

\section{Remark 8.2.5. Elliptic curves}

In the setting of Proposition 4.3.1, the module

$$
\left(H_{\tau_{E, \Lambda}}^{0}\right)_{\text {tor }}=\left(\Lambda \otimes E(K)_{\text {tor }}\right) \oplus\left(\Lambda \otimes\left(E(K)_{\text {tor }}\right)^{\vee}\right) \oplus c_{E, \Lambda}^{0}(K)
$$

has order prime to $|G|$ and so Theorem 8.2.1(iii) can be applied. Thus, since $\operatorname{Fit}_{\Lambda[G]}\left(\Lambda \otimes E(K)_{\text {tor }}\right)=\operatorname{Fit}_{\Lambda[G]}\left(\Lambda \otimes\left(E(K)_{\text {tor }}\right)^{\vee}\right)$ (as can be seen by applying $[26$, Lem. 6]), we deduce that if $\amalg\left(E_{/ K}\right)$ is finite, then the conjectural vanishing of $\chi\left(\tau_{E, \Lambda}\right)$ implies that

$$
\operatorname{Fit}_{\Lambda[G]}\left(\Lambda \otimes E(K)_{\text {tor }}\right)^{2} \frac{\operatorname{Fit}_{\Lambda[G]}\left(c_{E, \Lambda}^{0}(K)\right)}{\operatorname{Fit}_{\Lambda[G]}\left(c_{E, \Lambda}^{1}(K)\right)} \mathcal{L}_{\tau_{E, \Lambda}}=\operatorname{Fit}_{\Lambda[G]}\left(\Lambda \otimes \operatorname{Sel}\left(E_{/ K}\right)\right)
$$


where $\left(\mathcal{L}_{\tau_{E, \Lambda}}\right)_{\psi}=\Omega(E)^{-\psi(1)} \tau^{*}(\psi) L_{S_{\mathrm{ram}}}(E, \bar{\psi}, 1)$ for all $\psi \in \operatorname{Irr}(G)$. This (conjectural) equality is a 'strong main conjecture' of the kind that Mazur and Tate ask for in [73, Remark after Conj. 3]. It would be interesting to know the precise relation between the above formula and the explicit conjectural formulas for the Fitting ideals of Selmer groups that are formulated by Kurihara in [68].

\section{Remark 8.2.6. $\mathbb{Q}(1)_{K}$}

If $\tau$ is equal to $\tau_{r}$ with $r \leq 0$ as in Remark 8.2.4, then $\Upsilon_{\tau}$ is equal to the subset of $\operatorname{Irr}(G)$ comprising those characters $\psi$ at which $\left(\mathcal{L}_{\tau}^{*}\right)_{\psi}^{\#}$ is equal to the value at $s=r$ of the appropriate Artin $L$-function. If $\tau=\tau_{E, \Lambda}$ as in Remark 8.2.5, then a similar description of $\Upsilon_{\tau}$ would follow as a consequence of the validity of [23, Conj. 4(ii)]. However, if $\tau$ is equal to $\tau_{1}$ as in Remark 8.2.4 then, unless $K=k=\mathbb{Q}$, $\Upsilon_{\tau}$ is not equal to the subset of $\operatorname{Irr}(G)$ comprising those characters $\psi$ at which $\left(\mathcal{L}_{\tau}^{*}\right)_{\psi}^{\#}$ is equal to a multiple of the value at $s=1$ of the Artin $L$-function and this accounts for the fact that the formula of Theorem 8.2.1(iii) is trivial in this case. Nevertheless, in this case a more interesting variant of Theorem 8.2.1(iii) arises if one replaces $e_{\tau_{1}}$ by a different idempotent. Indeed, in his recent thesis [62] Andrew Jones has shown that the image under multiplication by $e_{-}$of the equality of Theorem 8.2.1(i) with $\tau=\tau_{1}$ implies a natural refinement of the 'integrality conjecture' that is formulated by Solomon in [88].

\section{Remark 8.2.7. Generalisations}

In his recent thesis [81], Andrew Parker has proved generalisations of Theorem 8.2.1 (and of the explicit examples discussed in Remarks 8.2.4 and 8.2.5) in both of the following contexts.

- Gorenstein rings. If $\mathfrak{A}:=\Lambda[G] e_{\tau}$ is isomorphic to $\operatorname{Hom}_{\Lambda}(\mathfrak{A}, \Lambda)$ as an $\mathfrak{A}$-module, then there is an analogue of Theorem 8.2.1(iii) even if the $\Lambda[G]$-module $\left(H_{\tau, \Lambda}^{0}\right)_{\text {tor }}$ is not perfect.

- Non-abelian extensions. If $G$ is non-abelian (but $\left(H_{\tau, \Lambda}^{0}\right)$ tor is still perfect), then there is an analogue of Theorem 8.2.1(iii) that involves a natural notion of Fitting invariant for modules over non-commutative rings.

8.3. The proof of Theorem 5.2.1(iv)(c). We now use the notation and hypotheses of Theorem 5.2.1(iv)(c). 
Under the stated hypotheses the $\Lambda[G]$-modules $H_{\text {tor }}^{0}$ and $H_{\text {tor }}^{1}$ are finite and of projective dimension at most one and the $\Lambda[G]$-modules $H_{\mathrm{tf}}^{0}$ and $H_{\mathrm{tf}}^{1}$ are locallyfree of rank $d$. Thus, if $\Psi^{\bullet}$ is any complex as in (18), then [67, Rem. b) following Th. 2] induces a canonical isomorphism

$$
\operatorname{Det}_{\Lambda[G]}\left(\Psi^{\bullet}\right) \cong \operatorname{Det}_{\Lambda[G]}\left(H^{0}\right) \otimes_{\mathcal{P}(\Lambda[G])} \operatorname{Det}_{\Lambda[G]}\left(H^{1}\right)^{-1}
$$

whilst (24) implies that for $i=0,1$ one has

$$
\operatorname{Det}_{\Lambda[G]}\left(H^{i}\right)=\operatorname{Fitt}_{\Lambda[G]}\left(H_{\mathrm{tor}}^{i}\right)^{-1}\left(\wedge_{\Lambda[G]}^{d} H_{\mathrm{tf}}^{i}, d\right)
$$

in $\operatorname{Det}_{\mathbb{Q}[G]}\left(\mathbb{Q} \otimes \Lambda H^{i}\right)=\left(\mathbb{Q} \otimes_{\Lambda} \wedge_{\Lambda[G]}^{d} H^{i}, d\right)$. In this case the equality of Theorem 8.2.1(i) is thus equivalent to an equality of the form

$$
\wedge_{\mathbb{R}[G]}^{d} \lambda_{\tau}\left(\operatorname{Fitt}_{\Lambda[G]}\left(H_{\mathrm{tor}}^{1}\right) \wedge_{\Lambda[G]}^{d} H_{\mathrm{tf}}^{0}\right)=\mathcal{L}_{\tau}^{*} \cdot \operatorname{Fitt}_{\Lambda[G]}\left(H_{\mathrm{tor}}^{0}\right) \wedge_{\Lambda[G]}^{d} H_{\mathrm{tf}}^{1} .
$$

Now the $\Lambda[G]$-module $\operatorname{Fitt}_{\Lambda[G]}\left(H_{\text {tor }}^{0}\right) \wedge_{\Lambda[G]}^{d} H_{\mathrm{tf}}^{1}$ is locally-free of rank one (indeed, the second factor is obviously locally-free of rank one, whilst the first factor is an invertible ideal of $\Lambda[G]$ since the projective dimension of $H_{\text {tor }}^{0}$ is at most one). In particular, the last displayed equality shows that the $\mathbb{Z}_{(p)}[G]$-submodule $\mathcal{E}_{p}:=\mathbb{Z}_{(p)} \otimes_{\Lambda} \operatorname{Fitt}_{\Lambda[G]}\left(H_{\mathrm{tor}}^{1}\right) \wedge_{\Lambda[G]}^{d} H_{\mathrm{tf}}^{0}$ of $\mathbb{Z}_{(p)} \otimes_{\Lambda} \wedge_{\Lambda[G]}^{d} H_{\mathrm{tf}}^{0}$ is both free of rank one and such that

$$
\wedge_{\mathbb{R}[G]}^{d} \lambda_{\tau}\left(\mathcal{E}_{p}\right)=\mathbb{Z}_{(p)} \otimes_{\Lambda} \mathcal{L}_{\tau}^{*} \cdot \operatorname{Fitt}_{\Lambda[G]}\left(H_{\text {tor }}^{0}\right) \wedge_{\Lambda[G]}^{d} H_{\mathrm{tf}}^{1}
$$

For this choice of $\mathcal{E}_{p}$ it is also clear that there is an isomorphism of finite $\mathbb{Z}_{(p)}[G]$ modules $\left(\wedge_{\mathbb{Z}_{(p)}[G]}^{d}\left(\mathbb{Z}_{(p)} \otimes_{\Lambda} H_{\mathrm{tf}}^{0}\right)\right) / \mathcal{E}_{p} \cong \mathbb{Z}_{(p)} \otimes_{\Lambda} \Lambda[G] /$ Fitt $_{\Lambda[G]}\left(H_{\text {tor }}^{1}\right)$ and hence an equality

$$
\begin{aligned}
& \operatorname{Ann}_{\mathbb{Z}_{(p)}[G]}\left(\left(\wedge_{\mathbb{Z}_{(p)}[G]}^{d}\left(\mathbb{Z}_{(p)} \otimes_{\Lambda} H_{\mathrm{tf}}^{0}\right)\right) / \mathcal{E}_{p}\right) \\
= & \mathbb{Z}_{(p)} \otimes_{\Lambda} \operatorname{Ann}_{\Lambda[G]}\left(\Lambda[G] / \operatorname{Fitt}_{\Lambda[G]}\left(H_{\text {tor }}^{1}\right)\right) \\
= & \mathbb{Z}_{(p)} \otimes_{\Lambda} \operatorname{Fitt}_{\Lambda[G]}\left(H_{\text {tor }}^{1}\right) .
\end{aligned}
$$

Thus, to complete the proof of Theorem 5.2.1(iv)(c), and hence also of Theorem 5.2.1 itself, it only remains to choose a free rank one $\Lambda[G]$-submodule $\mathcal{E}$ of $\operatorname{Fitt}_{\Lambda[G]}\left(H_{\text {tor }}^{1}\right) \wedge_{\Lambda[G]}^{d} H_{\text {tf }}^{0}$ for which one has $\mathbb{Z}_{(p)} \otimes_{\Lambda} \mathcal{E}=\mathcal{E}_{p}$. Indeed, such a module $\mathcal{E}$ exists as a consequence of Roiter's Lemma [37, (31.6)] and the fact that $\operatorname{Fitt}_{\Lambda[G]}\left(H_{\text {tor }}^{1}\right) \wedge_{\Lambda[G]}^{d} H_{\mathrm{tf}}^{0}$ is a locally-free $\Lambda[G]$-module of rank one. 


\section{EXPLICIT CONGRUENCES FOR THE DEGREE ZERO COMPONENT}

In this section we prove Theorem 5.1.1(v) and then discuss arithmetic applications in the setting of the examples discussed in $\S 4$. The argument used in this section is a natural abstraction of that used in [17].

We assume that the $\Lambda[G]$-module $\left(H_{\tau}^{0}\right)_{\text {tor }}$ is perfect (cf. Remark 8.2.3) and set $n_{\tau}:=\operatorname{dim}_{\mathbb{Q}}\left(\mathbb{Q} \otimes_{\Lambda} H_{\tau, G}^{1}\right)$. Then the composite surjection $H_{\tau}^{1} \rightarrow H_{\tau, G}^{1} \rightarrow\left(H_{\tau, G}^{1}\right)_{\mathrm{tf}} \cong$ $\Lambda^{n_{\tau}} \operatorname{implies}_{\operatorname{Fit}_{\Lambda[G]}}\left(H_{\tau}^{1}\right) \subseteq \operatorname{Fit}_{\Lambda[G]}\left(\Lambda^{n_{\tau}}\right)=\operatorname{Fit}_{\Lambda[G]}(\Lambda)^{n_{\tau}}$. But $\operatorname{Fit}_{\Lambda[G]}(\Lambda)$ is equal to the kernel $I_{G, \Lambda}$ of the homomorphism of $\Lambda[G]$-modules $\epsilon: \Lambda[G] \rightarrow \Lambda$ with $\epsilon(g)=1$ for each $g$ in $G$. Hence, from Theorem 8.2.1(iii), one has

$$
\operatorname{Fit}_{\Lambda[G]}\left(\left(H_{\tau}^{0}\right)_{\text {tor }}\right) \cdot \mathcal{L}_{\tau} \subseteq I_{G, \Lambda}^{n_{\tau}} .
$$

We note in passing that this inclusion provides an explicit bound on the denominator of the element $\mathcal{L}_{\tau}$ of $\mathbb{Q}[G]$.

For the rest of this section we fix an element $\alpha$ of $\operatorname{Fit}_{\Lambda[G]}\left(\left(H_{\tau}^{0}\right)_{\text {tor }}\right)$ and describe the image of $\alpha \cdot \mathcal{L}_{\tau}$ under the projection $I_{G, \Lambda}^{n_{\tau}} \rightarrow I_{G, \Lambda}^{n_{\tau}} / I_{G, \Lambda}^{n_{\tau}+1}$ in terms of the discriminant of a natural algebraic height pairing.

9.1. $G$-valued height pairings. The pairing we define here is motivated by the formalism of height pairings developed by Nekovář in [78, §11].

At the outset we fix a bounded complex of perfect $\Lambda[G]$-modules $C^{\bullet}$ and set $C_{G}^{\bullet}:=\Lambda \otimes_{\Lambda[G]}^{\mathbb{L}} C^{\bullet}$ and $C^{\bullet}, G:=\operatorname{RHom}_{\Lambda[G]}\left(\Lambda, C^{\bullet}\right)$. Then, since each term of $C^{\bullet}$ is perfect, there is a natural isomorphism in $\mathcal{D}(\Lambda)$ between $C_{G}^{\bullet}$, resp. $C^{\bullet}, G$, and the complex which in each degree $i$ is equal to $\left(C^{i}\right)_{G}$, resp. $\left(C^{i}\right)^{G}$, and in which the differentials are induced by those of $C^{\bullet}$. For this reason, the action of $\operatorname{Tr}_{G}:=\sum_{g \in G} g \in \mathbb{Z}[G]$ on each module $C^{i}$ induces an isomorphism in $D(\Lambda)$ from $C_{G}^{\bullet}$ to $C^{\bullet}, G$.

Taken in conjunction with the isomorphism $C_{G}^{\bullet} \cong C^{\bullet}, G$ described above, the tautological exact sequence $0 \rightarrow I_{G, \Lambda} \rightarrow \Lambda[G] \rightarrow \Lambda \rightarrow 0$ induces an exact triangle in $D(\Lambda[G])$ of the form

$$
I_{G, \Lambda} \otimes_{\Lambda[G]}^{\mathbb{L}} C^{\bullet} \rightarrow C^{\bullet} \rightarrow C^{\bullet, G} \rightarrow I_{G, \Lambda} \otimes_{\Lambda[G]}^{\mathbb{L}} C^{\bullet}[1]
$$

If now $C^{\bullet}$ is also acyclic outside degrees 0 and 1 , then there are natural identifications $H^{0}\left(C^{\bullet}, G\right)=H^{0}\left(C^{\bullet}\right)^{G}, H^{1}\left(I_{G, \Lambda} \otimes_{\Lambda[G]}^{\mathbb{L}} C^{\bullet}\right) \cong I_{G, \Lambda} \otimes_{\Lambda[G]} H^{1}\left(C^{\bullet}\right)$ and $H^{1}\left(C^{\bullet}\right)_{G} \cong H^{1}\left(C_{G}^{\bullet}\right) \cong H^{1}\left(C^{\bullet}, G\right)$ (where the second isomorphism is induced by 
the isomorphism $C_{G}^{\bullet} \cong C^{\bullet, G}$ described above). In this case the cohomology sequence of (29) therefore induces an exact sequence of $\Lambda[G]$-modules

$$
H^{0}\left(C^{\bullet}\right) \stackrel{\operatorname{Tr}_{G}}{\longrightarrow} H^{0}\left(C^{\bullet}, G\right) \rightarrow I_{G, \Lambda} \otimes_{\Lambda[G]} H^{1}\left(C^{\bullet}\right)
$$

as well as a $G$-equivariant 'Bockstein homomorphism'

$$
\beta_{C}, G: H^{0}\left(C^{\bullet}\right)^{G}=H^{0}\left(C^{\bullet, G}\right) \rightarrow I_{G, \Lambda} \otimes_{\Lambda[G]} H^{1}\left(C^{\bullet}\right) \rightarrow I_{G, \Lambda} / I_{G, \Lambda}^{2} \otimes_{\Lambda} H^{1}\left(C^{\bullet, G}\right),
$$

where the last arrow is induced by passing to $G$-coinvariants and then identifying $H^{1}\left(C^{\bullet}\right)_{G}$ with $H^{1}\left(C^{\bullet}, G\right)$ in the manner described above. We set $H^{1}\left(C^{\bullet}, G\right)^{*}:=$ $\operatorname{Hom}_{\Lambda}\left(H^{1}\left(C^{\bullet}, G\right), \Lambda\right)=\operatorname{Hom}_{\Lambda}\left(H^{1}\left(C^{\bullet}, G\right)_{\mathrm{tf}}, \Lambda\right)$ and write

$$
\rho_{C}, G: H^{0}\left(C^{\bullet}\right)^{G} \times H^{1}\left(C^{\bullet, G}\right)^{*} \rightarrow I_{G, \Lambda} / I_{G, \Lambda}^{2},
$$

for the pairing induced by $\beta_{C} \bullet G$. Then, in keeping with the philosophy of Mazur and Tate in [73] and of Gross in [55], the canonical isomorphism $I_{G, \Lambda} / I_{G, \Lambda}^{2} \cong \Lambda \otimes G$ allows one to regard $\rho_{C}, G$ as taking values in the group $\Lambda \otimes G \subseteq G$.

9.2. Statement of the main result. If $\Psi^{\bullet}$ is any complex as in (18), then the assumed containment $\chi(\tau) \in K_{0}(\Lambda[G], \mathbb{Q}[G])$ implies that $\operatorname{Det}_{\mathbb{R}[G]} \lambda_{\tau}$ restricts to give an isomorphism in $\mathcal{P}(\mathbb{Q}[G])$

$$
\operatorname{Det}_{\mathbb{Q}[G]}\left(H^{0}\left(\Psi^{\bullet}\right) \otimes_{\Lambda} \mathbb{Q}\right) \stackrel{\sim}{\longrightarrow} \mathcal{L}_{\tau}^{*} \cdot \operatorname{Det}_{\mathbb{Q}[G]}\left(H^{1}\left(\Psi^{\bullet}\right) \otimes_{\Lambda} \mathbb{Q}\right),
$$

and hence also (after multiplying by $\operatorname{Tr}_{G}$ ) an isomorphism of $\mathbb{Q}$-lines

$$
\wedge_{\mathbb{R}}^{n_{\tau}} \lambda_{\tau}^{G}: \wedge_{\mathbb{Q}}^{n_{\tau}}\left(H^{0}\left(\Psi^{\bullet}\right)^{G} \otimes_{\Lambda} \mathbb{Q}\right) \stackrel{\sim}{\longrightarrow} \mathcal{L}_{\tau}^{*} \cdot \wedge_{\mathbb{Q}}^{n_{\tau}}\left(H^{1}\left(\Psi^{\bullet}, G\right) \otimes_{\Lambda} \mathbb{Q}\right)
$$

In particular, after fixing ordered $\Lambda$-bases $x$.: $=\left\{x_{i}: 1 \leq i \leq n_{\tau}\right\}$ and $y$.:= $\left\{y_{j}: 1 \leq j \leq n_{\tau}\right\}$ of $\left(H^{0}\left(\Psi^{\bullet}\right)^{G}\right)_{\mathrm{tf}}$ and $H^{1}\left(\Psi^{\bullet}, G\right)_{\mathrm{tf}}$ respectively, we may define a rational number $r_{\alpha, x, y}$. by means of the equality

$$
\alpha \mathcal{L}_{\tau}^{*} \cdot \wedge_{1 \leq i \leq n_{\tau}} y_{i}=r_{\alpha, x, y .} \cdot\left(\wedge_{\mathbb{R}}^{n_{\tau}} \lambda_{\tau}^{G}\right)\left(\wedge_{1 \leq j \leq n_{\tau}} x_{j}\right) \in \wedge_{\mathbb{R}}^{n_{\tau}}\left(H^{1}\left(\Psi^{\bullet, G}\right) \otimes_{\Lambda} \mathbb{R}\right) .
$$

We then define a subring of $\mathbb{Q}$ by setting

$$
\Lambda_{\alpha}:=\Lambda\left[r_{\alpha, x, y .}\right]
$$

(this subring is indeed independent of the choice of ordered bases $x$. and $y$.) and a 'discriminant element' by setting

$$
\operatorname{disc}_{\alpha}(\tau):=r_{\alpha, x ., y} \cdot \operatorname{det}\left(\rho_{\Psi} \bullet, G\left(x_{i}, y_{j}^{*}\right)_{1 \leq i, j \leq n_{\tau}}\right) \in \Lambda_{\alpha} \otimes_{\Lambda} I_{G, \Lambda}^{n_{\tau}} / I_{G, \Lambda}^{n_{\tau}+1}
$$

where $y_{j}^{*}$ denotes the element of $H^{1}\left(\Psi^{\bullet}, G\right) *$ that is dual to $y_{j}$ (so, for all indices $i$ and $j$, one has $y_{j}^{*}\left(y_{i}\right)=1$ if $i=j$ and $y_{j}^{*}\left(y_{i}\right)=0$ otherwise). 
Lemma 9.2.1. $\operatorname{disc}_{\alpha}(\tau)$ is well-defined and depends only upon $\alpha$ and $\rho_{\tau}$.

Proof. If $\Psi^{\bullet}, x$. and $y$. are fixed, then $\operatorname{det}\left(\rho_{\Psi, G}\left(x_{i}, y_{j}^{*}\right)_{1 \leq i, j \leq n_{\tau}}\right)$ is well-defined because $\left(H_{\tau}^{0}\right)_{\text {tor }}$ is $\Lambda[G]$-perfect. Indeed, this combines with the exactness of (30) (with $C^{\bullet}=\Psi^{\bullet}$ ) to imply that $\left(H^{0}\left(\Psi^{\bullet}\right)^{G}\right)_{\text {tor }}=\left(\left(H_{\tau}^{0}\right)_{\text {tor }}\right)^{G}=\operatorname{Tr}_{G}\left(\left(H_{\tau}^{0}\right)_{\text {tor }}\right) \subseteq$ $\operatorname{ker}\left(\beta_{\Psi} \bullet, G\right)$.

We must show that $\operatorname{disc}_{\alpha}(\tau)$ is independent of the choices of $\Psi^{\bullet}, x$. and $y$. But if $\Psi^{\bullet}$ is fixed (as in (18)) then the definition of $r_{\alpha, x . y}$. combines with standard formulas for the change in discriminant resulting from a change of bases to ensure that $\operatorname{disc}_{\alpha}(\tau)$ is independent of the choices of ordered bases $x$. and $y$. On the other hand, if $\tilde{\Psi}^{\bullet}$ is any other choice of complex as in (18), then there is an isomorphism $\iota: \tilde{\Psi}^{\bullet} \cong \Psi^{\bullet}$ in $D^{\text {perf }}(\Lambda[G])$ such that $H^{i}(\iota)$ is the identity map for $i=0,1$. The naturality with respect to $\iota$ of the construction of Bockstein homomorphisms thus implies that $\rho_{\tilde{\Psi} \bullet, G}=\rho_{\Psi \bullet, G}$, as required.

We can now state the main result of this section.

Theorem 9.2.2. If $\alpha$ is any element of $\operatorname{Fit}_{\Lambda[G]}\left(\left(H_{\tau}^{0}\right)_{\text {tor }}\right)$, then the image of $\alpha \cdot \mathcal{L}_{\tau}$ in $\Lambda_{\alpha} \otimes_{\Lambda} I_{G, \Lambda}^{n_{\tau}}$ satisfies $\alpha \cdot \mathcal{L}_{\tau} \equiv \operatorname{disc}_{\alpha}(\tau)\left(\bmod \Lambda_{\alpha} \otimes_{\Lambda} I_{G, \Lambda}^{n_{\tau}+1}\right)$.

This result is clear if $n_{\tau}=0$. Indeed, in this case the augmentation map $\epsilon: \Lambda[G] \rightarrow \Lambda$ induces an isomorphism $I_{G, \Lambda}^{n_{\tau}} / I_{G, \Lambda}^{n_{\tau}+1}=\Lambda[G] / I_{G, \Lambda} \cong \Lambda$ and both $\operatorname{disc}_{\alpha}(\tau)=r_{\alpha, x, y}=\epsilon\left(\alpha \cdot \mathcal{L}_{\tau}^{*}\right)$ and $\epsilon\left(e_{\tau}\right)=1$ and so the stated congruence is equivalent to the obvious equality $\epsilon\left(\alpha \cdot \mathcal{L}_{\tau}\right)=\epsilon\left(\alpha \cdot \mathcal{L}_{\tau}^{*}\right)$.

In the remainder of the argument we therefore assume (and without further explicit comment) that $n_{\tau}>0$. In particular, since in this case $I_{G, \Lambda}^{n_{\tau}} / I_{G, \Lambda}^{n_{\tau}+1}$ is a quotient of the $n_{\tau}$-th symmetric power of $\Lambda \otimes G$, it is a finite group of order dividing a power of $|G|$. The argument we use in this case is closely modelled on that given in $[17, \S 5.3-5]$.

9.3. An explicit version of Theorem 8.2.1(i). We set $n:=n_{\tau}$ and for any natural number $m$ let $|m|$ denote the set of integers $i$ with $1 \leq i \leq m$. We also set $T:=\left(H_{\tau}^{0}\right)_{\text {tor }}$.

Lemma 9.3.1. There exists a complex $\Psi^{\bullet}$ as in (18) and an endomorphism $\phi$ of a finitely generated free $\Lambda[G]$-module $F$ which satisfy both of the following conditions. 
Let $F^{\bullet}$ denote the complex $F \stackrel{\phi}{\rightarrow} F$, where the first term is placed in degree 0 .

(i) There is a short exact sequence of perfect complexes of $\Lambda[G]$-modules

$$
0 \rightarrow F^{\bullet} \stackrel{\varrho}{\rightarrow} \Psi^{\bullet} \rightarrow Q[0] \rightarrow 0
$$

where $Q$ is a finite cohomologically-trivial $\Lambda[G]$-module which contains $T$ and is such that $|Q / T|$ is coprime to $|G|$.

(ii) There exists an integer $n^{\prime}$ with $n^{\prime} \geq n$ and an ordered $\Lambda[G]$-basis $\left\{b_{i}: i \in\right.$ $\left.\left|n^{\prime}\right|\right\}$ of $F$ which satisfies both of the following conditions.

(a) The $\Lambda[G]$-module $F_{1}$ generated by $\left\{b_{i}: i \in|n|\right\}$ satisfies $F_{1}^{G}=$ $\operatorname{ker}\left(\phi^{G}\right)$ and, for each $i \in|n|, \operatorname{Tr}_{G}\left(b_{i}\right)$ is a pre-image of $y_{i}$ under the composite homomorphism

$$
F_{1}^{G} \subseteq F^{G} \rightarrow \operatorname{cok}\left(\phi^{G}\right) \rightarrow H^{1}\left(\Psi^{\bullet, G}\right)_{\mathrm{tf}},
$$

where the second map is tautological and the third is induced by $H^{1}\left(R \operatorname{Hom}_{\Lambda[G]}(\Lambda, \varrho)\right)$.

(b) The $\Lambda[G]$-module $F_{2}$ generated by $\left\{b_{i}: n<i \leq n^{\prime}\right\}$ satisfies $\phi^{G}\left(F_{2}^{G}\right) \subseteq$ $F_{2}^{G}$.

Proof. We first fix a complex $\Psi^{\bullet}$ as in (18) for which $\Psi^{1}$ is a finitely generated free $\Lambda[G]$-module (this is always possible) and set $F:=\Psi^{1}$. Then, since $T$ is a perfect $\Lambda[G]$-module, $P:=\Psi^{0} / T$ is a finitely generated $\Lambda[G]$-module which is both cohomologically-trivial as a $G$-module and torsion-free as a $\Lambda$-module. Any such $\Lambda[G]$-module $P$ is projective $[3$, Th. 8]. In addition, the $\mathbb{Q}[G]$-modules $\mathbb{Q} \otimes_{\Lambda} H^{0}\left(\Psi^{\bullet}\right)$ and $\mathbb{Q} \otimes_{\Lambda} H^{1}\left(\Psi^{\bullet}\right)$ are isomorphic and so, just as in the proof of Lemma 3.2.1(ii), we may deduce from Swan's Theorem that for each prime $q$ in $\operatorname{Spec}(\Lambda)$ the $\mathbb{Z}_{q}[G]$-modules $\mathbb{Z}_{q} \otimes_{\Lambda} P$ and $\mathbb{Z}_{q} \otimes_{\Lambda} F$ are isomorphic. We may thus apply Roiter's Lemma [37, (31.6)] to deduce that there exists a $\Lambda[G]$-submodule $P^{\prime}$ of $P$ for which the quotient $P / P^{\prime}$ is finite and of order coprime to $|G|$ and there is an isomorphism of $\Lambda[G]$-modules $\iota: F \cong P^{\prime}$. We choose a lift $\tilde{\iota}: F \rightarrow \Psi^{0}$ of $\iota$ through the tautological surjection $\Psi^{0} \rightarrow P$ and then set $\varsigma:=d^{0} \circ \tilde{\iota} \in \operatorname{End}_{\Lambda[G]}(F)$ with $d^{0}$ the differential in degree 0 of $\Psi^{\bullet}$.

The exact sequence of $\Lambda$-modules $0 \rightarrow \operatorname{ker}\left(\varsigma^{G}\right) \rightarrow F^{G} \rightarrow \operatorname{im}\left(\varsigma^{G}\right) \rightarrow 0$ splits and so there is a submodule $D$ of $F^{G}$ which $\varsigma^{G}$ maps bijectively to $\operatorname{im}\left(\varsigma^{G}\right)$. Also, if $N$ is the pre-image of $\operatorname{cok}\left(\varsigma^{G}\right)$ tor under the tautological surjection $F^{G} \rightarrow$ $\operatorname{cok}\left(\varsigma^{G}\right)$, then the exact sequence $0 \rightarrow N \rightarrow F^{G} \rightarrow \operatorname{cok}\left(\varsigma^{G}\right)_{\mathrm{tf}} \rightarrow 0$ splits and so 
there is a submodule $D^{\prime}$ of $F^{G}$ which maps bijectively to $\operatorname{cok}\left({ }^{G}\right)_{\mathrm{tf}}$ under the natural surjection. Now $D^{\prime}$ and $\operatorname{ker}\left(\varsigma^{G}\right)$ have the same $\Lambda$-rank since there are isomorphisms $\mathbb{Q} \otimes_{\Lambda} D^{\prime} \cong \mathbb{Q} \otimes_{\Lambda} \operatorname{cok}\left(\varsigma^{G}\right) \cong \mathbb{Q} \otimes_{\Lambda} \operatorname{cok}(\varsigma)^{G} \cong \mathbb{Q} \otimes_{\Lambda} \operatorname{ker}(\varsigma)^{G} \cong \mathbb{Q} \otimes_{\Lambda}$ $\operatorname{ker}\left(\varsigma^{G}\right)$. The direct sum decompositions $\operatorname{ker}\left(\varsigma^{G}\right) \oplus D=F^{G}=N \oplus D^{\prime}$ therefore imply that there exists an automorphism $\psi^{\prime}$ of $F^{G}$ with both $\psi^{\prime}(N)=D$ and $\psi^{\prime}\left(D^{\prime}\right)=\operatorname{ker}\left(\varsigma^{G}\right)$. Then one has $\psi^{\prime} \circ \varsigma^{G}(D) \subseteq D$ and $\operatorname{ker}\left(\psi^{\prime} \circ \varsigma^{G}\right)=\operatorname{ker}\left(\varsigma^{G}\right)$ maps bijectively to $\operatorname{cok}\left(\psi^{\prime} \circ \varsigma^{G}\right)_{\mathrm{tf}}$ under the tautological surjection $F^{G} \rightarrow \operatorname{cok}\left(\psi^{\prime} \circ \varsigma^{G}\right)_{\mathrm{tf}}$.

Since $F$ is a free $\Lambda[G]$-module we may choose $\tilde{\psi}$ in $\operatorname{Aut}_{\Lambda[G]}(F)$ with $\tilde{\psi}^{G}=\psi^{\prime}$. We set $\phi:=\tilde{\psi} \circ \varsigma \in \operatorname{End}_{\Lambda[G]}(F)$ and let $\varrho$ denote the morphism of complexes from the complex $F^{\bullet}$ described in the statement of Lemma 9.3.1 to $\Psi^{\bullet}$ that is equal to $\tilde{\iota}$ in degree 0 and to $\tilde{\psi}^{-1}$ in degree 1 . It is easily checked that this gives rise to a short exact sequence as in claim (i) in which $Q / T \cong P / P^{\prime}$.

Now $\phi^{G}=\psi^{\prime} \circ \varsigma^{G}$ and so the above remarks imply both that $\phi^{G}(D) \subseteq D$ and that the natural map $\operatorname{ker}\left(\phi^{G}\right) \rightarrow \operatorname{cok}\left(\phi^{G}\right)_{\mathrm{tf}}$ is bijective. We next observe that the decomposition $F^{G}=\operatorname{ker}\left(\phi^{G}\right) \oplus D$ lifts to a direct sum decomposition $F=F_{1} \oplus F_{2}$ of $\Lambda[G]$-modules in which both $F_{1}$ and $F_{2}$ are free (of ranks $n$ and $n^{\prime}-n$ respectively), $F_{1}^{G}=\operatorname{ker}\left(\phi^{G}\right)$ and $F_{2}^{G}=D$. We write $\kappa$ for the displayed composite homomorphism in claim (ii)(a). Then the above observations imply $\kappa$ is bijective and so $\left\{\kappa^{-1}\left(y_{i}\right): i \in|n|\right\}$ is a $\Lambda$-basis of $F_{1}^{G}=\operatorname{Tr}_{G}\left(F_{1}\right)$. It is then easily shown that there exists a $\Lambda[G]$-basis $\left\{b_{i}: i \in|n|\right\}$ of $F_{1}$ such that $\operatorname{Tr}_{G}\left(b_{i}\right)=\kappa^{-1}\left(y_{i}\right)$ for each $i \in|n|$. To complete the proof of claim (ii) we then simply let $\left\{b_{i}: n<i \leq n^{\prime}\right\}$ be any choice of (ordered) $\Lambda[G]$-basis of $F_{2}$.

For any $\Lambda$-module $X$, resp. homomorphism of $\Lambda$-modules $\kappa$, we set $X_{\mathbb{R}}:=$ $\mathbb{R} \otimes_{\Lambda} X$ and $\kappa_{\mathbb{R}}:=\mathbb{R} \otimes_{\Lambda} \kappa$. Then, by applying $\operatorname{Det}_{\Lambda[G]}$ to the exact sequence in Lemma 9.3.1(i), one finds that

$$
\begin{aligned}
\vartheta_{\lambda_{\tau}}\left(\operatorname{Det}_{\Lambda[G]} \Psi^{\bullet}\right) & =\operatorname{Fit}_{\Lambda[G]}(Q)^{-1} \vartheta_{\lambda_{\tau}}\left(\operatorname{Det}_{\Lambda[G]} F^{\bullet}\right) \\
& =\operatorname{Fit}_{\Lambda[G]}(T)^{-1} \operatorname{Fit}_{\Lambda[G]}((Q / T))^{-1} \vartheta_{\lambda_{\tau} \frac{\rho}{\tau}}\left(\operatorname{Det}_{\Lambda[G]} F^{\bullet}\right)
\end{aligned}
$$

where $\lambda_{\tau}^{\varrho}$ denotes the composite isomorphism

$$
H^{1}(\varrho)_{\mathbb{R}}^{-1} \circ \lambda_{\tau} \circ H^{0}(\varrho)_{\mathbb{R}}: H^{0}\left(F^{\bullet}\right)_{\mathbb{R}} \cong H^{1}\left(F^{\bullet}\right)_{\mathbb{R}}
$$


On the other hand, an explicit computation (or [17, Lem. A1]) shows that

$$
\vartheta_{\lambda_{\tau}^{\varrho}}\left(\operatorname{Det}_{\Lambda[G]} F^{\bullet}\right)=\operatorname{det}_{\mathbb{R}[G]}\left(\left\langle\lambda_{\tau}^{\varrho}, \phi\right\rangle_{\iota_{1}, \iota_{2}}\right) \cdot \Lambda[G]
$$

where $\iota_{1}$ and $\iota_{2}$ are a choice of $\mathbb{R}[G]$-equivariant sections to the tautological surjections $F_{\mathbb{R}} \rightarrow \operatorname{im}(\phi)_{\mathbb{R}}$ and $F_{\mathbb{R}} \rightarrow \operatorname{cok}(\phi)_{\mathbb{R}}$ and $\left\langle\lambda_{\tau}^{\varrho}, \phi\right\rangle_{\iota_{1}, \iota_{2}}$ is the (unique) element of $\operatorname{Aut}_{\mathbb{R}[G]}\left(F_{\mathbb{R}}\right)$ that is equal to $\iota_{2} \circ \lambda_{\tau}^{\varrho}$ on $\operatorname{ker}(\phi)_{\mathbb{R}}$ and to $\phi_{\mathbb{R}}$ on $\iota_{1}\left(\operatorname{im}(\phi)_{\mathbb{R}}\right)$. The assumed equality of Theorem 8.2.1(i) therefore implies the existence of an element $c_{\alpha}$ of $\mathbb{Q}[G]^{\times}$with both

$$
\alpha \mathcal{L}_{\tau}^{*}=c_{\alpha} \cdot \operatorname{det}_{\mathbb{R}[G]}\left(\left\langle\lambda_{\tau}^{\varrho}, \phi\right\rangle_{\iota_{1}, \iota_{2}}\right) \in \mathbb{R}[G]^{\times}
$$

and

$$
\Lambda[G] \cdot c_{\alpha}=\alpha \operatorname{Fit}_{\Lambda[G]}(T)^{-1} \operatorname{Fit}_{\Lambda[G]}((Q / T))^{-1} \subseteq \operatorname{Fit}_{\Lambda[G]}((Q / T))^{-1}
$$

(where the last inclusion is valid because $\alpha$ belongs to $\operatorname{Fit}_{\Lambda[G]}(T)$ ).

9.4. The $e_{\tau}$-component. Since the $\mathbb{C}[G]$-module $\mathbb{C} \otimes_{\Lambda} \operatorname{ker}(\phi)$ is isomorphic (via $\left.\mathbb{C} \otimes_{\Lambda} H^{0}(\varsigma)\right)$ to $\mathbb{C} \otimes_{\Lambda} H^{0}\left(\Psi^{\bullet}\right)=\mathbb{C} \otimes_{\Lambda} H_{\tau}^{0}$ the definition of $e_{\tau}$ at the beginning of $\S 8.2$ ensures that for each $\psi \in \operatorname{Irr}(G)$ one has $e_{\psi} e_{\tau} \neq 0$ if and only if $e_{\psi}\left(\mathbb{C} \otimes_{\Lambda} \operatorname{ker}(\phi)\right)=$ 0 . It follows that $\left\langle\lambda_{\tau}^{\varrho}, \phi\right\rangle_{\iota_{1}, \iota_{2}}$ agrees with $\phi_{\mathbb{R}}$ on $e_{\tau} F_{\mathbb{R}}$ and that $\operatorname{det}_{\Lambda[G]}(\phi)=$ $e_{\tau} \operatorname{det}_{\Lambda[G]}(\phi)$ and hence that (34) implies

$$
\alpha \cdot \mathcal{L}_{\tau}=\alpha e_{\tau} \cdot \mathcal{L}_{\tau}^{*}=c_{\alpha} \cdot \operatorname{det}_{\mathbb{R}[G]}\left(\left\langle\lambda_{\tau}^{\varrho}, \phi\right\rangle_{\iota_{1}, \iota_{2}} \mid e_{\tau} F_{\mathbb{R}}\right)=c_{\alpha} \cdot \operatorname{det}_{\Lambda[G]}(\phi) .
$$

This equality is key to the proof of Theorem 9.2.2. Indeed, Lemma 9.3.1(ii) implies that the matrix of $\phi$ with respect to the ordered basis $\left\{b_{i}: i \in\left|n^{\prime}\right|\right\}$ of $F$ is a block matrix of the form

$$
\left(\begin{array}{c|c}
A \mid B \\
\hline C \mid D
\end{array}\right)
$$

where $A \in \mathrm{M}_{n}\left(I_{G, \Lambda}\right), D \in \mathrm{M}_{n^{\prime}-n}(\Lambda[G])$ and all entries of both $B$ and $C$ belong to $I_{G, \Lambda}$. But $\alpha \mathcal{L}_{\tau} \in I_{G, \Lambda}^{n}$ (by $\left.(28)\right), \operatorname{det}(A) \in I_{G, \Lambda}^{n}$ and $c_{\alpha}$ acts naturally on $I_{G, \Lambda}^{n} / I_{G, \Lambda}^{n+1}$ (by (35) and the fact that $|Q / T|$ is coprime to $|G|$ ) and so the matrix representation (37) combines with (36) to imply that

$$
\alpha \cdot \mathcal{L}_{\tau} \equiv \epsilon\left(c_{\alpha}\right) \epsilon(\operatorname{det}(D)) \cdot \operatorname{det}(A)\left(\bmod I_{G, \Lambda}^{n+1}\right) .
$$

To compute the term $\epsilon\left(c_{\alpha}\right) \epsilon(\operatorname{det}(D))$ we first multiply (34) by the idempotent $e_{G}:=|G|^{-1} \operatorname{Tr}_{G}$ to get

$$
\alpha \mathcal{L}_{\tau}^{*} \cdot e_{G}=\epsilon\left(c_{\alpha}\right) \operatorname{det}_{\mathbb{R}}\left(\left\langle\lambda_{\tau}^{\varrho}, \phi\right\rangle_{\iota_{1}, \iota_{2}} \mid F_{\mathbb{R}}^{G}\right) \cdot e_{G} .
$$


Now, by making a suitable choice of sections $\iota_{1}$ and $\iota_{2}$ and then computing with respect to the ordered $\Lambda$-basis $\left\{\operatorname{Tr}_{G}\left(b_{i}\right): 1 \leq i \leq n^{\prime}\right\}$ of $F^{G}$, one finds that the restriction of $\left\langle\lambda_{\tau}^{\varrho}, \phi\right\rangle_{\iota_{1}, \iota_{2}}$ to $F_{\mathbb{R}}^{G}$ is represented by a matrix of the form

$$
\left(\begin{array}{c|c}
A^{\prime} & B^{\prime} \\
\hline 0 & \epsilon(D)
\end{array}\right) .
$$

Here $\epsilon(D) \in \mathrm{M}_{n^{\prime}-n}(\Lambda)$ is the matrix with $\epsilon(D)_{i j}=\epsilon\left(D_{i j}\right)$ for all $i$ and $j$ and $A^{\prime} \in \mathrm{M}_{n}(\Lambda)$ is the matrix of the composite isomorphism

$$
\begin{aligned}
\mathbb{R} \otimes_{\Lambda} F_{1}^{G}=\mathbb{R} \otimes_{\Lambda} \operatorname{ker}\left(\phi^{G}\right) & \stackrel{\mathbb{R} \otimes_{\Lambda} H^{0}\left(\varrho^{G}\right)}{\longrightarrow} \mathbb{R} \otimes_{\Lambda} H^{0}\left(\Psi^{\bullet}\right)^{G} \\
& \stackrel{\lambda_{\tau}^{G}}{\longrightarrow} \mathbb{R} \otimes_{\Lambda} H^{1}\left(\Psi^{\bullet}, G\right) \rightarrow \mathbb{R} \otimes_{\Lambda} F_{1}^{G}
\end{aligned}
$$

where we set $\varrho^{G}:=R \operatorname{Hom}_{\Lambda[G]}(\Lambda, \varrho)$ and the last arrow denotes the inverse of the composite isomorphism in Lemma 9.3.1(ii)(a). But $\operatorname{det}\left(A^{\prime}\right)$ is the determinant of the matrix of the map

$$
\wedge_{\mathbb{R}}^{n} \lambda_{\tau}^{G}: \mathbb{R} \otimes_{\Lambda} \wedge_{\Lambda}^{n}\left(H^{0}\left(\varrho^{G}\right)\left(F_{1}^{G}\right)\right) \rightarrow \mathbb{R} \otimes_{\Lambda} \wedge_{\Lambda}^{n} H^{1}\left(\Psi^{\bullet, G}\right),
$$

as computed with respect to the $\mathbb{R}$-bases $\wedge_{j \in|n|} H^{0}\left(\varrho^{G}\right)\left(\operatorname{Tr}_{G}\left(b_{j}\right)\right)$ and $\wedge_{i \in|n|} y_{i}$. Thus (39) implies that

$$
\begin{aligned}
\alpha \mathcal{L}_{\tau}^{*} \cdot \wedge_{i \in|n|} y_{i} & =\alpha \mathcal{L}_{\tau}^{*} e_{G} \cdot \wedge_{i \in|n|} y_{i} \\
& =\epsilon\left(c_{\alpha}\right) \epsilon(\operatorname{det}(D)) \operatorname{det}\left(A^{\prime}\right) \cdot \wedge_{i \in|n|} y_{i} \\
& =\epsilon\left(c_{\alpha}\right) \epsilon(\operatorname{det}(D)) \cdot\left(\wedge_{\mathbb{R}}^{n} \lambda_{\tau}^{G}\right)\left(\wedge_{j \in|n|} H^{0}\left(\varrho^{G}\right)\left(\operatorname{Tr}_{G}\left(b_{j}\right)\right)\right) \\
& =\epsilon\left(c_{\alpha}\right) \epsilon(\operatorname{det}(D)) \operatorname{det}(b) \cdot\left(\wedge_{\mathbb{R}}^{n} \lambda_{\tau}^{G}\right)\left(\wedge_{j \in|n|} x_{j}\right),
\end{aligned}
$$

where the matrix $b:=\left(b_{i j}\right) \in \mathrm{M}_{n}(\Lambda)$ is defined via the equalities

$$
H^{0}\left(\varrho^{G}\right)\left(\operatorname{Tr}_{G}\left(b_{i}\right)\right)=\sum_{j \in|n|} b_{i j} x_{j} \in\left(H^{0}\left(\Psi^{\bullet}\right)^{G}\right)_{\mathrm{tf}} .
$$

Comparing (40) with (31) shows that $r_{\alpha, x . y .}=\epsilon\left(c_{\alpha}\right) \epsilon(\operatorname{det}(D)) \operatorname{det}(b)$ and so

$$
\alpha \cdot \mathcal{L}_{\tau} \equiv r_{\alpha, x, y .} \operatorname{det}(b)^{-1} \operatorname{det}(A)\left(\bmod I_{G, \Lambda}^{n+1}\right)
$$

by (38). 
9.5. Completion of the proof. Given the congruence at the end of the last section our proof of Theorem 9.2.2 will be completed if we can $\operatorname{show}$ that $\operatorname{disc}_{\alpha}(\tau):=$ $r_{\alpha, x, y .} \operatorname{det}\left(\rho_{\Psi \bullet, G}\left(x_{i}, y_{j}^{*}\right)_{1 \leq i, j \leq n}\right)$ is equal to the image of $r_{\alpha, x, y} \operatorname{det}(b)^{-1} \operatorname{det}(A)$ in $\Lambda_{\alpha} \otimes_{\Lambda} I_{G, \Lambda}^{n} / I_{G, \Lambda}^{n+1}$.

As a first step we compute explicitly the pairing $\rho_{F} \bullet G$.

Lemma 9.5.1. With respect to the ordered $\Lambda$-bases $\left\{\operatorname{Tr}_{G}\left(b_{i}\right): i \in|n|\right\}$ and $\left\{\left(H^{1}\left(\varrho^{G}\right)_{\mathbb{R}}^{-1}\left(y_{j}\right)\right)^{*}: j \in|n|\right\}$ of $H^{0}\left(F^{\bullet}\right)^{G}$ and $H^{1}\left(F^{\bullet}, G\right)^{*}$ respectively, the matrix of $\rho_{F}, G$ is equal to the image of $A$ in $\mathrm{M}_{n}\left(I_{G, \Lambda} / I_{G, \Lambda}^{2}\right)$.

Proof. We write $\pi$ and $\pi^{\prime}$ for the tautological surjections $\operatorname{Tr}_{G}(F)=F^{G} \rightarrow$ $\operatorname{cok}\left(\phi^{G}\right)$ and $I_{G, \Lambda} \rightarrow I_{G, \Lambda} / I_{G, \Lambda}^{2}$ respectively. Then $\beta_{F} \bullet, G$ can be computed as the connecting homomorphism which arises when applying the Snake lemma to the following commutative diagram (in which both rows and the third column are exact and the first column is a complex)

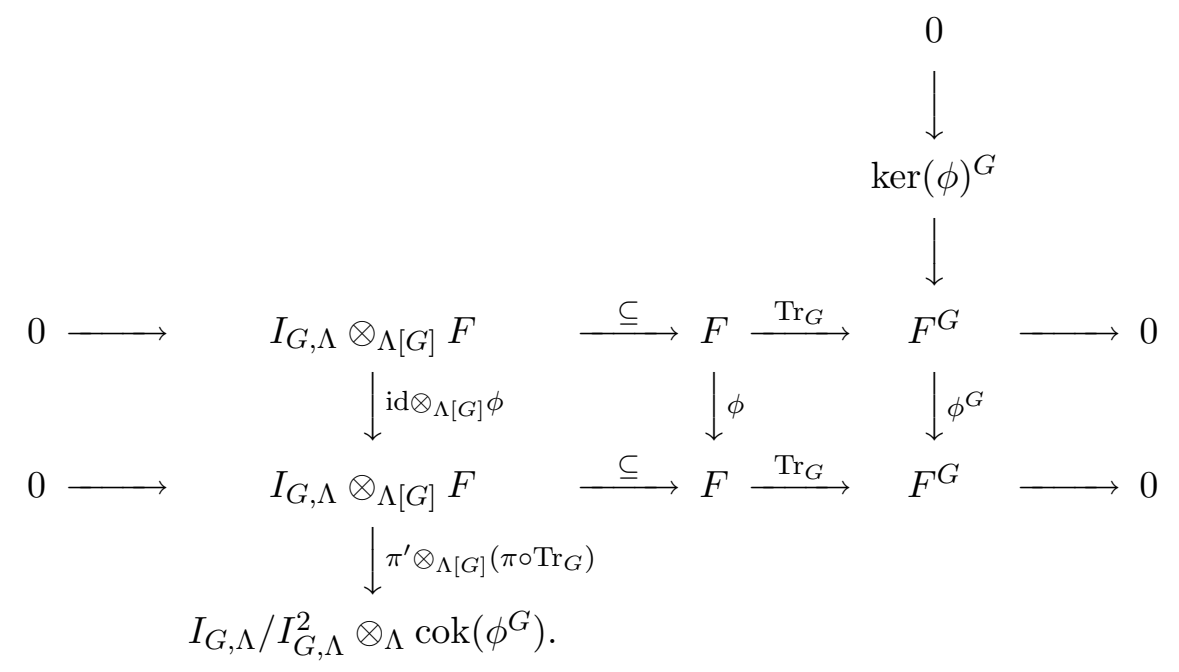

By computing this connecting homomorphism using the matrix representation of $\phi$ given in (37) and observing that Lemma 9.3.1(ii) implies $\pi$ factors through the projection $F^{G} \rightarrow F_{1}^{G}$, one finds that

$$
\beta_{F^{\bullet}, G}\left(\operatorname{Tr}_{G}\left(b_{i}\right)\right)=\sum_{j \in|n|}\left(A_{i j}\left(\bmod I_{G, \Lambda}^{2}\right)\right) \otimes_{\Lambda} \pi\left(\operatorname{Tr}_{G}\left(b_{j}\right)\right) .
$$

This then implies the claimed result since $H^{1}\left(\varrho^{G}\right)\left(\pi\left(\operatorname{Tr}_{G}\left(b_{j}\right)\right)\right)=y_{j}$ by Lemma 9.3.1(ii)(a). 
We write $\overline{\operatorname{det}(A)}$ for the image of $\operatorname{det}(A)$ under the natural projection $I_{G, \Lambda}^{n} \rightarrow$ $\Lambda_{\alpha} \otimes_{\Lambda} I_{G, \Lambda}^{n} / I_{G, \Lambda}^{n+1}$. Then the description of Lemma 9.5.1 combines with the naturality with respect to the morphism $\varrho$ of the height pairing $\rho_{F} \bullet, G$ to imply that in $\Lambda_{\alpha} \otimes_{\Lambda} I_{G, \Lambda}^{n} / I_{G, \Lambda}^{n+1}$ one has

$$
\begin{aligned}
r_{\alpha, x ., y .} \overline{\operatorname{det}(A)} & =r_{\alpha, x ., y .} \operatorname{det}\left(\rho_{F \bullet, G}\left(\operatorname{Tr}_{G}\left(b_{i}\right),\left(H^{1}\left(\varrho^{G}\right)_{\mathbb{R}}^{-1}\left(y_{j}\right)\right)^{*}\right)_{1 \leq i, j \leq n}\right) \\
& =r_{\alpha, x ., y .} \operatorname{det}\left(\rho_{\Psi \bullet, G}\left(H^{0}\left(\varrho^{G}\right)\left(\operatorname{Tr}_{G}\left(b_{i}\right)\right), y_{j}^{*}\right)_{1 \leq i, j \leq n}\right) \\
& =r_{\alpha, x ., y .} \operatorname{det}(b) \operatorname{det}\left(\rho_{\Psi}, G\left(x_{i}, y_{j}^{*}\right)_{1 \leq i, j \leq n}\right) \\
& =\operatorname{det}(b) \operatorname{disc}_{\alpha}(\tau)
\end{aligned}
$$

where the third equality is a consequence of the definition of the matrix $b$ via the equalities (41).

Now $\left\{\operatorname{Tr}_{G}\left(b_{i}\right): 1 \leq i \leq n\right\}$ is a $\Lambda$-basis of $H^{0}\left(F^{\bullet}, G\right)$ (by Lemma 9.3.1(ii)(a)) whilst $\left\{x_{i}: 1 \leq i \leq n\right\}$ was chosen to be a $\Lambda$-basis of $\left(H^{0}\left(\Psi^{\bullet}\right)^{G}\right)_{\text {tf }}$ and so the definition of $b$ implies that $\operatorname{det}(b) \cdot \Lambda=\operatorname{Fit}_{\Lambda}\left(\operatorname{cok}\left(H^{0}\left(\varrho^{G}\right)^{\prime}\right)\right)$ where $H^{0}\left(\varrho^{G}\right)^{\prime}$ is the map $H^{0}\left(F^{\bullet} G\right) \rightarrow\left(H^{0}\left(\Psi^{\bullet}\right)^{G}\right)_{\mathrm{tf}}=\left(H^{0}\left(\Psi^{\bullet}\right) / T\right)^{G}$ induced by $H^{0}\left(\varrho^{G}\right)$. But, since $|Q / T|$ is coprime to $|G|$, the long exact sequence of cohomology of the exact sequence in Lemma 9.3.1(i) implies that $\left|\operatorname{cok}\left(H^{0}\left(\varrho^{G}\right)^{\prime}\right)\right|$ is coprime to $|G|$. Since $I_{G, \Lambda}^{n} / I_{G, \Lambda}^{n+1}$ is a finite group of order dividing a power of $|G|$ this means that multiplication by $\operatorname{det}(b)$ induces an automorphism of $\Lambda_{\alpha} \otimes_{\Lambda} I_{G, \Lambda}^{n} / I_{G, \Lambda}^{n+1}$. In particular, we may multiply the last displayed equality by $\operatorname{det}(b)^{-1}$ and in so doing deduce that $\operatorname{disc}_{\alpha}(\tau)$ is equal to the image of the element $r_{\alpha, x, y} \operatorname{det}(b)^{-1} \operatorname{det}(A)$ in $\Lambda_{\alpha} \otimes_{\Lambda} I_{G, \Lambda}^{n} / I_{G, \Lambda}^{n+1}$, as required.

This completes the proof of Theorem 9.2.2.

\section{Remark 9.5.2. Tate motives}

If $\tau=\tau_{0}$ as in Proposition 4.2.2, then $H_{\tau, \text { tor }}^{0}$ is equal to the torsion subgroup $\mu_{K}$ of $K^{\times}$and there are 'natural' choices of $\alpha$ in (Theorem 5.1.1(v) and) Theorem 9.2.2. Indeed, if $T$ is any finite set of places of $k$ disjoint from $S$ and such that the (finite index) subgroup of $\mathcal{O}_{K, S}^{\times}$comprising those elements congruent to 1 modulo all places in $T(K)$ is torsion-free, then the element $\alpha_{T}:=\prod_{v \in T}\left(1-\operatorname{Fr}_{v}^{-1} \mathrm{~N} v\right)$ belongs to $\mathrm{Fit}_{\mathbb{Z}[G]}\left(\mu_{K}\right)$, where $\mathrm{Fr}_{v}$ and $\mathrm{N} v$ are the frobenius automorphism of $v$ in $G$ and the absolute norm of $v$. In addition, $n_{\tau_{0}}=|S|-1$ and the explicit computation of Bockstein homomorphisms provided by $[17, \S 5.5]$ shows that if the conjectural equality $\chi\left(\tau_{0}\right)=0$ is valid, then the congruence of Theorem 9.2.2 
implies the 'refined class number formula' conjectured by Gross in [55, Conj. 4.1]. With further effort the hypothesis that the $\mathbb{Z}[G]$-module $\mu_{K}$ is perfect can be removed from this argument. For full details see [19].

Remark 9.5.3. Elliptic curves

If we assume that the conjectural equality $\chi(\tau)=0$ is valid for $\tau=\tau_{E, \mathbb{Z}\left[\frac{1}{2}\right]}$ as defined in Proposition 4.3.1 under condition (B), then the congruence of Theorem 9.2.2 is in precisely the same spirit as the 'Birch-Swinnerton-Dyer type conjecture' formulated by Mazur and Tate in [73, Conj. 4].

\section{Remark 9.5.4. Congruences for higher order derivatives}

If $e_{\tau}=0$, then the congruence of Theorem 9.2.2 is satisfied trivially (since (38) implies that both sides of the congruence vanish). However, in such cases it is still sometimes possible to derive from the conjectural vanishing of $\chi(\tau)$ a nontrivial congruence relation for the element $\mathcal{L}_{\tau}^{*} e_{\tau}^{\prime}$ with $e_{\tau}^{\prime}:=\sum_{\chi} e_{\chi}$ where $\chi$ runs over characters in $\operatorname{Irr}(G)$ with $\operatorname{dim}_{\mathbb{C}}\left(e_{\chi}\left(\mathbb{C} \otimes_{\Lambda} H_{\tau}^{0}\right)\right)=\min \left\{\operatorname{dim}_{\mathbb{C}}\left(e_{\psi}\left(\mathbb{C} \otimes_{\Lambda} H_{\tau}^{0}\right)\right)\right.$ : $\psi \in \operatorname{Irr}(G)\}$. The extent to which such congruences can be made explicit then depends upon the structure of $\left(H_{\tau}^{1}\right)_{\mathrm{tf}}$ as an abstract $G$-module. For example, if $\tau=\tau_{0}$, then $\left(H_{\tau}^{1}\right)_{\mathrm{tf}}=X_{K, S}$ has a very explicit structure and such an approach can be used to prove that $\operatorname{ETNC}\left(\mathbb{Q}(0)_{K}, \mathbb{Z}[G]\right)$ implies a natural generalisation and refinement of the conjectures formulated by Gross in [55], by Rubin in [86], by Popescu in [82] and by Tate in [95] and of the conjecture discussed by Hayward in [60]. For further details in this regard, and also for details of explicit connections between this approach and other 'refined abelian Stark conjectures', see [19]. (In the same setting, Macias Castillo has also recently shown that in many cases for which $e_{\tau}=0$ a variant of the approach of $[19]$ shows that $\operatorname{ETNC}\left(\mathbb{Q}(0)_{K}, \mathbb{Z}[G]\right)$ implies a generalisation of Stickelberger's Theorem in which the values of higherorder derivatives of $L$-functions are used to construct annihilators of natural class groups.) In the context of elliptic curves $E$ and extensions $K / \mathbb{Q}$ which together satisfy condition (B) of Proposition 4.3.1 the same approach shows that $\operatorname{ETNC}\left(h^{1}\left(E_{/ K}\right)(1), \mathbb{Z}^{\prime}[G]\right)$ with $\mathbb{Z}^{\prime}=\mathbb{Z}\left[\frac{1}{2}\right]$ implies explicit analogues of Theorem 9.2.2 in which $\mathcal{L}_{\tau_{E, \mathbb{Z}^{\prime}}}$ is replaced by an element which interpolates the values at $s=1$ of derivatives of Hasse-Weil $L$-functions. This interesting aspect of the theory will be further developed in a subsequent article. 


\section{A Hasse Principle for morphisms}

In this section we prove a natural local-to-global principle for morphisms in derived categories. This general result will be used in $\S 11$ and may well also be of some independent interest.

10.1. Statement of the result. We fix a $\mathbb{Z}$-order $\mathfrak{A}$ in a finite dimensional $\mathbb{Q}$ algebra $A$. For each prime $p$ we set $\mathfrak{A}_{p}:=\mathbb{Z}_{p} \otimes \mathfrak{A}$ and $A_{p}:=\mathbb{Q}_{p} \otimes_{\mathbb{Q}} A$. We also set $\mathfrak{D}:=D(\mathfrak{A})$ and $\mathfrak{D}_{p}:=D\left(\mathfrak{A}_{p}\right)$.

Theorem 10.1.1. (A Hasse Principle for morphisms) Let $C_{1}^{\bullet}$ and $C_{2}^{\bullet}$ be bounded complexes of finitely generated $\mathfrak{A}$-modules and, for each prime $p$, suppose given an element $\psi_{p}$ of $\operatorname{Hom}_{\mathfrak{D}_{p}}\left(\mathbb{Z}_{p} \otimes C_{1}^{\bullet}, \mathbb{Z}_{p} \otimes C_{2}^{\bullet}\right)$.

(i) There exists an element $\psi_{\mathbb{Z}}$ of $\operatorname{Hom}_{\mathfrak{D}}\left(C_{1}^{\bullet}, C_{2}^{\bullet}\right)$ with $\mathbb{Z}_{p} \otimes \psi_{\mathbb{Z}}=\psi_{p}$ for all primes $p$ if and only if for every integer $d$ there exists an element $\psi_{\mathbb{Q}}^{d}$ of $\operatorname{Hom}_{A}\left(H^{d}\left(\mathbb{Q} \otimes C_{1}^{\bullet}\right), H^{d}\left(\mathbb{Q} \otimes C_{2}^{\bullet}\right)\right)$ with $\mathbb{Q}_{p} \otimes_{\mathbb{Q}} \psi_{\mathbb{Q}}^{d}=\mathbb{Q}_{p} \otimes_{\mathbb{Z}_{p}} H^{d}\left(\psi_{p}\right)$ for all primes $p$.

(ii) If a morphism $\psi_{\mathbb{Z}}$ exists as in claim (i), then it is unique.

(iii) If a morphism $\psi_{\mathbb{Z}}$ exists as in claim (i), then it is an isomorphism in $\mathfrak{D}$ if and only if $\psi_{p}$ is an isomorphism in $\mathfrak{D}_{p}$ for all primes $p$.

The proof of this result will occupy the rest of this section.

10.2. A preliminary result. As a first step in proving Theorem 10.1.1 we consider a special case. More precisely, we assume to be given data of the following kind:-

- for each $i \in\{1,2\}$ we are given an exact triangle in $\mathfrak{D}$ of bounded complexes of finitely generated $\mathfrak{A}$-modules

$$
C_{i 1}^{\bullet} \rightarrow C_{i 2}^{\bullet} \rightarrow C_{i 3}^{\bullet} \rightarrow C_{i 1}^{\bullet}[1]
$$

- we are also given a commutative diagram of homomorphisms of the form 


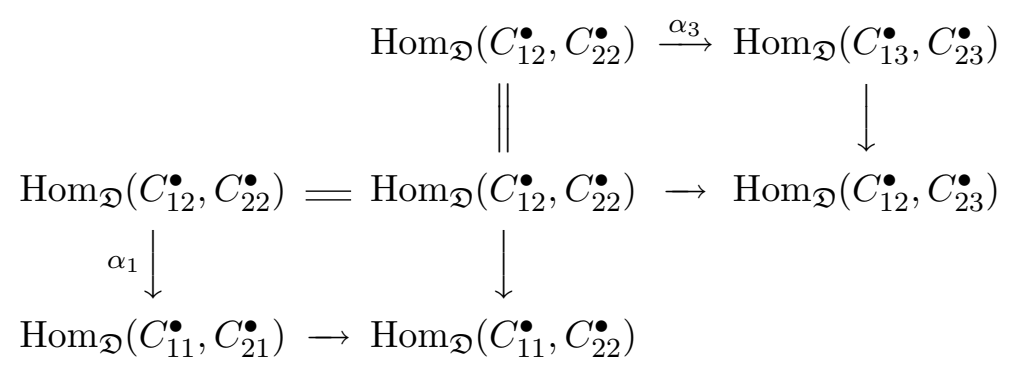

in which all unlabelled arrows represent the homomorphisms that are induced by the relevant exact triangles in (42).

\section{Example 10.2.1.}

(i) For each $i \in\{1,2\}$ we suppose that there is a finitely generated $\mathfrak{A}$-module $C_{i}$ such that $C_{i 2}^{\bullet}=C_{i}[0]$ and we take $C_{i 1}^{\bullet}=\left(C_{i}\right)_{\text {tor }}[0]$ and $C_{i 3}^{\bullet}=\left(C_{i}\right)_{\text {tf }}[0]$ respectively. We take $\alpha_{1}$ and $\alpha_{3}$ in (43) to be the obvious maps that are induced by the natural isomorphisms $\operatorname{Hom}_{\mathfrak{D}}\left(C_{12}^{\bullet}, C_{22}^{\bullet}\right) \cong \operatorname{Hom}_{\mathfrak{A}}\left(C_{1}, C_{2}\right), \operatorname{Hom}_{\mathfrak{D}}\left(C_{11}^{\bullet}, C_{21}^{\bullet}\right) \cong$ $\operatorname{Hom}_{\mathfrak{A}}\left(C_{1, \text { tor }}, C_{2, \text { tor }}\right)$ and $\operatorname{Hom}_{\mathfrak{D}}\left(C_{13}^{\bullet}, C_{23}^{\bullet}\right) \cong \operatorname{Hom}_{\mathfrak{A}}\left(C_{1, \mathrm{tf}}, C_{2, \mathrm{tf}}\right)$. We take (42) to be the triangle induced by the tautological exact sequences

$$
0 \rightarrow\left(C_{i}\right)_{\mathrm{tor}} \rightarrow C_{i} \rightarrow\left(C_{i}\right)_{\mathrm{tf}} \rightarrow 0 .
$$

(ii) For any complex $C^{\bullet}$ and integer $m$ we write $\sigma_{\leq m} C^{\bullet}$ and $\sigma_{>m} C^{\bullet}$ for the truncations of $C^{\bullet}$ in degrees less than or equal to $m$ and in degrees greater than $m$ respectively (so $H^{i}\left(\sigma_{\leq m} C^{\bullet}\right)=H^{i}\left(C^{\bullet}\right)$ and $H^{i}\left(\sigma_{>m} C^{\bullet}\right)=0$ if $i \leq m$ and $H^{i}\left(\sigma_{\leq m} C^{\bullet}\right)=0$ and $H^{i}\left(\sigma_{>m} C^{\bullet}\right)=H^{i}\left(C^{\bullet}\right)$ if $\left.i>m\right)$. We suppose given bounded complexes of finitely generated $\mathfrak{A}$-modules $C_{12}^{\bullet}$ and $C_{22}^{\bullet}$ and for both $i \in\{1,2\}$ we set $C_{i 1}^{\bullet}:=\sigma_{\leq k} C_{i 2}^{\bullet}$ and $C_{i 3}^{\bullet}:=\sigma_{>k} C_{i 2}^{\bullet}$ for some fixed integer $k$. Then we can take $\alpha_{1}$ and $\alpha_{3}$ in (43) to be induced by the functors $\sigma_{\leq k}$ and $\sigma_{>k}$ respectively and (42) to be the canonical exact triangle which relates $\sigma_{\leq k} C_{i 2}^{\bullet}, C_{i 2}^{\bullet}$ and $\sigma_{>k} C_{i 2}^{\bullet}$ (cf. [59, Chap. I, $\S 7,(2)]$ ).

With the above notation, for both $j=1$ and $j=3$ there is a natural commuting diagram

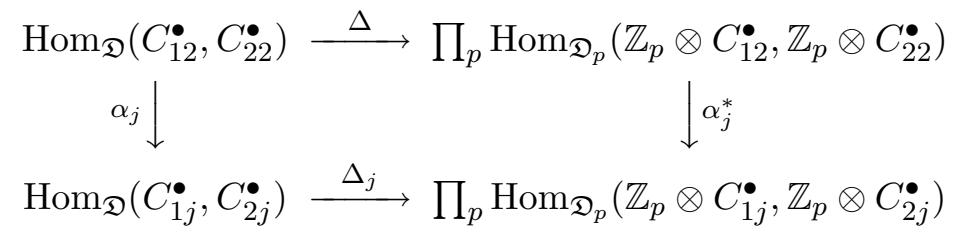


in which $\Delta$ and $\Delta_{j}$ are the obvious diagonal maps, and $\alpha_{j}^{*}=\prod_{p}\left(\mathbb{Z}_{p} \otimes \alpha_{j}\right)$.

Proposition 10.2.2. We assume to be given data as in (42) and (43). We also assume that there exists an integer $k$ such that the following three conditions are satisfied:-

(a) For both $i \in\{1,2\}$ one has $H^{a}\left(C_{i 1}^{\bullet}\right)=0$ in all degrees $a>k$ and $H^{b}\left(C_{i 3}^{\bullet}\right)=0$ in all degrees $b<k$;

(b) $\operatorname{Hom}_{\mathfrak{A}}\left(H^{k}\left(C_{13}^{\bullet}\right), H^{k}\left(C_{21}^{\bullet}\right)\right)$ is finite;

(c) $\operatorname{Hom}_{\mathfrak{A}}\left(H^{k}\left(C_{11}^{\bullet}\right), H^{k}\left(C_{23}^{\bullet}\right)\right)=0$.

Then for any element $\psi$ of $\prod_{p} \operatorname{Hom}_{\mathfrak{D}_{p}}\left(\mathbb{Z}_{p} \otimes C_{12}^{\bullet}, \mathbb{Z}_{p} \otimes C_{22}^{\bullet}\right)$ one has $\psi \in \operatorname{im}(\Delta)$ if and only if $\alpha_{j}^{*}(\psi) \in \operatorname{im}\left(\Delta_{j}\right)$ for both $j \in\{1,3\}$, where $\alpha_{j}^{*}$ and $\Delta_{j}$ are as in diagram (44).

Proof. For each pair of complexes of $\mathfrak{A}$-modules $X^{\bullet}$ and $Y^{\bullet}$ we set $\left\langle X^{\bullet}, Y^{\bullet}\right\rangle:=$ $\operatorname{Hom}_{\mathfrak{D}}\left(X^{\bullet}, Y^{\bullet}\right)$. Then from the given exact triangles $(42)$ we obtain a commutative diagram of abelian groups with exact rows and columns

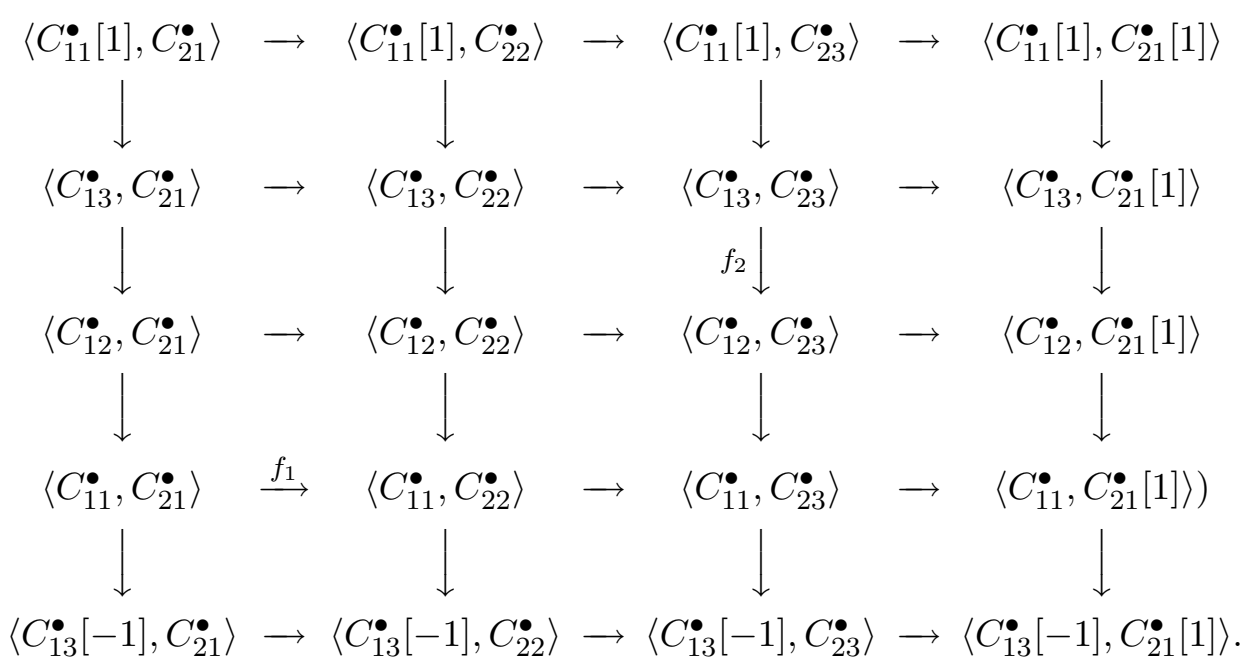

To analyse this diagram we use the following observations.

\section{Lemma 10.2.3.}

(i) For any integers $r$ and $s$ with $r \geq s$ one has $\left\langle C_{11}^{\bullet}[r], C_{23}^{\bullet}[s]\right\rangle=0$. 
(ii) The group $\left\langle C_{13}^{\bullet}, C_{21}^{\bullet}\right\rangle$ is finite.

Proof. There are natural isomorphisms

$$
\left\langle C_{11}^{\bullet}[r], C_{23}^{\bullet}[s]\right\rangle \cong\left\langle C_{11}^{\bullet}, C_{23}^{\bullet}[s-r]\right\rangle \cong H^{0}\left(R \operatorname{Hom}_{\mathfrak{A}}\left(C_{11}^{\bullet}, C_{23}^{\bullet}[s-r]\right)\right),
$$

and the latter group can be computed by using the spectral sequence

$$
E_{2}^{p, q}=\prod_{t \in \mathbb{Z}} \operatorname{Ext}_{\mathfrak{A}}^{p}\left(H^{t}\left(C_{11}^{\bullet}\right), H^{q+t+s-r}\left(C_{23}^{\bullet}\right)\right) \Rightarrow H^{p+q}\left(R \operatorname{Hom}_{\mathfrak{A}}\left(C_{11}^{\bullet}, C_{23}^{\bullet}[s-r]\right)\right)
$$

from [96, III, 4.6.10]. But condition (a) implies that $H^{t}\left(C_{11}^{\bullet}\right)=0$ for $t>k$ and that $H^{q+t+s-r}\left(C_{23}^{\bullet}\right)=0$ for $t<k+(r-s)-q$. Hence, since both $r \geq s$ and $\operatorname{Ext}_{\mathfrak{A}}^{p}(-,-)=0$ for $p<0$, it follows that

$$
H^{0}\left(R \operatorname{Hom}_{\mathfrak{A}}\left(C_{11}^{\bullet}, C_{23}^{\bullet}[s-r]\right)= \begin{cases}0, & \text { if } r>s \\ \operatorname{Hom}_{\mathfrak{A}}\left(H^{k}\left(C_{11}^{\bullet}\right), H^{k}\left(C_{23}^{\bullet}\right)\right), & \text { if } r=s .\end{cases}\right.
$$

Claim (i) is therefore a consequence of condition (c) in Proposition 10.2.2.

Claim (ii) is proved by making a similar calculation of $\left\langle C_{13}^{\bullet}, C_{21}^{\bullet}\right\rangle$ and then using both condition (b) of Proposition 10.2.2 and the following fact: for any finitely generated $\mathfrak{A}$-modules $M$ and $N$ and any strictly positive integer $p$ the group $\operatorname{Ext}_{\mathfrak{A}}^{p}(M, N)$ is finite.

Lemma 10.2.3(i) implies that the maps labelled $f_{1}$ and $f_{2}$ in the above commutative diagram are bijective. When combined with the given commutative diagram (43) this fact gives rise to an exact sequence of the form

$$
\left\langle C_{13}^{\bullet}, C_{21}^{\bullet}\right\rangle \rightarrow\left\langle C_{12}^{\bullet}, C_{22}^{\bullet}\right\rangle \stackrel{\left(\alpha_{1}, \alpha_{3}\right)}{\longrightarrow}\left\langle C_{11}^{\bullet}, C_{21}^{\bullet}\right\rangle \oplus\left\langle C_{13}^{\bullet}, C_{23}^{\bullet}\right\rangle \rightarrow Q
$$

with $Q:=\operatorname{cok}\left(\left(\alpha_{1}, \alpha_{3}\right)\right)$.

For each prime $p$ and each set of integers $i, j, r, s$ we define $\langle i j ; r s\rangle_{p}:=\operatorname{Hom}_{\mathfrak{D}_{p}}\left(\mathbb{Z}_{p} \otimes\right.$ $\left.C_{i j}^{\bullet}, \mathbb{Z}_{p} \otimes C_{r s}^{\bullet}\right)$. Then by repeating the above argument one obtains for each prime $p$ a precise analogue of the exact sequence (46) with each complex $C_{i j}^{\bullet}$ replaced by $\mathbb{Z}_{p} \otimes C_{i j}^{\bullet}$, and hence a natural commutative diagram

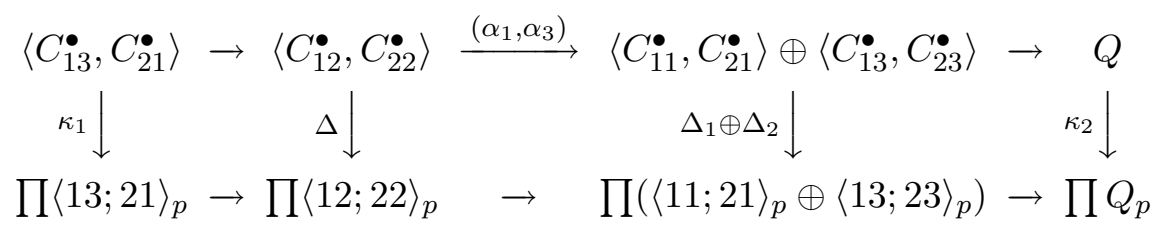


in which the products run over all primes $p$ and both rows are exact. To analyse this diagram we use the following result.

Lemma 10.2.4. If $X$ and $Y$ are bounded complexes of finitely generated $\mathfrak{A}$ modules, then for each $p$ the canonical homomorphism $\mathbb{Z}_{p} \otimes \operatorname{Hom}_{\mathfrak{D}}(X, Y) \rightarrow$ $\operatorname{Hom}_{\mathfrak{D}_{p}}\left(\mathbb{Z}_{p} \otimes X, \mathbb{Z}_{p} \otimes Y\right)$ is bijective. In particular therefore, the canonical map from $\operatorname{Hom}_{\mathfrak{D}}(X, Y)$ to $\prod_{p} \operatorname{Hom}_{\mathfrak{D}_{p}}\left(\mathbb{Z}_{p} \otimes X, \mathbb{Z}_{p} \otimes Y_{p}\right)$, where $p$ runs over all primes, is injective, resp. bijective if $\operatorname{Hom}_{\mathfrak{D}}(X, Y)$ is finite.

Proof. See [22, proof of Lem. 17].

In the context of diagram (47), Lemma 10.2.4 implies that $Q_{p}$ is canonically isomorphic to $\mathbb{Z}_{p} \otimes Q$ for each $p$ and hence that the map $\kappa_{2}$ is injective. In addition, Lemma 10.2.4 also combines with Lemma 10.2.3(ii) to imply that the map $\kappa_{1}$ in (47) is bijective. Using these facts the result of Proposition 10.2.2 follows by an easy diagram chase using (47).

10.3. The proof of Theorem 10.1.1. To prove Theorem 10.1.1(i) we set $\ell\left(C_{i}^{\bullet}\right)$ $:=\left\{d \in \mathbb{Z}: H^{d}\left(C_{i}^{\bullet}\right) \neq 0\right\}$ and argue by induction on $\left|\ell\left(C_{1}^{\bullet}\right)\right|+\left|\ell\left(C_{2}^{\bullet}\right)\right|$. If firstly $\left|\ell\left(C_{1}^{\bullet}\right)\right|+\left|\ell\left(C_{2}^{\bullet}\right)\right|=0$, then the complexes $C_{1}^{\bullet}, C_{2}^{\bullet}, \mathbb{Z}_{p} \otimes C_{1}^{\bullet}$ and $\mathbb{Z}_{p} \otimes C_{2}^{\bullet}$ are all acyclic and so Theorem 10.1.1(i) is obvious. To deal with the inductive step we observe first that unless $\ell\left(C_{1}^{\bullet}\right)=\ell\left(C_{2}^{\bullet}\right)$ and $\left|\ell\left(C_{1}^{\bullet}\right)\right|=1$, then there exists an integer $k$ such that $\left|\ell\left(\sigma_{\leq k} C_{1}^{\bullet}\right)\right|+\left|\ell\left(\sigma_{\leq k} C_{2}^{\bullet}\right)\right|$ and $\left|\ell\left(\sigma_{>k} C_{1}^{\bullet}\right)\right|+\left|\ell\left(\sigma_{>k} C_{2}^{\bullet}\right)\right|$ are both strictly less than $\left|\ell\left(C_{1}^{\bullet}\right)\right|+\left|\ell\left(C_{2}^{\bullet}\right)\right|$. Hence, if either $\ell\left(C_{1}^{\bullet}\right) \neq \ell\left(C_{2}^{\bullet}\right)$ or $\left|\ell\left(C_{1}^{\bullet}\right)\right| \neq 1$, then Theorem 10.1.1(i) can be deduced by fixing such an integer $k$, applying Proposition 10.2.2 in the context of Example 10.2.1(ii) (it is easy to check that the conditions (a), (b) and (c) of Proposition 10.2.2 are satisfied in this setting) and then using the inductive hypothesis with $C_{1}^{\bullet}$ and $C_{2}^{\bullet}$ replaced by $\sigma_{\leq k} C_{1}^{\bullet}$ and $\sigma_{\leq k} C_{2}^{\bullet}$, resp. by $\sigma_{>k} C_{1}^{\bullet}$ and $\sigma_{>k} C_{2}^{\bullet}$. On the other hand, if $\ell\left(C_{1}^{\bullet}\right)=\ell\left(C_{2}^{\bullet}\right)=\{d\}$ for some integer $d$, then there exist finitely generated $\mathfrak{A}$-modules $X$ and $Y$ such that $C_{1}^{\bullet}$ and $C_{2}^{\bullet}$ are isomorphic in $\mathfrak{D}$ to $X[-d]$ and $Y[-d]$ respectively. Hence, in this case $\operatorname{Hom}_{\mathfrak{D}}\left(C_{1}^{\bullet}, C_{2}^{\bullet}\right)$ and $\operatorname{Hom}_{\mathfrak{D}_{p}}\left(\mathbb{Z}_{p} \otimes C_{1}^{\bullet}, \mathbb{Z}_{p} \otimes C_{2}^{\bullet}\right)$ are naturally isomorphic to $\operatorname{Hom}_{\mathfrak{A}}(X, Y)$ and $\operatorname{Hom}_{\mathfrak{A}_{p}}\left(\mathbb{Z}_{p} \otimes X, \mathbb{Z}_{p} \otimes Y\right)$ respectively, and so the assertion of Theorem 10.1.1(i) is equivalent to the following result.

Lemma 10.3.1. Let $X$ and $Y$ be finitely generated $\mathfrak{A}$-modules and for each prime $p$ let $\psi_{p}$ be an element of $\operatorname{Hom}_{\mathfrak{A}_{p}}\left(\mathbb{Z}_{p} \otimes X, \mathbb{Z}_{p} \otimes Y\right)$. Then there exists an element 
$\psi$ of $\operatorname{Hom}_{\mathfrak{A}}(X, Y)$ with $\mathbb{Z}_{p} \otimes \psi=\psi_{p}$ for all primes $p$ if and only if there exists an element $\psi_{\mathbb{Q}}$ of $\operatorname{Hom}_{A}(\mathbb{Q} \otimes X, \mathbb{Q} \otimes Y)$ with $\mathbb{Q}_{p} \otimes_{\mathbb{Q}} \psi_{\mathbb{Q}}=\mathbb{Q}_{p} \otimes_{\mathbb{Z}_{p}} \psi_{p}$ for all primes $p$.

Proof. This follows by applying Proposition 10.2.2 (with $k=0$ ) in the context of Example 10.2.1(i) (it is easy to check that the conditions (a), (b) and (c) of Proposition 10.2.2 are satisfied in this setting). Indeed, in this case Lemma 10.2.4 implies that the map $\Delta_{1}$ in diagram (44) is bijective and so Proposition 10.2.2 implies that $\prod_{p} \psi_{p}$ belongs to $\operatorname{im}(\Delta)$ if and only if there exists an element $\psi_{\mathrm{tf}}$ of $\operatorname{Hom}_{\mathfrak{A}}\left(X_{\mathrm{tf}}, Y_{\mathrm{tf}}\right)$ with $\mathbb{Z}_{p} \otimes \psi_{\mathrm{tf}}=\left(\mathbb{Z}_{p} \otimes \alpha_{3}\right)\left(\psi_{p}\right)$ for all primes $p$. But the existence of such a homomorphism $\psi_{\mathrm{tf}}$ is easily seen to be equivalent to the existence of a homomorphism $\psi_{\mathbb{Q}}$ as in the statement of the lemma.

This completes our proof of Theorem 10.1.1(i). Further, if the morphism $\psi_{\mathbb{Z}}$ exists as in claim (i), then Lemma 10.2.4 implies that it is unique (and this proves Theorem 10.1.1(ii)). In addition, it is clear that $\psi_{\mathbb{Z}}$ is an isomorphism in $\mathfrak{D}$ if and only if the homomorphism $H^{d}\left(\psi_{\mathbb{Z}}\right)$ is bijective in all degrees $d$. Claim (iii) of Theorem 10.1.1 thus follows directly from the fact that $\mathbb{Z}_{p} \otimes \operatorname{ker}\left(\psi_{\mathbb{Z}}\right)=\operatorname{ker}\left(\psi_{p}\right)$ and $\mathbb{Z}_{p} \otimes \operatorname{cok}\left(\psi_{\mathbb{Z}}\right)=\operatorname{cok}\left(\psi_{p}\right)$ for all primes $p$. This completes our proof of Theorem 10.1.1.

\section{TAte motives of STRICTly POSITIVe Weight}

In this section we prove Proposition 4.2.6 and the relevant claims in both Remarks 6.2.4 and 7.1.3.

11.1. The proof of Proposition 4.2.6. At the outset we fix a finite set of places $S$ of $k$ as in $\S 4.2$ and a strictly negative integer $r$ and define a $G$-module by setting

$$
H_{r}^{+}:=\left(\bigoplus_{\operatorname{Hom}(K, \mathbb{C})}(2 \pi i)^{-r} \mathbb{Z}\right)^{\operatorname{Gal}(\mathbb{C} / \mathbb{R})}
$$

where $\operatorname{Gal}(\mathbb{C} / \mathbb{R})$ acts diagonally on the direct sum. For each prime $p$ we also set

$$
C_{p, r}^{\bullet}:=R \operatorname{Hom}_{\mathbb{Z}_{p}}\left(R \Gamma_{c}\left(\mathcal{O}_{K, S}\left[\frac{1}{p}\right], \mathbb{Z}_{p}(r)\right), \mathbb{Z}_{p}[-2]\right)
$$

where $R \Gamma_{c}\left(\mathcal{O}_{K, S}\left[\frac{1}{p}\right], \mathbb{Z}_{p}(r)\right)$ is the complex of $\mathbb{Z}_{p}[G]$-modules given by the cohomology with compact support defined in [23, p. 522]. We assume throughout that the Chern class maps $\operatorname{ch}_{p, r}^{1}$ and $\operatorname{ch}_{p, r}^{2}$ in (10) are bijective for all odd primes $p$. 
Lemma 11.1.1. The complex $C_{p, r}^{\bullet}$ belongs to $D^{\text {perf }}\left(\mathbb{Z}_{p}[G]\right)$. It is also acyclic outside degrees 0 and 1 and there are canonical isomorphisms of $\mathbb{Z}_{p}[G]$-modules of the form:-

(i) $H^{0}\left(C_{p, r}^{\bullet}\right) \cong \mathbb{Z}_{p} \otimes K_{1-2 r}\left(\mathcal{O}_{K}\right)$ if either $p$ is odd, or $p=2$ and $K$ is totally imaginary, or $p=2$ and $r \equiv 0,1(\bmod 4)$;

(ii) $H^{1}\left(C_{p, r}^{\bullet}\right)_{\text {tor }} \cong \mathbb{Z}_{p} \otimes K_{-2 r}\left(\mathcal{O}_{K, S}\right)$ if either $p$ is odd, or $p=2$ and $K$ is totally imaginary, or $p=2$ and $r \equiv 0,3(\bmod 4)$;

(iii) $H^{1}\left(C_{p, r}^{\bullet}\right)_{\mathrm{tf}} \cong \mathbb{Z}_{p} \otimes H_{r}^{+}$if either $p$ is odd, or $p=2$ and $K$ is totally imaginary. In general one has $\mathbb{Q}_{2} \otimes_{\mathbb{Z}_{2}} H^{1}\left(C_{2, r}^{\bullet}\right)_{\mathrm{tf}} \cong \mathbb{Q}_{2} \otimes H_{r}^{+}$.

Proof. For each prime $p$ set $D_{p, r}^{\bullet}:=R \Gamma_{c}\left(\mathcal{O}_{K, S}\left[\frac{1}{p}\right], \mathbb{Z}_{p}(r)\right)$. Then it is well known that $D_{p, r}^{\bullet}$, and hence also its (shifted) linear dual $C_{p, r}^{\bullet}$, belongs to $D^{\text {perf }}\left(\mathbb{Z}_{p}[G]\right)$ and that $D_{p, r}^{\bullet}$ is acyclic outside degrees 1,2 and 3 (indeed, for more general results see [44, Th. 5.1]). One also knows that $H^{3}\left(D_{p, r}^{\bullet}\right)$ is finite and it is straightforward to show that $H^{1}\left(D_{p, r}^{\bullet}\right)$ is $\mathbb{Z}_{p}$-torsion-free and, taken in conjunction with the universal coefficient spectral sequence, these facts imply that $C_{p, r}^{\bullet}$ is acyclic outside degrees 0 and 1 . Further, global duality induces (via, for example, the long exact cohomology sequence of the central column of the diagram in [22, Prop. 4.1]) canonical isomorphims $H^{0}\left(C_{p, r}^{\bullet}\right) \cong H_{\text {ét }}^{1}\left(\operatorname{Spec}\left(\mathcal{O}_{K, S}\left[\frac{1}{p}\right]\right), \mathbb{Z}_{p}(r)\right)$ and $H^{1}\left(C_{p, r}^{\bullet}\right)_{\text {tor }} \cong H_{\text {ét }}^{2}\left(\operatorname{Spec}\left(\mathcal{O}_{K, S}\left[\frac{1}{p}\right]\right), \mathbb{Z}_{p}(r)\right)$ and also $\Lambda \otimes_{\mathbb{Z}_{p}} H^{1}\left(C_{p, r}^{\bullet}\right)_{\mathrm{tf}} \cong \Lambda \otimes_{\mathbb{Z}_{p}}$ $\bigoplus_{w} H^{0}\left(K_{w}, \mathbb{Z}_{p}(-r)\right)$ where in the last sum $w$ runs over all archimedean places of $K$ and $\Lambda$ is equal to $\mathbb{Z}_{p}$ if either $p$ is odd or $K$ totally imaginary and is otherwise equal to $\mathbb{Q}_{2}$.

Given the above description of $H^{0}\left(C_{p, r}^{\bullet}\right)$ the isomorphism in claim (i) results from the fact that the natural localisation map $K_{1-2 r}\left(\mathcal{O}_{K}\right) \rightarrow K_{1-2 r}\left(\mathcal{O}_{K, S}\right)$ is bijective (see, for example, [33, Prop. 5.7]), our assumption that $\operatorname{ch}_{p, r}^{1}$ is bijective if $p$ is odd and the fact that $\operatorname{ch}_{2, r}^{1}$ is known to be bijective if either $K$ is totally imaginary or $r \equiv 0,1(\bmod 4)$ and that in all cases $\mathbb{Q}_{2} \otimes_{\mathbb{Z}_{2}} \operatorname{ch}_{2, r}^{1}$ is known to be bijective [63, Th. 1]. In a similar way, the isomorphism in claim (ii) results from our assumption that $\operatorname{ch}_{p, r}^{2}$ is bijective if $p$ is odd and the fact that $\operatorname{ch}_{2, r}^{2}$ is known to be bijective if either $K$ is totally imaginary or $r \equiv 0,3(\bmod 4)[63$, Th. 1]. Lastly, the isomorphisms in claim (iii) are obtained by combining the above description of $\Lambda \otimes_{\mathbb{Z}_{p}} H^{1}\left(C_{p, r}^{\bullet}\right)_{\mathrm{tf}}$ with the canonical $p$-adic comparison isomorphism $\bigoplus_{w} H^{0}\left(K_{w}, \mathbb{Z}_{p}(-r)\right) \cong \mathbb{Z}_{p} \otimes H_{r}^{+}$(which depends on the choice of a topological generator of $\left.\mathbb{Z}_{p}(-r)\right)$. 
The descriptions of Lemma 11.1.1(i) and (iii) combine with [37, Prop. 4.21] to prove the existence of a (unique) full $\mathbb{Z}[G]$-sublattice $N_{r, 0}$ of $\mathbb{Q} \otimes K_{1-2 r}\left(\mathcal{O}_{K}\right)$ and $N_{r, 1}$ of $\mathbb{Q} \otimes H_{r}^{+}$with the property that for both $a=0,1$ the maps in Lemma 11.1.1(i) and (iii) induce isomorphisms

$$
\mu_{p, a}: \mathbb{Z}_{p} \otimes N_{r, a} \cong H^{a}\left(C_{p, r}^{\bullet}\right)_{\mathrm{tf}}
$$

for all primes $p$. From the descriptions of Lemma 11.1.1(i) and (ii) we also know that, for both $a=0,1$, the module $\bigoplus_{p} H^{a}\left(C_{p, r}^{\bullet}\right)$ tor is finite, where in the sum $p$ runs over all primes.

Now for any finitely generated $G$-modules $M$ and $N$ and any strictly positive integer $i$ there is a natural isomorphism

$$
\operatorname{Ext}_{\mathbb{Z}[G]}^{i}(N, M) \cong \bigoplus_{p \| G \mid} \operatorname{Ext}_{\mathbb{Z}_{p}[G]}^{i}\left(\mathbb{Z}_{p} \otimes N, \mathbb{Z}_{p} \otimes M\right)
$$

In particular, for both $a=0,1$ this observation (with $i=1, N=N_{r, a}$ and $\left.M=\bigoplus_{p} H^{a}\left(C_{p, r}^{\bullet}\right)_{\text {tor }}\right)$ proves the existence of a finitely generated $G$-module $N_{r, a}^{\prime}$ such that for every prime $p$ there exists an isomorphism of $\mathbb{Z}_{p}[G]$-modules

$$
\mu_{p, a}^{\prime}: \mathbb{Z}_{p} \otimes N_{r, a}^{\prime} \cong H^{a}\left(C_{p, r}^{\bullet}\right)
$$

which induces on $\mathbb{Z}_{p} \otimes\left(N_{r, a}^{\prime}\right)_{\mathrm{tf}}$ the isomorphism $\mu_{p, a}$ fixed above.

By now applying (48) with $i=2, N=N_{r, 1}^{\prime}$ and $M=N_{r, 0}^{\prime}$, the isomorphisms $\mu_{p, 0}^{\prime}$ and $\mu_{p, 1}^{\prime}$ combine to give an isomorphism

$$
\operatorname{Ext}_{\mathbb{Z}[G]}^{2}\left(N_{r, 1}^{\prime}, N_{r, 0}^{\prime}\right) \cong \bigoplus_{p \| G \mid} \operatorname{Ext}_{\mathbb{Z}_{p}[G]}^{2}\left(H^{1}\left(C_{p, r}^{\bullet}\right), H^{0}\left(C_{p, r}^{\bullet}\right)\right) .
$$

Hence we may define an a.t.e. of $\mathbb{Z}[G]$-modules $\tau_{r}$ by means of the following conditions:-

- $H_{\tau_{r}}^{a}=N_{r, a}^{\prime}$ for both $a=0,1$;

- $\epsilon_{\tau_{r}}$ is the element of $\operatorname{Ext}_{\mathbb{Z}[G]}^{2}\left(H_{\tau_{r}}^{1}, H_{\tau_{r}}^{0}\right)$ that corresponds under (50) to $\bigoplus_{p|| G \mid} C_{p, r}^{\bullet}$ (note that $\epsilon_{\tau_{r}}$ is perfect since $C_{p, r}^{\bullet}$ belongs to $D^{\text {perf }}\left(\mathbb{Z}_{p}[G]\right)$ for each prime divisor $p$ of $|G|)$;

- $\lambda_{\tau_{r}}$ is induced by -1 times the Beilinson regulator map;

- $\mathcal{L}_{\tau_{r}}^{*}=L_{K / k, S}^{*}(r)^{\#}$.

With this construction of $\tau_{r}$ the descriptions of $H_{\tau_{r}}^{0}$ and $H_{\tau_{r}}^{1}$ given in Proposition 4.2 .6 are an easy consequence of the isomorphisms $\mu_{p, a}^{\prime}$ for $a=0,1$ and 
the descriptions of $H^{a}\left(C_{p, r}^{\bullet}\right)$ in Lemma 11.1.1. The proof of Proposition 4.2.6 is therefore now completed by the following result.

Lemma 11.1.2. $\psi^{*}\left(T \Omega\left(\mathbb{Q}(r)_{K}, \mathbb{Z}[G]\right)\right)=\chi\left(\tau_{r}\right)$.

Proof. By [13, Lem. 2.1], it suffices to prove that for each prime $p$ and embedding $j: \mathbb{R} \rightarrow \mathbb{C}_{p}$ one has $\iota_{j}\left(T \Omega\left(\mathbb{Q}(r)_{K}, \mathbb{Z}[G]\right)\right)=\iota_{j}\left(\psi^{*}\left(\chi\left(\tau_{r}\right)\right)\right)$ with $\iota_{j}$ the homomorphism $K_{0}(\mathbb{Z}[G], \mathbb{R}[G]) \rightarrow K_{0}\left(\mathbb{Z}_{p}[G], \mathbb{C}_{p}[G]\right)$ induced by $j$.

But (5) implies that $\iota_{j}\left(\psi^{*}\left(\chi\left(\tau_{r}\right)\right)\right)=\left[D_{p, r}^{\bullet}, \lambda_{j}\right]+\iota_{j}\left(\delta\left(L_{K / k, S}^{*}(r)\right)\right)$ with $D_{p, r}^{\bullet}:=$ $R \Gamma_{c}\left(\mathcal{O}_{K, S}\left[\frac{1}{p}\right], \mathbb{Z}_{p}(r)\right)$ and $\lambda_{j}:\left[\mathbb{C}_{p} \otimes_{\mathbb{Z}_{p}} H^{1}\left(D_{p, r}^{\bullet}\right)\right]^{-1}\left[\mathbb{C}_{p} \otimes_{\mathbb{Z}_{p}} H^{2}\left(D_{p, r}^{\bullet}\right)\right] \rightarrow \mathbf{1}_{\mathbb{C}_{p}[G]}$ the morphism in $V\left(\mathbb{C}_{p}[G]\right)$ that is induced by (the $\mathbb{C}_{p}$-linear duals of) $\mathbb{C}_{p} \otimes_{\mathbb{R}, j} \lambda_{\tau_{r}}$ and the image under $\mathbb{C}_{p} \otimes_{\mathbb{Z}_{p}}$ - of the isomorphisms in Lemma 11.1.1. The required equality thus follows because $\left[D_{p, r}^{\bullet}, \lambda_{j}\right]$ is equal to the image under $\iota_{j}$ of the element

$$
\begin{aligned}
T \Omega\left(\mathbb{Q}(r)_{K}, \mathbb{Z}[G]\right)-\delta\left(L_{K / k, S}^{*}(r)\right) \\
=\left(T \Omega\left(\mathbb{Q}(r)_{K}, \mathbb{Z}[G]\right)-\delta\left(L_{K / k, S_{\infty}}^{*}(r)\right)\right)-\delta\left(L_{K / k, S}^{*}(r) / L_{K / k, S_{\infty}}^{*}(r)\right) .
\end{aligned}
$$

Indeed, if $G$ is abelian, then the latter claim can be proved by comparing the definition of $\left[D_{p, r}^{\bullet}, \lambda_{j}\right]$ with the definition of $T \Omega\left(\mathbb{Q}(r)_{K}, \mathbb{Z}[G]\right)-\delta\left(L_{K / k, S_{\infty}}^{*}(r)\right)$ via $[25,(9)]$ and $[23$, Conj. 4(iii)] and then using $[22,(11),(12)]$ and the fact that (in the notation of loc. cit.) $1-\mathrm{N} v^{-r} f_{v}$ belongs to $\mathbb{Z}_{p}[G]^{\times}$for each place $v$ of $k$ above $p$. The formalism of virtual objects then allows a direct extension of this argument to the general non-abelian case.

11.2. Justifying Remark 7.1.3. In this subsection we prove the equality $\chi\left(\epsilon_{\tau_{r}}\right)=$ $\Omega_{-r}(K / k)$ that is used in Remark 7.1.3. To do this we apply Theorem 10.1.1 in the setting of $[22, \S 4]$. We therefore first recall that

$$
\Omega_{-r}(K / k)=\chi(K(1-r))-\left(\mathbb{Z} \Sigma_{\infty}\right) \in K_{0}(\mathbb{Z}[G]),
$$

where $K(1-r)$ is the perfect complex of $\mathbb{Z}[G]$-module which occurs in $[22,(106)]$ and $\Sigma_{\infty}$ is the $G$-set $\operatorname{Hom}(K, \mathbb{C})$. We also recall from loc. cit. that there exists a perfect complex of $\mathbb{Z}[G]$-modules $\Delta:=\Delta(1-r)$ with all of the following properties:-

(a) For each prime $p$ the left hand column of [22, Prop. 4.1] combines with the isomorphisms $\tau_{p}$ and $\sigma_{p}$ of $[22,(106)$, resp. Prop. 4.2] to give an exact 
triangle in $D\left(\mathbb{Z}_{p}[G]\right)$ of the form

$$
\mathbb{Z}_{p} \otimes \Delta[1] \stackrel{\psi_{p}}{\longrightarrow} \mathbb{Z}_{p} \otimes K(1-r) \rightarrow C_{p, r}^{\bullet} \rightarrow \mathbb{Z}_{p} \otimes \Delta[2] ;
$$

(b) [22, Prop. 4.2] implies that in each degree $i$ there exists a homomorphism of $\mathbb{Q}[G]$-modules $\psi_{\mathbb{Q}}^{i}: \mathbb{Q} \otimes H^{i+1}(\Delta) \rightarrow \mathbb{Q} \otimes H^{i}(K(1-r))$ which makes the following diagram commute

$$
\begin{aligned}
& \mathbb{Q}_{p} \otimes H^{i+1}(\Delta) \quad \stackrel{\mathbb{Q}_{p} \otimes_{\mathbb{Q}} \psi_{\mathbb{Q}}^{i}}{\longrightarrow} \quad \mathbb{Q}_{p} \otimes H^{i}(K(1-r)) \\
& \cong \downarrow \\
& H^{i+1}\left(\mathbb{Q}_{p} \otimes \Delta\right) \stackrel{\mathbb{Q}_{p} \otimes_{\mathbb{Z}_{p}} H^{i}\left(\psi_{p}\right)}{\longrightarrow} H^{i}\left(\mathbb{Q}_{p} \otimes K(1-r)\right),
\end{aligned}
$$

where the vertical arrows are the canonical isomorphisms;

(c) $\Delta$ is isomorphic in $D(\mathbb{Z}[G])$ to a complex $\mathbb{Z} \Sigma_{\infty} \rightarrow \mathbb{Z} \Sigma_{\infty} \rightarrow \mathbb{Z} \Sigma_{\infty}$, where the first term is placed in degree 1 (cf. [22, Lem. 18]).

Property (b) implies that the criterion of Theorem 10.1.1(i) is satisfied with $\mathfrak{A}=\mathbb{Z}[G], C_{1}^{\bullet}=\Delta[1], C_{2}^{\bullet}=K(1-r)$ and $\psi_{p}$ as in property (a) and hence there exists a morphism $\psi_{\mathbb{Z}}: \Delta[1] \rightarrow K(1-r)$ in $D(\mathbb{Z}[G])$ with $\mathbb{Z}_{p} \otimes \psi_{\mathbb{Z}}=\psi_{p}$ for all primes $p$.

Using the terminology of $\S 3$, we now fix a representative $\left(\Psi^{\bullet}, \iota_{0}, \iota_{1}\right)$ for the extension class $\epsilon_{\tau_{r}}$ defined just prior to Lemma 11.1.2. If $X$ is any complex of $\mathbb{Z}[G]$-modules which lies in an exact triangle in $D(\mathbb{Z}[G])$ of the form

$$
\Delta[1] \stackrel{\psi_{\mathbb{Z}}}{\longrightarrow} K(1-r) \rightarrow X \rightarrow \Delta[2],
$$

then for each prime $p$ there exists an isomorphism $\lambda_{p}$ in $D\left(\mathbb{Z}_{p}[G]\right)$ which makes the following diagram commute

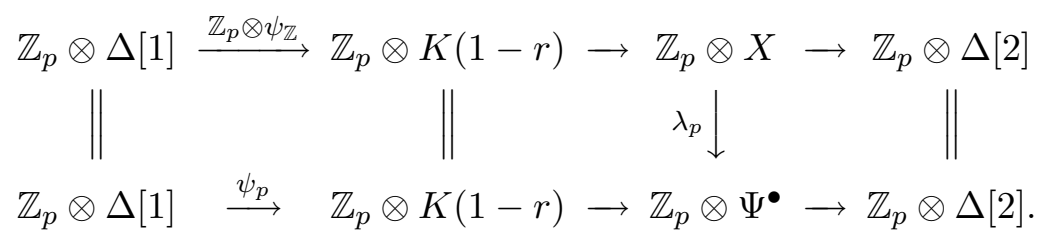

Here the upper row is the scalar extension of (52) and the lower row is the exact triangle induced by property (a) and a choice of isomorphism $\theta_{p}: C_{p, r}^{\bullet} \cong \mathbb{Z}_{p} \otimes \Psi^{\bullet}$ in $D\left(\mathbb{Z}_{p}[G]\right)$ for which, for both $a=0,1$, the composite $\left(\mathbb{Z}_{p} \otimes \iota_{a}\right) \circ H^{a}\left(\theta_{p}\right)$ is equal to the inverse of the isomorphism $\mu_{p, a}^{\prime}$ in (49). (Note that the existence of such an isomorphism $\theta_{p}$ is an easy consequence of the definition of $\epsilon_{\tau_{r}}$.) Now the commutative diagram of $[22,(115)]$ combines with the above diagram to imply 
that the criterion of Theorem 10.1.1(i) is satisfied if one takes $\mathfrak{A}=\mathbb{Z}[G], C_{1}^{\bullet}=$ $X, C_{2}^{\bullet}=\Psi^{\bullet}$ and $\psi_{p}=\lambda_{p}$ for all $p$ and so by Theorem 10.1.1(i), (iii) there exists an isomorphism $X \cong \Psi^{\bullet}$ in $D(\mathbb{Z}[G])$ and hence also an exact triangle in $D^{\text {perf }}(\mathbb{Z}[G])$ of the form $\Delta[1] \rightarrow K(1-r) \rightarrow \Psi^{\bullet} \rightarrow \Delta[2]$. By the additivity of Euler characteristics on exact triangles of perfect complexes one therefore has

$$
\chi\left(\Psi^{\bullet}\right)=\chi(K(1-r))-\chi(\Delta[1])=\Omega_{-r}(K / k)+\left(\mathbb{Z} \Sigma_{\infty}\right)+\chi(\Delta)=\Omega_{-r}(K / k)
$$

where the second equality is by (51) and the third by the description of $\Delta$ given in (c) above. Since, by definition, $\chi\left(\epsilon_{\tau_{r}}\right):=\chi\left(\Psi^{\bullet}\right)$ we thus deduce the required equality $\chi\left(\epsilon_{\tau_{r}}\right)=\Omega_{-r}(K / k)$.

11.3. Justifying Remark 6.2.4. At this stage it only remains to prove that, as claimed in Remark 6.2.4, the equality (17) with $\tau=\tau_{r}$ refines [33, Conj. 6.12]. (We recall that this observation answers the question raised by Chinburg et al in [33, top of p. 357].) To do this we set $\Lambda:=\mathbb{Z}\left[\frac{1}{2}\right]$ and $X_{\Lambda}:=\Lambda \otimes X$ for any module $X$ and use the notation of [33] (but with ' $N$ ' and ' $r$ ' of loc. cit. replaced by $K$ and $1-r$ respectively). Then [33, Prop. 3.9] implies that the map $\mathrm{ch}_{1}$ induces an isomorphism $K_{1-2 r}\left(\mathcal{O}_{K, S}\right)_{\Lambda} \cong\left(K_{1-2 r}^{*} \cap \operatorname{ker}\left(d_{1}\right)\right)_{\Lambda}$. Also each of the modules $Y_{3-2 r, \Lambda}, \operatorname{Div}_{\mathbb{Z}}\left(\Sigma_{\infty}\right)_{\Lambda}$ and $Y_{1-2 r, \Lambda}$ which occur in [33] are projective $\Lambda[G]$-modules. Hence, for each character $\psi \in \operatorname{Irr}(G)$, the diagram of [33, Cor. 6.3] induces a commutative diagram of $\Lambda \otimes \mathcal{O}$-modules of the form

$$
\begin{aligned}
& 0 \rightarrow\left(Y_{3-2 r, \Lambda}\right)_{\psi} \rightarrow\left(\operatorname{Div}_{\mathbb{Z}}\left(\Sigma_{\infty}\right)_{\Lambda}\right)_{\psi} \rightarrow\left(Y_{1-2 r, \Lambda}\right)_{\psi} \quad \rightarrow 0 \\
& \phi_{Y}^{\psi} \circ t_{1} \downarrow \quad\left(\phi^{\prime}\right)^{\psi} \circ t_{2} \downarrow \quad \phi^{\psi} \circ t_{3} \downarrow \\
& 0 \rightarrow Y_{3-2 r, \Lambda}^{\psi} \rightarrow\left(K_{1-2 r, \Lambda}^{\prime}\right)^{\psi} \rightarrow K_{1-2 r}\left(\mathcal{O}_{K, S}\right)_{\Lambda}^{\psi} \rightarrow 0
\end{aligned}
$$

in which $t_{1}, t_{2}$ and $t_{3}$ denote $t\left(Y_{3-2 r, \Lambda}, \psi\right), t\left(\operatorname{Div}_{\mathbb{Z}}\left(\Sigma_{\infty}\right)_{\Lambda}, \psi\right)$ and $t\left(Y_{1-2 r, \Lambda}, \psi\right)$ in the notation of (16), and each vertical arrow in the diagram is injective (and thus has finite cokernel). We write $\varphi$ for the composite $H_{\tau_{r}, \Lambda}^{1} \rightarrow\left(H_{\tau_{r}, \mathrm{tf}}^{1}\right)_{\Lambda}=H_{r, \Lambda}^{+} \rightarrow$ $Y_{1-2 r, \Lambda} \rightarrow K_{1-2 r}\left(\mathcal{O}_{K, S}\right)_{\Lambda}$ where the first arrow is the tautological projection, the second is the isomorphism induced by multiplication by $(2 \pi i)^{r}$ and the third is induced by the map $\phi$ in diagram [33, (6.6)]. Then by applying the Snake lemma to the above diagram and taking account of both [33, (6.10)] (with $V=V_{\psi}$ ) and the equality $K_{-2 r}\left(\mathcal{O}_{K, S}\right)_{\Lambda}=K_{-2 r, \Lambda}^{\prime}$ one finds that the ideal $\left(A_{\varphi}^{\tau_{r}}(\psi) q_{\varphi}^{\tau_{r}}(\psi)^{-1}\right)_{\Lambda}$ which occurs in (17) is equal to the image under $\Lambda \otimes-$ of the ideal $A\left(V_{\psi}, \phi^{\prime}\right) q_{T_{\psi}}\left(\phi^{\prime}\right)^{-1}$ which occurs in [33, Conj. 6.12]. Hence, from the conjectural equality (17) with $\tau=\tau_{r}$, we deduce that the ideal $E\left(V_{\psi}, \phi^{\prime}\right) \cdot \mathcal{O}$ in [33, Conj. 6.12] should be 
supported entirely above 2. It is clear that this refinement of [33, Conj. 6.12] is consistent with the result of [33, Cor. 6.16].

\section{Remark 11.3.1. Generalisations}

Let $M$ be any motive defined over $k$ for which both of the spaces $H^{0}(k, M)$ and $H^{0}\left(k, M^{*}(1)\right)$ vanish. Then for every prime $p$, every full Galois stable $\mathbb{Z}_{p^{-}}$ sublattice $T_{p}$ of $H_{p}(M)$ and every large enough finite set of places $S$ of $k$ it can be shown that the complex $R \operatorname{Hom}_{\mathbb{Z}_{p}}\left(R \Gamma_{c}\left(\mathcal{O}_{k, S}\left[\frac{1}{p}\right], \mathbb{Z}_{p}[G] \otimes_{\mathbb{Z}_{p}} T_{p}\right), \mathbb{Z}_{p}[-2]\right)$ belongs to $D^{\text {perf }}\left(\mathbb{Z}_{p}[G]\right)$ and is acyclic outside degrees 0 and 1 . If now $M$ has trivial tangent space (that is, $H_{\mathrm{dR}}(M)=F^{0} H_{\mathrm{dR}}(M)$ ) and also satisfies certain standard expectations (including the 'Coherence hypothesis' of [23, p. 525]), then it is possible to mimic the construction of $\tau_{r}$ given above to obtain an a.t.e. of $\mathbb{Z}[G]$-modules $\tau_{M}$ with the property that $\operatorname{ETNC}\left(M_{K}, \mathbb{Z}[G]\right)$ is valid if and only if $\chi\left(\tau_{M}\right)$ vanishes.

\section{The proof of Proposition 4.3.1}

Throughout this section we assume the notation and hypotheses of Proposition 4.3.1. To prove this result we adapt the argument given in $[21, \S 1.5 .1, \S 1.7]$.

For convenience, we shall therefore adopt the notation of $[21, \S 1.5]$. In particular, if $q$ is any odd prime which does not divide $\operatorname{disc}(K) \operatorname{cond}(E)$, then we define a finitely generated (free) $\mathbb{Z}_{q}[G]$-module by setting $\mathcal{D}_{q}:=D_{\mathrm{cr}, q}\left(T_{q}\right) \cong$ $\mathcal{O}_{K, q} \otimes_{\mathbb{Z}_{q}} D_{\text {cr }, q}\left(T_{q}(E)\right)$ where $\mathcal{O}_{K, q}:=\mathbb{Z}_{q} \otimes \mathcal{O}_{K}$ and $D_{\text {cr }, q}$ is the quasi-inverse to the functor of Fontaine and Lafaille that is used by Niziol in [80]. For each such $q$ we also write $\phi_{q}$ for the natural $\left(\mathbb{Z}_{q}[G]\right.$-equivariant) Frobenius on $\mathcal{D}_{q}$.

We write $S_{\text {ram }}$ and $S_{\text {bad }}$ for the (finite) sets of primes which divide $\operatorname{disc}(K)$ and $\operatorname{cond}(E)$ respectively and also define $S:=S_{\text {ram }} \cup S_{\text {bad }}$.

12.1. Tamagawa factors. We first define the Tamagawa factors of the pair $\left(h^{1}\left(E_{/ F}\right)(1), \mathbb{Z}[\operatorname{Gal}(F / \mathbb{Q})]\right)$ which occur in Proposition 4.3.1.

For each prime $q$ we write $T_{q}(E)$ for the $q$-adic Tate module of $E$ and for each finite degree Galois extension $F$ of $\mathbb{Q}$ we set $T_{q, F}:=\mathbb{Z}_{q}[\operatorname{Gal}(F / \mathbb{Q})] \otimes_{\mathbb{Z}_{q}} T_{q}(E)$ which we regard as a (left) module over $G_{\mathbb{Q}} \times \operatorname{Gal}(F / \mathbb{Q})$ in the natural way. For each prime $\ell$ we write $\mathbb{Q}_{\ell}^{\text {un }}$ for the maximal unramified extension of $\mathbb{Q}_{\ell}$ in a fixed 
algebraic closure $\mathbb{Q}_{\ell}^{c}$ and set $I_{\ell}:=\operatorname{Gal}\left(\mathbb{Q}_{\ell}^{c} / \mathbb{Q}_{\ell}^{\text {un }}\right)$. We then consider the group

$$
C_{\ell}(E, F):=\bigoplus_{q} H^{0}\left(\mathbb{Q}_{\ell}, H^{1}\left(I_{\ell}, T_{q, F}\right)_{\text {tor }}\right),
$$

where in the direct sum $q$ runs over all primes. This group is finite: indeed, each summand is clearly finite and it is known that almost all summands are isomorphic to a subgroup of the direct sum over all characteristic $\ell$ residue fields $\kappa$ of $F$ of the (finite) group of connected components of the special fibre of a Néron model of $E_{/ F}$ over $\kappa$ (cf. [57, Exp. IX, (11.3.8)]). We may therefore define the Tamagawa factor at $\ell$ of the pair $\left(h^{1}\left(E_{/ F}\right)(1), \mathbb{Z}[\operatorname{Gal}(F / \mathbb{Q})]\right)$ by setting

$$
c_{\ell}(E, F):=\left|C_{\ell}(E, F)\right| \text {. }
$$

12.2. Cohomology with finite support. If $F=K$, then in the sequel we shall always abbreviate $T_{q, F}$ to $T_{q}$ and set $V_{q}=\mathbb{Q}_{q} \otimes_{\mathbb{Z}_{q}} T_{q}$ (again regarded as a (left) module over $G_{\mathbb{Q}} \times G$ in the natural way).

For each pair of primes $\ell$ and $q$ we write $H_{f}^{1}\left(\mathbb{Q}_{\ell}, T_{q}\right)_{\mathrm{BK}}$ for the finite support cohomology group defined by Bloch and Kato in [9]. If $q$ is any prime which does not divide $|G|$ we then define for each prime $\ell$ a complex of $\mathbb{Z}_{q}[G]$-modules by setting

$$
R \Gamma_{f}\left(\mathbb{Q}_{\ell}, T_{q}\right):=H_{f}^{1}\left(\mathbb{Q}_{\ell}, T_{q}\right)_{\mathrm{BK}}[-1] .
$$

If condition (B) of Proposition 4.3.1 is satisfied, in which case $|G|$ is a power of a prime $p$, then for each prime $\ell$ we also define a complex of $\mathbb{Z}_{p}[G]$-modules by setting

$$
R \Gamma_{f}\left(\mathbb{Q}_{\ell}, T_{p}\right):= \begin{cases}T_{p}^{I_{\ell}} \stackrel{1-\mathrm{Fr}_{\ell}^{-1}}{\longrightarrow} T_{p}^{I_{\ell}}, & \text { if } \ell \neq p \\ F^{0} \mathcal{D}_{p} \stackrel{1-\phi_{p}^{0}}{\longrightarrow} \mathcal{D}_{p}, & \text { if } \ell=p\end{cases}
$$

where $\operatorname{Fr}_{\ell}$ is the natural Frobenius in $\operatorname{Gal}\left(\mathbb{Q}_{\ell}^{\text {un }} / \mathbb{Q}_{\ell}\right), \phi_{p}^{0}$ is the restriction of $\phi_{p}$ to $F^{0} \mathcal{D}_{p} \subseteq \mathcal{D}_{p}$ and in both cases the first term is placed in degree 0 . (Note that in defining $R \Gamma_{f}\left(\mathbb{Q}_{p}, T_{p}\right)$ in this way we are using the conditions (B)(ii) and (B)(iv). For further observations regarding our definitions of the complexes $R \Gamma_{f}\left(\mathbb{Q}_{\ell}, T_{q}\right)$ see Remark 12.4.2 below.)

We fix a place $w$ of $K$ above $\ell$ and write $G_{w}$ for the decomposition subgroup of $w$ in $G$ (which we identify with $\operatorname{Gal}\left(K_{w} / \mathbb{Q}_{\ell}\right)$ ) and $\overline{I_{w}}$ for the image of $I_{\ell}$ in $G_{w}$.

Lemma 12.2.1. For all primes $\ell$ and $q$ the complex $R \Gamma_{f}\left(\mathbb{Q}_{\ell}, T_{q}\right)$ belongs to $D^{\text {perf }}\left(\mathbb{Z}_{q}[G]\right)$. 
Proof. If $q \nmid|G|$, then the algebra $\mathbb{Z}_{q}[G]$ is regular and so $R \Gamma_{f}\left(\mathbb{Q}_{\ell}, T_{q}\right)$ belongs to $D^{\text {perf }}\left(\mathbb{Z}_{q}[G]\right)$ simply because $H_{f}^{1}\left(\mathbb{Q}_{\ell}, T_{q}\right)_{\mathrm{BK}}$ is a finitely generated $\mathbb{Z}_{q}[G]$-module.

We shall therefore assume that condition (B) is satisfied and restrict to the case $q=p$. To deal with the case $\ell=p$ we note that $\mathcal{O}_{K, p}$ is a free $\mathbb{Z}_{p}[G]$-module (as $p$ is unramified in $K / \mathbb{Q}$ by condition (B)(ii)). Thus both $F^{0} \mathcal{D}_{p} \cong \mathcal{O}_{K, p} \otimes_{\mathbb{Z}_{p}}$ $F^{0} D_{\text {cr }, p}\left(T_{p}(E)\right)$ and $\mathcal{D}_{p} \cong \mathcal{O}_{K, p} \otimes_{\mathbb{Z}_{p}} D_{\text {cr }, p}\left(T_{p}(E)\right)$ are finitely generated $\mathbb{Z}_{p}[G]$ modules that have finite projective dimension and so it is clear that $R \Gamma_{f}\left(\mathbb{Q}_{p}, T_{p}\right)$ belongs to $D^{\text {perf }}\left(\mathbb{Z}_{p}[G]\right)$.

If $\ell \neq p$ and $\ell \notin S_{\text {ram }}$, then $T_{p}^{I_{\ell}}=\mathbb{Z}_{p}[G] \otimes_{\mathbb{Z}_{p}} T_{p}(E)^{I_{\ell}}$ is a free $\mathbb{Z}_{p}[G]$-module and so the result is also clear. It thus suffices to prove that if $(\ell \neq p$ and $) \ell \in S_{\text {ram }}$, then $R \Gamma_{f}\left(\mathbb{Q}_{\ell}, T_{p}\right)$ is acyclic. To do this we let $d_{\ell, p}^{0}$ denote the differential (in degree $0)$ of $R \Gamma_{f}\left(\mathbb{Q}_{\ell}, T_{p}\right)$. Then $\operatorname{ker}\left(d_{\ell, p}^{0}\right)=T_{p}^{\mathrm{Gal}\left(\mathbb{Q}_{\ell}^{c} / \mathbb{Q}_{\ell}\right)}$ vanishes and hence it is enough to show that $\operatorname{cok}\left(d_{\ell, p}^{0}\right)$ vanishes or equivalently, since both $G$ and $\operatorname{cok}\left(d_{\ell, p}^{0}\right)$ are finite $p$-groups, that $\operatorname{cok}\left(d_{\ell, p}^{0}\right)_{G}$ vanishes. Now condition (B)(ii) implies that $E$ has good reduction at $\ell$ and so $T_{p}^{I_{\ell}}=\mathbb{Z}_{p}\left[G / \overline{I_{w}}\right] \otimes_{\mathbb{Z}_{p}} T_{p}(E)$. The (finite) $\mathbb{Z}_{p}$-module $\operatorname{cok}\left(d_{\ell, p}^{0}\right)_{G}$ is therefore isomorphic to the cokernel of the (injective) endomorphism of the (free) $\mathbb{Z}_{p}$-module $T_{p}(E)$ that is induced by the action of $1-\mathrm{Fr}_{\ell}^{-1}$ and so $\left|\operatorname{cok}\left(d_{\ell, p}^{0}\right)_{G}\right|$ is equal to the maximum power of $p$ which $\operatorname{divides} \operatorname{det}_{\mathbb{Z}_{p}}\left(1-\operatorname{Fr}_{\ell}^{-1} \mid\right.$ $\left.T_{p}(E)\right)$ in $\mathbb{Z}_{p}$. But the latter determinant is equal to the value at $t=1$ of the Euler factor $1-\left(\ell+1-\left|\tilde{E}_{\ell}\left(\mathbb{F}_{\ell}\right)\right|\right) t+\ell t^{2}$ and this value is $\left|\tilde{E}_{\ell}\left(\mathbb{F}_{\ell}\right)\right|$ which, by condition $(\mathrm{B})(\mathrm{iii})$, is a unit in $\mathbb{Z}_{p}$.

For all primes $q$ and $\ell$ we write $R \Gamma\left(\mathbb{Z}_{S}\left[\frac{1}{q}\right], T_{q}\right)$ and $R \Gamma\left(\mathbb{Q}_{\ell}, T_{q}\right)$ for the usual complexes of continuous cochains. Then if either $q \nmid|G|$ or $q=p$ and condition (B) is satisfied, the argument of $[21, \S 1.5 .1]$ gives a natural morphism $\varpi_{\ell, q}: R \Gamma_{f}\left(\mathbb{Q}_{\ell}, T_{q}\right) \rightarrow R \Gamma\left(\mathbb{Q}_{\ell}, T_{q}\right)$ in $D\left(\mathbb{Z}_{q}[G]\right)$. Following [21, (1.33)], we define $R \Gamma_{/ f}\left(\mathbb{Q}_{\ell}, T_{q}\right)$ to be a complex of $\mathbb{Z}_{q}[G]$-modules which lies in an exact triangle in $D\left(\mathbb{Z}_{q}[G]\right)$ of the form

$$
R \Gamma_{f}\left(\mathbb{Q}_{\ell}, T_{q}\right) \stackrel{\varpi_{\ell, q}}{\longrightarrow} R \Gamma\left(\mathbb{Q}_{\ell}, T_{q}\right) \stackrel{\varpi_{\ell, q}^{\prime}}{\longrightarrow} R \Gamma_{/ f}\left(\mathbb{Q}_{\ell}, T_{q}\right) \rightarrow R \Gamma_{f}\left(\mathbb{Q}_{\ell}, T_{q}\right)[1] .
$$

We then define the complex $R \Gamma_{f}\left(\mathbb{Q}, T_{q}\right)$ so that it lies in an exact triangle in $D\left(\mathbb{Z}_{q}[G]\right)$ of the form

$$
R \Gamma_{f}\left(\mathbb{Q}, T_{q}\right) \rightarrow R \Gamma\left(\mathbb{Z}_{S}\left[\frac{1}{q}\right], T_{q}\right) \stackrel{\theta}{\rightarrow} \bigoplus_{\ell \in S \cup\{q\}} R \Gamma_{/ f}\left(\mathbb{Q}_{\ell}, T_{q}\right) \rightarrow R \Gamma_{f}\left(\mathbb{Q}, T_{q}\right)[1]
$$


where the $\ell$-component of $\theta$ is equal to the composite of the natural localisation morphism $R \Gamma\left(\mathbb{Z}_{S}\left[\frac{1}{q}\right], T_{q}\right) \rightarrow R \Gamma\left(\mathbb{Q}_{\ell}, T_{q}\right)$ and the morphism $\varpi_{\ell, q}^{\prime}$.

In each degree $i$ we set $H_{f}^{i}\left(\mathbb{Q}, T_{q}\right):=H^{i}\left(R \Gamma_{f}\left(\mathbb{Q}, T_{q}\right)\right)$.

Lemma 12.2.2. If either $q \nmid|G|$ or condition (B) is satisfied, then the complex $R \Gamma_{f}\left(\mathbb{Q}, T_{q}\right)$ belongs to $D^{\text {perf }}\left(\mathbb{Z}_{q}[G]\right)$. Further, it is acyclic outside degrees 1,2 and 3 and there are canonical isomorphisms

$$
H_{f}^{i}\left(\mathbb{Q}, T_{q}\right) \cong \begin{cases}\mathbb{Z}_{q} \otimes E(K), & \text { if } i=1, \\ \mathbb{Z}_{q} \otimes \operatorname{Sel}\left(E_{/ K}\right), & \text { if } i=2, \\ \mathbb{Z}_{q} \otimes\left(E(K)_{\text {tor }}\right)^{\vee}, & \text { if } i=3 .\end{cases}
$$

Proof. We first consider the cohomology modules. In fact, if either $i \notin\{1,2\}$ or $q \nmid|G|$, then the description of $H_{f}^{i}\left(\mathbb{Q}, T_{q}\right)$ given above follows immediately from the argument of $[21$, p. 86-87]. For the remaining case that condition (B) is satisfied, $q=p$ and $i \in\{1,2\}$ the argument of loc. cit. gives a canonical exact sequence of $\mathbb{Z}_{p}[G]$-modules

$$
\begin{aligned}
0 \rightarrow H_{f}^{1}\left(\mathbb{Q}, T_{p}\right) \rightarrow \mathbb{Z}_{p} \otimes E(K) \rightarrow \bigoplus_{\ell \in S} H^{0}\left(\mathbb{Q}_{\ell}, H^{1}\left(I_{\ell}, T_{p}\right)_{\text {tor }}\right) & \\
& \rightarrow H_{f}^{2}\left(\mathbb{Q}, T_{p}\right) \rightarrow \mathbb{Z}_{p} \otimes \operatorname{Sel}\left(E_{/ K}\right) \rightarrow 0
\end{aligned}
$$

and so we must show that $H^{0}\left(\mathbb{Q}_{\ell}, H^{1}\left(I_{\ell}, T_{p}\right)_{\text {tor }}\right)=0$ for all $\ell$ in $S$. The natural isomorphism

$$
H^{0}\left(\mathbb{Q}_{\ell}, H^{1}\left(I_{\ell}, T_{p}\right)_{\text {tor }}\right) \cong \mathbb{Z}_{p}[G] \otimes_{\mathbb{Z}_{p}\left[G_{w}\right]} H^{0}\left(K_{w}^{\overline{I_{w}}}, H^{1}\left(K_{w} \mathbb{Q}_{\ell}^{\text {un }}, T_{p}(E)\right)_{\text {tor }}\right)
$$

therefore implies that we must prove that $H^{0}\left(K_{w}^{\overline{I_{w}}}, H^{1}\left(K_{w} \mathbb{Q}_{\ell}^{\text {un }}, T_{p}(E)\right)_{\text {tor }}\right)$ vanishes for all $\ell$ in $S$. Now if $\ell \notin S_{\text {bad }}$, then the group $H^{1}\left(K_{w} \mathbb{Q}_{\ell}^{\text {un }}, T_{p}(E)\right)=$ $\operatorname{Hom}_{\text {cont }}\left(\operatorname{Gal}\left(\mathbb{Q}_{\ell}^{c} / K_{w} \mathbb{Q}_{\ell}^{\text {un }}\right), T_{p}(E)\right)$ is torsion-free and so the result is clear. On the other hand, if $\ell \in S_{\text {bad }}$, then condition (B)(ii) implies that $\ell \notin S_{\text {ram }}$. This implies $K_{w}^{\overline{I_{w}}}=K_{w}$ and $K_{w} \mathbb{Q}_{\ell}^{\text {un }}=\mathbb{Q}_{\ell}^{\text {un }}$ and hence we must show that $H^{0}\left(K_{w}, H^{1}\left(I_{\ell}, T_{p}(E)\right)_{\text {tor }}\right)=0$. But $G_{w}$ is a $p$-group and so this is true because $H^{0}\left(K_{w}, H^{1}\left(I_{\ell}, T_{p}(E)\right)_{\text {tor }}\right)^{G_{w}}=H^{0}\left(\mathbb{Q}_{\ell}, H^{1}\left(I_{\ell}, T_{p}(E)\right)_{\text {tor }}\right)=0$, where the last equality follows from condition $(\mathrm{B})(\mathrm{v})$ (and the definition of $c_{\ell}(E, \mathbb{Q})$ ).

At this stage it suffices to prove that $R \Gamma_{f}\left(\mathbb{Q}, T_{q}\right)$ belongs to $D^{\text {perf }}\left(\mathbb{Z}_{q}[G]\right)$. If $q \nmid|G|$, then $\mathbb{Z}_{q}[G]$ is regular and so $R \Gamma_{f}\left(\mathbb{Q}, T_{q}\right)$ belongs to $D^{\text {perf }}\left(\mathbb{Z}_{q}[G]\right)$ by virtue of the fact that each module $H_{f}^{i}\left(\mathbb{Q}, T_{q}\right)$ is a finitely generated $\mathbb{Z}_{q}[G]$-module (which 
is zero for almost all $i$ ). Lastly, if $q=p$ and condition (B) is satisfied, then $(q$ is odd so) each complex $R \Gamma\left(\mathbb{Z}_{S}\left[\frac{1}{q}\right], T_{q}\right)$ and $R \Gamma\left(\mathbb{Q}_{\ell}, T_{q}\right)$ belongs to $D^{\text {perf }}\left(\mathbb{Z}_{q}[G]\right)$ (see, for example, [44]) and so Lemma 12.2.1 combines with the exact triangles (53) and (54) to imply that the same is true of $R \Gamma_{f}\left(\mathbb{Q}, T_{q}\right)$.

12.3. An auxiliary a.t.e. We assume first that condition (B) of Proposition 4.3.1 is satisfied. Then $G$ is a $p$-group and so, if $p \in \operatorname{Spec}(\Lambda)$, then Lemma 12.2.2 combines with (48) to give a canonical isomorphism

$$
\operatorname{Ext}_{\Lambda[G]}^{2}\left(\Lambda \otimes \operatorname{Sel}\left(E_{/ K}\right), \Lambda \otimes E(K)\right) \cong \operatorname{Ext}_{\mathbb{Z}_{p}[G]}^{2}\left(H_{f}^{2}\left(\mathbb{Q}, T_{p}\right), H_{f}^{1}\left(\mathbb{Q}, T_{p}\right)\right) .
$$

Now the cardinality of $E(\mathbb{Q})_{\text {tor }}=E(K)_{\text {tor }}^{G}$ is prime to $p$ by condition (B)(iii). Hence (since $G$ is a $p$-group) the cardinality of $E(K)_{\text {tor }}$ is also prime to $p$ and so Lemma 12.2.2 implies that $R \Gamma_{f}\left(\mathbb{Q}, T_{p}\right)$ is both perfect and acyclic outside degrees 1 and 2. We may therefore define an a.t.e. of $\Lambda[G]$-modules $\tau^{\prime}$ with the following properties:-

(a) $H_{\tau^{\prime}}^{0}=(\Lambda \otimes E(K)) \oplus\left(\Lambda \otimes\left(E(K)_{\text {tor }}\right)^{\vee}\right)$ and $H_{\tau^{\prime}}^{1}=\Lambda \otimes \operatorname{Sel}\left(E_{/ K}\right)$;

(b) $\epsilon_{\tau^{\prime}}$ is the element of

$$
\operatorname{Ext}_{\Lambda[G]}^{2}\left(H_{\tau^{\prime}}^{1}, H_{\tau^{\prime}}^{0}\right)=\operatorname{Ext}_{\Lambda[G]}^{2}\left(\Lambda \otimes \operatorname{Sel}\left(E_{/ K}\right), \Lambda \otimes E(K)\right)
$$

that corresponds under (55) to the shifted complex $R \Gamma_{f}\left(\mathbb{Q}, T_{p}\right)[1]$;

(c) $\lambda_{\tau^{\prime}}$ is induced by the Néron-Tate height pairing;

(d) $\left(\mathcal{L}_{\tau^{\prime}}^{*}\right)_{\psi}=L^{*}(E, \bar{\psi}, 1)$ for each $\psi \in \operatorname{Irr}(G)$.

If on the other hand either condition (B) is satisfied and $p$ is invertible in $\Lambda$ or condition (A) of Proposition 4.3.1 is satisfied, then $|G|$ is invertible in $\Lambda$ and so all groups of the form $\operatorname{Ext}_{\Lambda[G]}^{2}(-,-)$ vanish. In these cases therefore we simply let $\tau^{\prime}$ be the unique a.t.e. of $\Lambda[G]$-modules which satisfies the conditions (a), (c) and (d) above.

We also set $M:=h^{1}\left(E_{/ K}\right)(1)$ and $t_{\mathrm{dR}}(M):=H^{1}\left(E_{/ K}, \mathcal{O}_{E_{/ K}}\right) \cong H_{\mathrm{dR}}^{0}(M) / F^{0}$ and define a free rank one $\Lambda[G]$-module by setting

$$
\begin{aligned}
H_{B, \Lambda}^{+}:=\Lambda[G] \otimes H^{0} & \left(\operatorname{Gal}(\mathbb{C} / \mathbb{R}), \operatorname{Hom}_{\mathbb{Z}}\left(H_{1}(E(\mathbb{C}), \mathbb{Z}), 2 \pi i \mathbb{Z}\right)\right) \\
& \cong H^{0}\left(\operatorname{Gal}(\mathbb{C} / \mathbb{R}), \operatorname{Hom}_{\mathbb{Z}}\left(\bigoplus_{\sigma: K \rightarrow \mathbb{C}} H_{1}\left(\sigma E_{/ K}(\mathbb{C}), \mathbb{Z}\right), 2 \pi i \Lambda\right)\right),
\end{aligned}
$$

where the last isomorphism follows from the fact that $K$ is assumed to be totally real. We also write $\mathcal{E}_{K}$ for the Néron model of $E$ over $\mathcal{O}_{K}$. Then $\Lambda \otimes H^{1}\left(\mathcal{E}_{K}, \mathcal{O}_{\mathcal{E}_{K}}\right)$ 
is a full sublattice of $t_{\mathrm{dR}}(M)$ which is also a projective $\Lambda[G]$-module (see Lemma 12.5.1 below) and so we may define an element of $K_{0}(\Lambda[G], \mathbb{R}[G])$ by setting

$$
\xi:=\chi\left(\tau^{\prime}\right)+T \Omega(M, \Lambda[G])+\left(H_{B, \Lambda}^{+}, \Lambda \otimes H^{1}\left(\mathcal{E}_{K}, \mathcal{O}_{\mathcal{E}_{K}}\right) ; \pi\right)-\sum_{\ell \in S_{\mathrm{ram}}} \epsilon_{\ell}
$$

Here $\pi: \mathbb{R} \otimes_{\Lambda} H_{B, \Lambda}^{+} \cong \mathbb{R} \otimes_{\mathbb{Q}} t_{\mathrm{dR}}(M)$ is the canonical period isomorphism described by Deligne in $[38, \S 4.1]$ and for each prime $q$ we set

$$
\epsilon_{q}:=\delta_{\Lambda}\left(L_{q}(\mathbb{Q}[G] M, 0)\right) \in K_{0}(\Lambda[G], \mathbb{Q}[G]),
$$

where $L_{q}(\mathbb{Q}[G] M, 0) \in \zeta(\mathbb{Q}[G])^{\times}$is the value at $s=0$ of the equivariant local $L$-factor $L_{q}(\mathbb{Q}[G] M, s)$ defined in $[23, \S 4.1$, Rem. 7].

Lemma 12.3.1. $\xi$ belongs to the subgroup $K_{0}(\Lambda[G], \mathbb{Q}[G])$ of $K_{0}(\Lambda[G], \mathbb{R}[G])$.

Proof. We write $R \Omega(M, \Lambda[G])$ for the image in $K_{0}(\Lambda[G], \mathbb{R}[G])$ of the element $R \Omega(M, \mathbb{Z}[G])$ of $K_{0}(\mathbb{Z}[G], \mathbb{R}[G])$ that is defined in $[23, \S 3.4$, just before Lem. 7]. Then $T \Omega(M, \Lambda[G])$ is, by definition, equal to $R \Omega(M, \Lambda[G])+\delta_{\Lambda}\left(\mathcal{L}_{\tau^{\prime}}^{*}\right)$. Hence, since $\chi\left(\tau^{\prime}\right):=\left[\epsilon_{\tau^{\prime}}, \lambda_{\tau^{\prime}}\right]-\delta_{\Lambda}\left(\mathcal{L}_{\tau^{\prime}}^{*}\right)$ and each $\epsilon_{\ell}$ belongs to $K_{0}(\Lambda[G], \mathbb{Q}[G])$ it is enough to show that the sum $\left[\epsilon_{\tau^{\prime}}, \lambda_{\tau^{\prime}}\right]+\left(H_{B, \Lambda}^{+}, \Lambda \otimes H^{1}\left(\mathcal{E}_{K}, \mathcal{O}_{\mathcal{E}_{K}}\right) ; \pi\right)+R \Omega(M, \Lambda[G])$ belongs to $K_{0}(\Lambda[G], \mathbb{Q}[G])$. It is therefore enough to show that the images under the natural map $K_{0}(\Lambda[G], \mathbb{R}[G]) \rightarrow K_{0}(\mathbb{Q}[G], \mathbb{R}[G])$ of the elements $\left[\epsilon_{\tau^{\prime}}, \lambda_{\tau^{\prime}}\right]+$ $\left(H_{B, \Lambda}^{+}, \Lambda \otimes H^{1}\left(\mathcal{E}_{K}, \mathcal{O}_{\mathcal{E}_{K}}\right) ; \pi\right)$ and $-R \Omega(M, \Lambda[G])$ coincide. But, unwinding definitions, it is easily seen that the image of both of these elements is equal to $\left(\mathbb{Q} \otimes E(K), \mathbb{Q} \otimes E(K)^{*} ; \iota\right)+\left(\mathbb{Q} \otimes_{\Lambda} H_{B, \Lambda}^{+}, t_{\mathrm{dR}}(M) ; \pi\right)$ where $\iota$ is induced by the Néron-Tate height pairing.

We recall (from, for example, $[23,(13)]$ ) that there is a natural direct sum decomposition of abelian groups

$$
K_{0}(\Lambda[G], \mathbb{Q}[G])=\bigoplus_{\ell \in \operatorname{Spec}(\Lambda)} K_{0}\left(\mathbb{Z}_{\ell}[G], \mathbb{Q}_{\ell}[G]\right)
$$

Following Lemma 12.3.1 we may therefore define for each prime $\ell \in \operatorname{Spec}(\Lambda)$ an element $\xi_{\ell}$ of $K_{0}\left(\mathbb{Z}_{\ell}[G], \mathbb{Q}_{\ell}[G]\right)$ by setting

$$
\xi=\sum_{\ell \in \operatorname{Spec}(\Lambda)} \xi_{\ell} .
$$


12.4. Computation of the elements $\xi_{\ell}$. For each prime $q$ we write $t_{q}\left(V_{q}\right)$ for the tangent space $\operatorname{DR}_{q}\left(V_{q}\right) / F^{0}$ of $V_{q}$ and $\kappa_{q}: \mathbb{Q}_{q} \otimes_{\mathbb{Q}} t_{\mathrm{dR}}(M) \cong t_{q}\left(V_{q}\right)$ for the canonical comparison isomorphism. We also define a space $H_{f}^{1}\left(\mathbb{Q}_{q}, V_{q}\right):=\mathbb{Q}_{q} \otimes_{\mathbb{Z}_{q}}$ $H_{f}^{1}\left(\mathbb{Q}_{q}, T_{q}\right)_{\text {BK }}$ and write $\exp _{q}^{\mathrm{BK}}: t_{q}\left(V_{q}\right) \rightarrow H_{f}^{1}\left(\mathbb{Q}_{q}, V_{q}\right)$ for the isomorphism given by the Bloch-Kato exponential map. For each embedding $j: \mathbb{R} \rightarrow \mathbb{C}_{q}$ we write $\pi_{j}: \mathbb{C}_{q} \otimes_{\Lambda} H_{B, \Lambda}^{+} \cong \mathbb{C}_{q} \otimes_{\mathbb{Q}_{q}} t_{q}\left(V_{q}\right)$ for the composite $\left(\mathbb{C}_{q} \otimes_{\mathbb{Q}_{q}} \kappa_{q}\right) \circ\left(\mathbb{C}_{q} \otimes_{\mathbb{R}, j} \pi\right)$. We also set $\mathcal{J}_{q}:=\kappa_{q}\left(\mathbb{Z}_{q} \otimes H^{1}\left(\mathcal{E}_{K}, \mathcal{O}_{\mathcal{E}_{K}}\right)\right) \subset t_{q}\left(V_{q}\right)$ and let $\mathcal{I}_{q}$ denote the intersection of $\exp _{q}^{\mathrm{BK}}\left(\mathcal{J}_{q}\right)$ with $H_{f}^{1}\left(\mathbb{Q}_{q}, T_{q}\right)_{\mathrm{BK}, \mathrm{tf}} \subset H_{f}^{1}\left(\mathbb{Q}_{q}, V_{q}\right)$.

In order to state the next result we note that if $q \nmid|G|$, then $\mathbb{Z}_{q}[G]$ is a regular ring. Hence for any finitely generated $\mathbb{Z}_{q}[G]$-modules $X$ and $Y$, extension field $F$ of $\mathbb{Q}_{q}$ and isomorphism of $F[G]$-modules $\mu: F \otimes_{\mathbb{Z}_{q}} X \cong F \otimes_{\mathbb{Z}_{q}} Y$ we can define an element $\mathrm{cl}_{q}(X, Y ; \mu):=\left([X][Y]^{-1}, \mu_{\text {triv }}\right)$ of $K_{0}\left(\mathbb{Z}_{q}[G], F[G]\right)$ just as in $\S 6$. In particular, for any finite $\mathbb{Z}_{q}[G]$-module $X$ we may set $\mathrm{cl}_{q}(X):=\operatorname{cl}_{q}(X, 0 ; 0) \in$ $K_{0}\left(\mathbb{Z}_{q}[G], \mathbb{Q}_{q}[G]\right)$.

Lemma 12.4.1. Recall that in $\S 12.3$ we defined for each prime $q$ an element $\epsilon_{q}$ of $K_{0}(\Lambda[G], \mathbb{Q}[G])$ and for each prime $q$ in $\operatorname{Spec}(\Lambda)$ an element $\xi_{q}$ of $K_{0}\left(\mathbb{Z}_{q}[G], \mathbb{Q}_{q}[G]\right)$.

(i) If $q$ does not belong to $S$ and does not divide $2|G|$, then the image of $\epsilon_{q}$ in $K_{0}\left(\mathbb{Z}_{q}[G], \mathbb{Q}_{q}[G]\right)$ is equal to $\operatorname{cl}_{q}\left(\exp _{q}^{\mathrm{BK}}\left(\mathcal{J}_{q}\right) / \mathcal{I}_{q}\right)-\mathrm{cl}_{q}\left(H_{f}^{1}\left(\mathbb{Q}_{q}, T_{q}\right)_{\mathrm{BK}} / \mathcal{I}_{q}\right)$.

(ii) If $q \in \operatorname{Spec}(\Lambda)$ does not divide $|G|$, then $\xi_{q}$ is equal to

$$
\begin{aligned}
& \xi_{q}^{\prime}+\sum_{\ell \in S_{\mathrm{bad}} \backslash\{q\}} \operatorname{cl}_{q}\left(H^{0}\left(\mathbb{Q}_{\ell}, H^{1}\left(I_{\ell}, T_{q}\right)_{\text {tor }}\right)\right)+\sum_{\ell \in S_{\mathrm{ram}} \backslash\{q\}} \operatorname{cl}_{q}\left(\operatorname{cok}\left(1-\mathrm{Fr}_{\ell}^{-1} \mid T_{q}^{I_{\ell}}\right)\right) \\
& \quad \text { with } \xi_{q}^{\prime}=0 \text { if } q \notin S \text { and } \\
& \xi_{q}^{\prime}=\left[\operatorname{cl}_{q}\left(H_{f}^{1}\left(\mathbb{Q}_{q}, T_{q}\right)_{\mathrm{BK}} / \mathcal{I}_{q}\right)+\operatorname{cl}_{q}\left(A_{q}\right)\right]-\left[\operatorname{cl}_{q}\left(\exp _{q}^{\mathrm{BK}}\left(\mathcal{J}_{q}\right) / \mathcal{I}_{q}\right)+\operatorname{cl}_{q}\left(B_{q}\right)\right]
\end{aligned}
$$

if $q \in S$, where $A_{q}$ and $B_{q}$ are finite $\mathbb{Z}_{q}[G]$-modules such that $\operatorname{cl}_{q}\left(A_{q}\right)$ $\mathrm{cl}_{q}\left(B_{q}\right)$ is equal to 0 , resp. to the image of $\epsilon_{q}$ in $K_{0}\left(\mathbb{Z}_{q}[G], \mathbb{Q}_{q}[G]\right)$, if $q \in S_{\text {ram }}$, resp. $q \in S_{\text {bad }}$.

(iii) If condition (B) is satisfied and $p \in \operatorname{Spec}(\Lambda)$, then $\xi_{p}=0$.

Proof. We first fix any odd prime $q$ which does not belong to $S\left(=S_{\text {ram }} \cup S_{\text {bad }}\right.$ ). Then the theory of Fontaine and Messing [46] implies that $\mathcal{J}_{q}=\mathcal{D}_{q} / F^{0} \mathcal{D}_{q}$. In this case it is also known that there is a natural short exact sequence of perfect 
complexes of $\mathbb{Z}_{q}[G]$-modules (with vertical differentials)

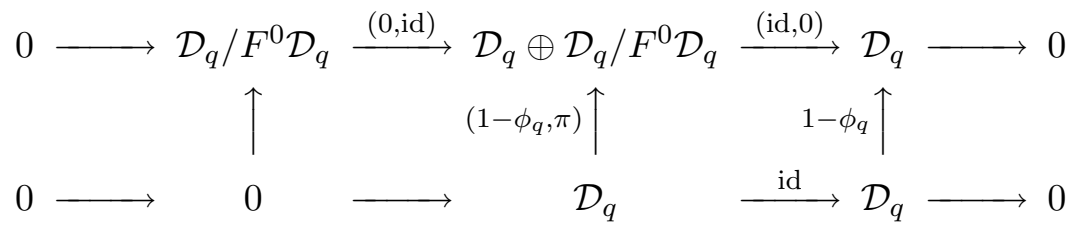

in which the term $\mathcal{D}_{q} / F^{0} \mathcal{D}_{q}$ in the first complex occurs in degree $1, \pi$ is the tautological projection and $\exp _{q}^{\mathrm{BK}}$ maps the cohomology in degree 1 of the second complex bijectively to $H_{f}^{1}\left(\mathbb{Q}_{q}, T_{q}\right)_{\text {BK}}$. Now the differential $1-\phi_{q}$ of the third complex in (56) is injective and so the associated long exact sequence of cohomology gives both an inclusion $\exp _{q}^{\mathrm{BK}}\left(\mathcal{J}_{q}\right) \subset H_{f}^{1}\left(\mathbb{Q}_{q}, T_{q}\right)_{\mathrm{BK}}\left(\right.$ so that $\left.\mathcal{I}_{q}=\exp _{q}^{\mathrm{BK}}\left(\mathcal{J}_{q}\right)\right)$ and an isomorphism $H_{f}^{1}\left(\mathbb{Q}_{q}, T_{q}\right)_{\mathrm{BK}} / \exp _{q}^{\mathrm{BK}}\left(\mathcal{J}_{q}\right) \cong \operatorname{cok}\left(1-\phi_{q} \mid \mathcal{D}_{q}\right)$. To deduce claim (i) from this we therefore need only note that if also $q \nmid|G|$, then the description of $L_{q}(\mathbb{Q}[G] M, s)$ in terms of the action of $1-\phi_{q}$ on $\mathbb{Q}_{q} \otimes_{\mathbb{Z}_{q}} \mathcal{D}_{q}$ implies that $-\operatorname{cl}_{q}\left(\operatorname{cok}\left(1-\phi_{q} \mid \mathcal{D}_{q}\right)\right)$ is equal to the image of $\epsilon_{q}$ in $K_{0}\left(\mathbb{Z}_{q}[G], \mathbb{Q}_{q}[G]\right.$ ) (cf. [23, $\S 4.1$, Rem. 7]).

To prove claim (ii) we fix a prime $q$ in $\operatorname{Spec}(\Lambda)$ with $q \nmid|G|$ and set

$$
C_{q}^{\bullet}:=R \Gamma\left(\mathbb{R}, T_{q}\right) \oplus R \Gamma_{f}\left(\mathbb{Q}_{q}, T_{q}\right) \cong H^{0}\left(\operatorname{Gal}(\mathbb{C} / \mathbb{R}), T_{q}\right)[0] \oplus H^{1}\left(R \Gamma_{f}\left(\mathbb{Q}_{q}, T_{q}\right)\right)[-1] .
$$

Then the definitions of $R \Gamma_{c}\left(\mathbb{Z}_{S}\left[\frac{1}{q}\right], T_{q}\right)$ and $R \Gamma_{f}\left(\mathbb{Q}, T_{q}\right)$ combine to give a natural exact triangle in $D^{\text {perf }}\left(\mathbb{Z}_{q}[G]\right)$ of the form

$$
R \Gamma_{c}\left(\mathbb{Z}_{S}\left[\frac{1}{q}\right], T_{q}\right) \rightarrow R \Gamma_{f}\left(\mathbb{Q}, T_{q}\right) \rightarrow C_{q}^{\bullet} \oplus \bigoplus_{\ell \in S \backslash\{q\}} R \Gamma_{f}\left(\mathbb{Q}_{\ell}, T_{q}\right) \rightarrow .
$$

For each embedding $j: \mathbb{R} \rightarrow \mathbb{C}_{q}$ we write $\iota_{j}$ for the induced embedding of relative $K$-groups $K_{0}(\Lambda[G], \mathbb{R}[G]) \rightarrow K_{0}\left(\mathbb{Z}_{q}[G], \mathbb{C}_{q}[G]\right)$. Then the very definition of $R \Omega(M, \Lambda[G])$ makes it clear that

$$
\iota_{j}(R \Omega(M, \mathbb{Z}[G]))=\left[R \Gamma_{c}\left(\mathbb{Z}_{S}\left[\frac{1}{q}\right], T_{q}\right), \theta_{q}\right] \in K_{0}\left(\mathbb{Z}_{q}[G], \mathbb{C}_{q}[G]\right)
$$

where the morphism $\theta_{q}:\left[\mathbb{C}_{q} \otimes_{\mathbb{Z}_{q}} R \Gamma_{c}\left(\mathbb{Z}_{S}\left[\frac{1}{q}\right], T_{q}\right)\right] \rightarrow \mathbf{1}_{\mathbb{C}_{q}[G]}$ is constructed using (57), the canonical comparison isomorphisms associated to $M$ and the morphism $\lambda_{j}:\left[\mathbb{C}_{q} \otimes_{\mathbb{Z}_{q}} R \Gamma_{f}\left(\mathbb{Q}, T_{q}\right)\right] \rightarrow \mathbf{1}_{\mathbb{C}_{q}[G]}$ that is induced by the displayed exact sequence in the proof of Lemma 12.2.2 and the image under $\mathbb{C}_{q} \otimes_{\mathbb{R}, j}$ - of the Néron-Tate height pairing. On the other hand, the definition of the a.t.e. $\tau^{\prime}$ in $\S 12.3$ implies that

$$
\iota_{j}\left(\left[\epsilon_{\tau^{\prime}}, \lambda_{\tau^{\prime}}\right]\right)=\left[R \Gamma_{f}\left(\mathbb{Q}, T_{q}\right)[1], \lambda_{j}^{-1}\right]=-\left[R \Gamma_{f}\left(\mathbb{Q}, T_{q}\right), \lambda_{j}\right] \in K_{0}\left(\mathbb{Z}_{q}[G], \mathbb{C}_{q}[G]\right) .
$$


Now, as recalled in the proof of Lemma 12.3.1, $T \Omega(M, \Lambda[G])=R \Omega(M, \Lambda[G])+$ $\delta_{\Lambda}\left(\mathcal{L}_{\tau^{\prime}}^{*}\right)$ and therefore $\left[\epsilon_{\tau^{\prime}}, \lambda_{\tau^{\prime}}\right]+R \Omega(M, \Lambda[G])=\chi\left(\tau^{\prime}\right)+T \Omega(M, \Lambda[G])$. Hence, upon combining the above displayed formulas for $\iota_{j}(R \Omega(M, \mathbb{Z}[G]))$ and $\iota_{j}\left(\left[\epsilon_{\tau^{\prime}}, \lambda_{\tau^{\prime}}\right]\right)$, making an explicit comparison of all of the maps involved and taking account of the remark which immediately follows $[23,(24)]$ one finds that

$$
\begin{aligned}
& \iota_{j}\left(\chi\left(\tau^{\prime}\right)+T \Omega(M, \Lambda[G])\right) \\
= & \iota_{j}\left(\left[\epsilon_{\tau^{\prime}}, \lambda_{\tau^{\prime}}\right]\right. \\
=- & R \Omega(M, \Lambda[G])) \\
=-\mathrm{cl}_{q}\left(\mathbb{Z}_{q} \otimes_{\Lambda}\right. & \left.H_{B, \Lambda}^{+}, H_{f}^{1}\left(\mathbb{Q}_{q}, T_{q}\right)_{\mathrm{BK}} ; \exp _{q}^{\mathrm{BK}} \circ \pi_{j}\right) \\
& +\sum_{\ell \in S \cup\{q\}} \iota j\left(\epsilon_{\ell}\right)+\sum_{\ell \in S \backslash\{q\}} \operatorname{cl}_{q}\left(H_{f}^{1}\left(\mathbb{Q}_{\ell}, T_{q}\right)_{\mathrm{BK}}\right) .
\end{aligned}
$$

One also has

$$
\begin{aligned}
& \operatorname{cl}_{q}\left(\mathbb{Z}_{q} \otimes_{\Lambda} H_{B, \Lambda}^{+}, H_{f}^{1}\left(\mathbb{Q}_{q}, T_{q}\right)_{\mathrm{BK}} ; \exp _{q}^{\mathrm{BK}} \circ \pi_{j}\right) \\
=\iota_{j} & \left(\left(H_{B, \Lambda}^{+}, \Lambda \otimes H^{1}\left(\mathcal{E}_{K}, \mathcal{O}_{\mathcal{E}_{K}}\right) ; \pi\right)\right) \\
\quad & \quad \operatorname{cl}_{q}\left(\mathbb{Z}_{q} \otimes H^{1}\left(\mathcal{E}_{K}, \mathcal{O}_{\mathcal{E}_{K}}\right), H_{f}^{1}\left(\mathbb{Q}_{q}, T_{q}\right)_{\mathrm{BK}} ; \exp _{q}^{\mathrm{BK}} \circ \kappa_{q}\right) \\
=\iota_{j} & \left(\left(H_{B, \Lambda}^{+}, \Lambda \otimes H^{1}\left(\mathcal{E}_{K}, \mathcal{O}_{\mathcal{E}_{K}}\right) ; \pi\right)\right) \\
\quad & \quad \operatorname{cl}_{q}\left(\exp _{q}^{\mathrm{BK}}\left(\mathcal{J}_{q}\right) / \mathcal{I}_{q}\right)-\operatorname{cl}_{q}\left(H_{f}^{1}\left(\mathbb{Q}_{q}, T_{q}\right)_{\mathrm{BK}} / \mathcal{I}_{q}\right) .
\end{aligned}
$$

In addition, for each $\ell \in S \backslash\{q\}$ the exact sequence

$$
0 \rightarrow \operatorname{cok}\left(1-\mathrm{Fr}_{\ell}^{-1} \mid T_{q}^{I_{\ell}}\right) \rightarrow H_{f}^{1}\left(\mathbb{Q}_{\ell}, T_{q}\right)_{\mathrm{BK}} \rightarrow H^{0}\left(\mathbb{Q}_{\ell}, H^{1}\left(I_{\ell}, T_{q}\right)_{\text {tor }}\right) \rightarrow 0
$$

of $[21,(1.38)]$ combines with $[23$, Rem. 7$]$ to imply that

$$
\operatorname{cl}_{q}\left(H_{f}^{1}\left(\mathbb{Q}_{\ell}, T_{q}\right)_{\mathrm{BK}}\right)+\iota_{j}\left(\epsilon_{\ell}\right)=\mathrm{cl}_{q}\left(H^{0}\left(\mathbb{Q}_{\ell}, H^{1}\left(I_{\ell}, T_{q}\right)_{\text {tor }}\right)\right) .
$$

Taking account of these formulas, and noting that $H^{0}\left(\mathbb{Q}_{\ell}, H^{1}\left(I_{\ell}, T_{q}\right)_{\text {tor }}\right)$ vanishes for every $\ell \notin S_{\text {bad }}$, it is straightforward to check that (58) implies the explicit formula for $\xi_{q}$ given in claim (ii).

To prove claim (iii) we now assume that condition (B) is satisfied, that $q=p$ and that $p \in \operatorname{Spec}(\Lambda)$ (the latter condition being automatically satisfied if, for example, $\Lambda=\mathbb{Z}\left[\frac{1}{2}\right]$ ). Then Lemmas 12.2 .1 and 12.2 .2 combine to imply that there is an exact triangle of perfect complexes of $\mathbb{Z}_{p}[G]$-modules of the form (57) (here we are also using the fact that $p$ is odd). In addition, our assumptions (B)(ii) and (B)(iv) on $p$ together imply that $p \notin S$ and hence both that there is a short exact sequence of perfect complexes of the form (56) with $q=p$ and that $\kappa_{p}\left(\mathbb{Z}_{p} \otimes H^{1}\left(\mathcal{E}_{K}, \mathcal{O}_{\mathcal{E}_{K}}\right)\right)=\mathcal{D}_{p} / F^{0} \mathcal{D}_{p}$. By using these facts an argument similar to 
the above shows that if $j$ is any embedding $\mathbb{R} \rightarrow \mathbb{C}_{p}$, then the correct analogue of (58) is the formula

$$
\begin{aligned}
& \iota_{j}\left(\chi\left(\tau^{\prime}\right)+T \Omega(M, \Lambda[G])\right) \\
= & -\left(\mathbb{Z}_{p} \otimes_{\Lambda} H_{B, \Lambda}^{+}, \mathcal{D}_{p} / F^{0} \mathcal{D}_{p} ; \pi_{j}\right)+\sum_{\ell \in S_{\mathrm{ram}}} \iota_{j}\left(\epsilon_{\ell}\right) \\
= & -\iota_{j}\left(\left(H_{B, \Lambda}^{+}, \Lambda \otimes H^{1}\left(\mathcal{E}_{K}, \mathcal{O}_{\mathcal{E}_{K}}\right) ; \pi\right)\right)+\sum_{\ell \in S_{\mathrm{ram}}} \iota_{j}\left(\epsilon_{\ell}\right),
\end{aligned}
$$

or equivalently, $\iota_{j}(\xi)=0$. It is therefore clear that $\xi_{p}=0$, as required.

Remark 12.4.2. Finite support cohomology

The second complex in the sequence (56) is naturally quasi-isomorphic to the complex $F^{0} \mathcal{D}_{q} \stackrel{1-\phi_{q}^{0}}{\longrightarrow} \mathcal{D}_{q}$ in which the first term occurs in degree 0 . In addition, it is acyclic in degree 0 and so $\exp _{q}^{\mathrm{BK}}$ induces a quasi-isomorphism from it to the complex $H_{f}^{1}\left(\mathbb{Q}_{q}, T_{q}\right)_{\mathrm{BK}}[-1]$. This shows that for any odd prime $q$ which does not belong to $S$ it is possible to define the complex $R \Gamma_{f}\left(\mathbb{Q}_{q}, T_{q}\right)$ introduced at the beginning of $\S 12.2$ in exactly the same way as we defined $R \Gamma_{f}\left(\mathbb{Q}_{p}, T_{p}\right)$ under condition (B).

\subsection{Completion of the proof.}

Lemma 12.5.1. Under the conditions of Proposition 4.3.1 the $\Lambda[G]$-module $\Lambda \otimes$ $H^{1}\left(\mathcal{E}_{K}, \mathcal{O}_{\mathcal{E}_{K}}\right)$ is projective of rank one and in $K_{0}(\Lambda[G], \mathbb{R}[G])$ one has

$$
\left(H_{B, \Lambda}^{+}, \Lambda \otimes H^{1}\left(\mathcal{E}_{K}, \mathcal{O}_{\mathcal{E}_{K}}\right) ; \pi\right)=\delta_{\Lambda}\left(\left(\Omega(E)^{\psi(1)} \tau^{*}(\psi)^{-1}\right)_{\psi}\right) .
$$

Proof. Under either condition (A) or (B) of Proposition 4.3.1 the highest common factor of $\operatorname{cond}(E)$ and $\operatorname{disc}(K)$ is invertible in $\Lambda$ and so $\mathcal{E}_{K, \Lambda}$ is isomorphic to $\mathcal{O}_{K, \Lambda} \times_{\mathbb{Z}} \mathcal{E}$ where $\mathcal{E}$ is the Néron model of $E$ over $\mathbb{Z}$ (cf. [2, Cor. 1.4]). Hence $\Lambda \otimes H^{1}\left(\mathcal{E}_{K}, \mathcal{O}_{\mathcal{E}_{K}}\right)$ is isomorphic as a $\Lambda[G]$-module to $\mathcal{O}_{K, \Lambda} \otimes H^{1}\left(\mathcal{E}, \mathcal{O}_{\mathcal{E}}\right)$. Thus, since either $|G|$ is invertible in $\Lambda$ (under condition $(\mathrm{A})$ ) or $K / \mathbb{Q}$ is tamely ramified (under condition (B)), Noether's Theorem implies that the $\Lambda[G]$-module $\mathcal{O}_{K, \Lambda}$, and hence also $\mathcal{O}_{K, \Lambda} \otimes H^{1}\left(\mathcal{E}, \mathcal{O}_{\mathcal{E}}\right) \cong \Lambda \otimes H^{1}\left(\mathcal{E}_{K}, \mathcal{O}_{\mathcal{E}_{K}}\right)$, is projective of rank one. We fix a free (rank one) $\mathcal{O}_{\Lambda}[G]$-submodule $\mathcal{F}$ of $\mathcal{O}_{K, \Lambda}$.

Now, by Serre duality, $H^{1}\left(\mathcal{E}, \mathcal{O}_{\mathcal{E}}\right)$ is canonically isomorphic to the linear dual $H^{0}\left(\mathcal{E}, \Omega_{\mathcal{E}}^{1}\right)^{*}:=\operatorname{Hom}_{\mathbb{Z}}\left(H^{0}\left(\mathcal{E}, \Omega_{\mathcal{E}}^{1}\right), \mathbb{Z}\right)$. Further, by choosing $\mathbb{Z}$-bases $\theta$ and $\phi$ of the 
groups $H^{0}\left(\operatorname{Gal}(\mathbb{C} / \mathbb{R}), \operatorname{Hom}_{\mathbb{Z}}\left(H_{1}(E(\mathbb{C}), \mathbb{Z}), 2 \pi i \mathbb{Z}\right)\right)$ and $H^{0}\left(\mathcal{E}, \Omega_{\mathcal{E}}^{1}\right)^{*}$ and a $\Lambda[G]$ basis $\alpha$ of $\mathcal{F}$, and then computing the matrix of $\pi$ with respect to the $\Lambda[G]$-bases $1 \otimes \theta$ and $\alpha \otimes \phi$ of $H_{B, \Lambda}^{+}$and $\mathcal{F} \otimes H^{0}\left(\mathcal{E}, \Omega_{\mathcal{E}}^{1}\right)^{*}$ one finds that

$$
\begin{aligned}
& \left(H_{B, \Lambda}^{+}, \Lambda \otimes H^{1}\left(\mathcal{E}_{K}, \mathcal{O}_{\mathcal{E}_{K}}\right) ; \pi\right) \\
= & \left(H_{B, \Lambda}^{+}, \mathcal{F} \otimes H^{0}\left(\mathcal{E}, \Omega_{\mathcal{E}}^{1}\right)^{*} ; \pi\right)+\left(\mathcal{F} ; \mathcal{O}_{K, \Lambda} ; \mathrm{id}\right) \\
= & \delta_{\Lambda}\left(\left(\Omega(E)^{\psi(1)}\right)_{\psi}\right)-(\mathcal{F}, \Lambda[G] ; \hat{\pi})+\left(\mathcal{F} ; \mathcal{O}_{K, \Lambda} ; \mathrm{id}\right) \\
= & \delta_{\Lambda}\left(\left(\Omega(E)^{\psi(1)}\right)_{\psi}\right)-\left(\mathcal{O}_{K, \Lambda}, \Lambda[G] ; \hat{\pi}\right)
\end{aligned}
$$

where $\hat{\pi}$ is the canonical isomorphism $\mathbb{R} \otimes_{\mathbb{Q}} K \cong \prod_{\operatorname{Hom}(K, \mathbb{C})} \mathbb{R} \cong \mathbb{R}[G]$ (which exists since $K$ is totally real). It remains to compute the element $\left(\mathcal{O}_{K, \Lambda}, \Lambda[G] ; \hat{\pi}\right)$ and to do this we use results of Bley and the present author from [8]. Indeed, if condition (B) is satisfied, then $K / \mathbb{Q}$ is tamely ramified and the results of $[8$, Cor. 7.7 and Rem. 3.5] combine to imply that $\left(\mathcal{O}_{K}, \mathbb{Z}[G] ; \hat{\pi}\right)=\delta\left(\left(\tau^{*}(\psi)\right)_{\psi}\right)$. On the other hand, if condition (A) is satisfied, then $|G|$ is invertible in $\Lambda$ and the results of [8, Cor. 7.6, Prop. 7.1 and Rem. 3.5] can be combined to deduce that $\left(\mathcal{O}_{K, \Lambda}, \Lambda[G] ; \hat{\pi}\right)=\delta_{\Lambda}\left(\left(\tau^{*}(\psi)\right)_{\psi}\right)$. The claimed equality is now clear.

To proceed we write $\Sigma$ for the set of all primes in $\operatorname{Spec}(\Lambda)$ which do not divide $|G|$. We then define $\Lambda[G]$-modules by setting

$$
c_{E, \Lambda}^{0}(K):=\bigoplus_{q \in \Sigma \cap S}\left[B_{q} \oplus \exp _{q}^{\mathrm{BK}}\left(\mathcal{J}_{q}\right) / \mathcal{I}_{q}\right]
$$

and

$$
\begin{aligned}
c_{E, \Lambda}^{1}(K):= & \bigoplus_{q \in \Sigma \cap S}\left[A_{q} \oplus H_{f}^{1}\left(\mathbb{Q}_{q}, T_{q}\right)_{\mathrm{BK}} / \mathcal{I}_{q}\right] \\
& \oplus \bigoplus_{\substack{q \in \Sigma \\
\ell \in S_{\mathrm{bad} \backslash\{q\}}}} H^{0}\left(\mathbb{Q}_{\ell}, H^{1}\left(I_{\ell}, T_{q}\right)_{\mathrm{tor}}\right) \oplus \bigoplus_{\substack{q \\
\ell \in S_{\mathrm{ram} \backslash\{q\}}}} \operatorname{cok}\left(1-\operatorname{Fr}_{\ell}^{-1} \mid T_{q}^{I_{\ell}}\right) .
\end{aligned}
$$

Here we use the notation of Lemma 12.4.1 and in the last direct sum $q$ runs over the finite set of primes in $\Sigma$ for which there exists a prime $\ell$ in $S_{\text {ram }} \backslash\{q\}$ such that the image $\operatorname{cl}_{q}\left(\operatorname{cok}\left(1-\operatorname{Fr}_{\ell}^{-1} \mid T_{q}^{I_{\ell}}\right)\right)$ of $-\epsilon_{\ell}$ under the natural projection $K(\mathbb{Z}[G], \mathbb{Q}[G]) \rightarrow K_{0}\left(\mathbb{Z}_{q}[G], \mathbb{Q}_{q}[G]\right)$ is non-zero. Note that, in terms of the notation used in Proposition 4.3.1, the last condition is equivalent to asserting that the image of $P_{\ell}(E, K)$ in $\zeta\left(\mathbb{Q}_{q}[G]\right)^{\times} \cong K_{1}\left(\mathbb{Q}_{q}[G]\right)$ does not belong to the image of the natural map $K_{1}\left(\mathbb{Z}_{q}[G]\right) \rightarrow K_{1}\left(\mathbb{Q}_{q}[G]\right)$. Now whilst $c_{E, \Lambda}^{0}(K)$ is clearly finite the module $c_{E, \Lambda}^{1}(K)$ is also finite because for each prime $\ell$ in $S_{\text {bad }}$ the direct 
sum $\bigoplus_{q \in \Sigma} H^{0}\left(\mathbb{Q}_{\ell}, H^{1}\left(I_{\ell}, T_{q}\right)_{\text {tor }}\right)$ is finite (see $\left.\S 12.1\right)$. Hence we can define $\tau_{E, \Lambda}$ to be the a.t.e. of $\Lambda[G]$-modules which differs from $\tau^{\prime}$ only in the respect that $H_{\tau_{E, \Lambda}}^{0}:=H_{\tau^{\prime}}^{0} \oplus c_{E, \Lambda}^{0}(K), H_{\tau_{E, \Lambda}}^{1}:=H_{\tau^{\prime}}^{1} \oplus c_{E, \Lambda}^{1}(K)$ and

$$
\begin{aligned}
\left(\mathcal{L}_{\tau_{E, \Lambda}}^{*}\right)_{\psi}:=\left(\mathcal{L}_{\tau^{\prime}}^{*}\right)_{\psi} \cdot \Omega(E)^{-\psi(1)} \tau^{*}(\psi) \prod_{\ell \in S_{\mathrm{ram}}} L_{\ell}(\mathbb{Q}[G] & M, 0)_{\psi} \\
& =\Omega(E)^{-\psi(1)} \tau^{*}(\psi) L_{S_{\mathrm{ram}}}^{*}(E, \bar{\psi}, 1)
\end{aligned}
$$

for each $\psi$ in $\operatorname{Irr}(G)$. A straightforward computation shows that, with this definition of $\tau_{E, \Lambda}$, the results of Lemmas 12.4 .1 and 12.5 .1 combine to imply

$$
\chi\left(\tau_{E, \Lambda}\right)=-T \Omega\left(h^{1}\left(E_{/ K}\right)(1), \Lambda[G]\right)
$$

and hence that all of the claims made in Proposition 4.3.1 are valid.

\section{REFERENCES}

[1] A. Agboola, D. Burns, Twisted forms and relative $K$-theory, Proc. London Math. Soc. 92 (2006) 1-28.

[2] M. Artin, Néron models, in 'Arithmetic Geometry', G. Cornell, J. Silverman (eds.), 213-230, Springer Verlag, 1986.

[3] M. F. Atiyah, C. T. C. Wall, Cohomology of Groups, in 'Algebraic Number Theory', J. W. S. Cassels, A. Fröhlich (eds.), 94-115, Academic Press, London, 1967.

[4] M. Auslander, D. A. Buchsbaum, Homological dimension in local rings, Trans. Amer. Math. Soc. 85 (1957) 390-405.

[5] A-M. Bergé, Arithmétique d'une extension à groupe d'inertie cyclique, Ann. Inst. Fourier 28 (1978) 17-44.

[6] W. Bley, On the equivariant Tamagawa number conjecture for abelian extensions of a quadratic imaginary field, Documenta Math. 11 (2006) 73-118.

[7] W. Bley, Numerical evidence for the equivariant Birch and Swinnerton-Dyer conjecture, preprint, 2009.

[8] W. Bley, D. Burns, Equivariant epsilon constants, discriminants and étale cohomology, Proc. London Math. Soc. 87 (2003) 545-590.

[9] S. Bloch, K. Kato, $L$-functions and Tamagawa numbers of motives, In: 'The Grothendieck Festschrift' vol. 1, Progress in Math. 86, Birkhäuser, Boston, (1990) 333-400.

[10] M. Breuning, Equivariant local epsilon constants and étale cohomology, J. London Math. Soc. (2) 70 (2004), 289-306.

[11] M. Breuning, Determinant functors on triangulated categories, preprint 2006. arXiv:math.CT/0610435

[12] M. Breuning, D. Burns, Additivity of Euler characteristics in relative algebraic $K$-groups, Homology, Homotopy and Applications 7 No. 3 (2005) 11-36. 
[13] M. Breuning, D. Burns, Leading terms of Artin $L$-functions at $s=0$ and $s=1$, Compositio Math. 143 (2007) 1427-1464.

[14] M. Breuning, D. Burns, On equivariant Dedekind Zeta functions, manuscript in preparation.

[15] J. I. Burgos Gil, The regulators of Beilinson and Borel, CRM Monograph Series 15, Amer. Math. Soc., 2002.

[16] D. Burns, Equivariant Tamagawa Numbers and Galois module theory, Compositio Math. 129 (2001) 203-237.

[17] D. Burns, On the values of equivariant Zeta functions of curves over finite fields, Documenta Math. 9 (2004) 357-399.

[18] D. Burns, Perfecting the nearly perfect, Pure Appl. Math. Q. 4 (2008) 1041-1058 (JeanPierre Serre Special Issue, Part I).

[19] D. Burns, Congruences between derivatives of abelian $L$-functions at $s=0$, Inventiones Math. 169 (2007) 451-499.

[20] D. Burns, On refined Stark conjectures in the non-abelian case, Math. Res. Letters 15 (2008) 841-856.

[21] D. Burns, M. Flach, Motivic L-functions and Galois module structures, Math. Ann. 305 (1996) 65-102.

[22] D. Burns, M. Flach, On Galois structure invariants associated to Tate motives, Amer. J. Math. 120 (1998) 1343-1397.

[23] D. Burns, M. Flach, Tamagawa numbers for motives with (non-commutative) coefficients, Documenta Math. 6 (2001) 501-570.

[24] D. Burns, M. Flach, Tamagawa numbers for motives with (non-commutative) coefficients II, Amer. J. Math. 125 (2003) 475-512.

[25] D. Burns, M. Flach, On the equivariant Tamagawa number conjecture for Tate motives, Part II, Documenta Math., Extra volume: John H. Coates' Sixtieth Birthday (2006) 133163.

[26] D. Burns, C. Greither, Equivariant Weierstrass Preparation and values of $L$-functions at negative integers, Documenta Math., Extra volume: Kazuya Kato's Fiftieth Birthday (2003) 157-185.

[27] D. Burns, H. Johnston, A non-abelian Stickelberger Theorem, preprint, 2009.

[28] D. Burns, O. Venjakob, On descent theory and main conjectures in non-commutative Iwasawa theory, to appear in J. Inst. Math. Jussieu.

[29] T. Chinburg, On the Galois structure of algebraic integers and $S$-units, Inventiones Math. 74 (1983) 321-349.

[30] T. Chinburg, Stark's Conjecture for $L$-Functions with First-Order Zeroes at $s=0$, Adv. Math. 48 (1983) 82-113.

[31] T. Chinburg, Exact sequences and Galois module structure, Annals of Math. 121 (1985) 351-376.

[32] T. Chinburg, The analytic theory of multiplicative Galois structure, Memoirs of the Amer. Math. Soc., 77 (1989).

[33] T. Chinburg, M. Kolster, G. Pappas, V. P. Snaith, Galois structure of $K$-groups of rings of integers, K-theory 14 (1998), 319-369. 
[34] T. Chinburg, M. Kolster, G. Pappas, V. P. Snaith, Quaternionic exercises in K-theory Galois module structure, Proceedings of the Great Lakes $K$-theory conference, Fields Inst. Commun. Series 16 Amer. Math. Soc., 1997, 1-29.

[35] J. Coates, T. Fukaya, K. Kato, R. Sujatha, O. Venjakob, The $G L_{2}$ main conjecture for elliptic curves without complex multiplication, Publ. IHES 101 (2005) 163-208.

[36] J. H. Coates, W. Sinnott, An analogue of Stickelberger's theorem for the higher $K$-groups, Inventiones Math. 24 (1974) 149-161.

[37] C. W. Curtis, I. Reiner, Methods of Representation Theory, Vols. I and II, John Wiley and Sons, New York, 1987.

[38] P. Deligne, Valeurs de fonctions $L$ et périods d'intégrales, Proc. Sym. Pure Math. 33 (2), (1979) 313-346.

[39] P. Deligne, Le déterminant de la cohomologie, in: 'Current Trends in Arithmetical Algebraic Geometry', Contemp. Math. 67 313-346, Amer. Math. Soc., 1987.

[40] T. Dokchister, V. Dokchister, Some computations in non-commutative Iwasawa theory, Proc. London Math. Soc. (3) 94 (2006) 211-272.

[41] W. G. Dwyer, E. M. Friedlander, Algebraic and étale $K$-theory, Trans. Amer. Math. Soc. 292 (1985), 247-280.

[42] G. G. Elder, A cohomological characterisation of permutation lattices, preprint 2006.

[43] J. Fearnley, H. Kisilevsky, Critical values of derivatives of twisted elliptic $L$-functions, to appear in Experimental Math.

[44] M. Flach, Euler characteristics in relative $K$-groups, Bull. London Math. Soc. 32 (2000) 272-284.

[45] M. Flach, The equivariant Tamagawa number conjecture - A survey, in: 'Stark's Conjecture: recent progress and new directions' (eds. D. Burns, C. Popescu, J. Sands and D. Solomon), Contemp. Math. 358 79-126, Amer. Math. Soc., 2004.

[46] J-M. Fontaine, W. Messing, $p$-adic periods and $p$-adic étale cohomology, in: 'Current trends in Arithmetic Algebraic Geometry', Contemp. Math. 67 179-207, Amer. Math. Soc., 1987.

[47] J.-M. Fontaine, B. Perrin-Riou, Autour des conjectures de Bloch et Kato: cohomologie galoisienne et valeurs de fonctions L, In: Motives (Seattle) Proc. Symp. Pure Math. 55, I, (1994) 599-706.

[48] A. Fröhlich, On the absolute Galois group of abelian fields, J. London Math. Soc. 29 (1954) 211-217; 30 (1955), 72-80.

[49] A. Fröhlich, Galois Module Structure of Algebraic Integers, Ergebnisse Math. 1, Springer Verlag, New York, 1983.

[50] A. Fröhlich, Units in real abelian fields, J. Reine Angew. Math. 429 (1992) 191-217.

[51] T. Fukaya, K. Kato, A formulation of conjectures on $p$-adic zeta functions in noncommutative Iwasawa theory, Proc. St. Petersburg Math. Soc. XII (2006) 1-86.

[52] C. Greither, Some cases of Brumer's conjecture for abelian CM extensions of totally real fields, Math. Zeit. 233 (2000) 515-534.

[53] C. Greither, Arithmetic annihilators and Stark-type conjectures, in: 'Stark's Conjecture: recent progress and new directions' (eds. D. Burns, C. Popescu, J. Sands and D. Solomon), Contemp. Math. 358 55-78, Amer. Math. Soc., 2004. 
[54] C. Greither, Determining Fitting ideals of minus class groups via the Equivariant Tamagawa Number Conjecture, Compositio Math. 143 (2007) 1399-1426.

[55] B. H. Gross, On the values of abelian $L$-functions at $s=0$, J. Fac. Sci. Univ. Tokyo Sect. IA Math., 35 (1988) 177-197.

[56] B. H. Gross, Higher regulators and values of Artin $L$-functions, Pure Appl. Math. Q. 1 (2005), no. 1, 1-13.

[57] A. Grothendieck, Groupes de Monodromie en Géométrie Algébrique (SGA 7 I), Lecture Notes in Math. 288, Springer Verlag, 1972.

[58] K. W. Gruenberg, J. Ritter, A. Weiss, A Local Approach to Chinburg's Root Number Conjecture, Proc. London Math. Soc. 79 (1999) 47-80.

[59] R. Hartshorne, Residues and Duality, Lecture Notes in Math. 20, Springer, New York, 1966.

[60] A. Hayward, A class number formula for higher derivatives of abelian $L$-functions, Compositio Math. 140 (2004) 99-129.

[61] A. Huber, G. Kings, Bloch-Kato conjecture and main conjecture of Iwasawa theory for Dirichlet characters, Duke. Math. J. 119 (2003) 393-464.

[62] A. Jones, Dirichlet $L$-functions at $s=1$, Ph.D. Thesis, King's College London, 2007.

[63] B. Kahn, The Quillen-Lichtenbaum Conjecture at the prime 2, preprint.

[64] K. Kato, Iwasawa theory and p-adic Hodge theory, Kodai Math. J. 16 no 1 (1993) 1-31.

[65] K. Kato, Lectures on the approach to Iwasawa theory of Hasse-Weil $L$-functions via $B_{d R}$, Part I, In: Arithmetical Algebraic Geometry (ed. E.Ballico), Lecture Notes in Math. 1553 (1993) 50-163, Springer Verlag, New York, 1993.

[66] K. Kato, $K_{1}$ of some non-commutative completed group rings, $K$-Theory 34 (2005), no. 2, 99-140.

[67] F. Knudsen, D. Mumford, The projectivity of the moduli space of stable curves I: Preliminaries on 'det' and 'Div', Math. Scand. 39 (1976) 19-55.

[68] M. Kurihara, Iwasawa theory and Fitting ideals, J. Reine u. Angew. Math. 561 (2003) 39-86.

[69] M. Levine, The indecomposable $K_{3}$ of a field, Ann Sci. École Norm. Sup. 22 (1989) 255-344.

[70] S. Lichtenbaum, Values of zeta functions, étale cohomology and algebraic $K$-theory, Lecture Notes in Math. 342, 489-501, Springer Verlag, 1973.

[71] S. Lichtenbaum, Letter to J. Tate, 1975.

[72] D. Macias Castillo, On higher order Stickelberger-type theorems, preprint, 2009.

[73] B. Mazur, J. Tate, Refined Conjectures of the Birch and Swinnerton-Dyer Type, Duke Math. J. 54 (1987) 711-750.

[74] J. S. Milne, Arithmetic Duality Theorems, Academic Press, Boston, 1986.

[75] A. S. Merkurjev, A. A. Suslin, The $K_{3}$-group of a field, Izv. Nauk. SSSR 54 (1990) 339-356.

[76] T. Navilarekallu, On the equivariant Tamagawa number conjecture for $A_{4}$-extensions of number fields, J. Number Theory 121 (2006) 67-89.

[77] T. Navilarekallu, Equivariant Birch-Swinnerton-Dyer Conjecture for the Base Change of Elliptic Curves: An Example, Int. Math. Res. Notices (2008).

[78] J. Nekovář, Selmer Complexes, Astérisque 310, S.M.F., Paris, 2006. 
[79] A. Nickel, On the equivariant Tamagawa number conjecture in tame CM extensions, preprint, 2008.

[80] W. Niziol, Cohomology of crystalline representations, Duke Math. J. 71 (1993) 747-791.

[81] A. Parker, Equivariant Tamagawa numbers and non-commutative Fitting invariants, Ph.D. Thesis, King's College London, 2007.

[82] C. Popescu, Base Change for Stark-Type conjectures over $\mathbb{Z}$, J. Reine u. Angew. Math. 542 (2002) 85-111.

[83] J. Ritter, A. Weiss, A Tate sequence for global units, Compositio Math. 102 (1996), no. 2, 147-178.

[84] J. Rognes, C. Weibel, Étale descent for two-primary algebraic $K$-theory of totally imagnary fields, K-Theory 16 (1999) 101-104.

[85] J. Rognes, C. Weibel (with an appendix by M. Kolster), Two-Primary Algebraic K-Theory of Rings of Integers in Number Fields, J. Amer. Math. Soc. 13 (2000) 1-54

[86] K. Rubin, A Stark Conjecture 'over $\mathbb{Z}$ ' for abelian $L$-functions with multiple zeros, Ann. Inst. Fourier 46 (1996) 33-62.

[87] V. P. Snaith, Burns' Equivariant Tamagawa Invariant $T \Omega^{\mathrm{loc}}(N / \mathbb{Q}, 1)$ for some quaternion fields, J. London Math. Soc. 68 (2003) 599-614.

[88] D. Solomon, On Twisted Zeta-Functions at $s=0$ and Partial Zeta-Functions at $s=1$, J. Number Theory 128 (2008) 105-143.

[89] C. Soulé, $K$-théorie des anneaux d'entiers de corps de nombres et cohomologie étale, Inventiones Math. 55 (1979) 251-295.

[90] H. M. Stark, Values of $L$-functions at $s=1 \mathrm{IV}$ : First derivatives at $s=0$. Advances in Math. 35 (1980) 197-235.

[91] H. M. Stark, Derivatives of $L$-series at $s=0$, in 'Automorphic forms, representation theory and arithmetic (Bombay, 1979)', pp. 261-273, Tata Inst. Fund. Res. Studies in Math., 10, Tata Inst. Fund. Res., Bombay, 1981.

[92] R. G. Swan, Algebraic K-theory, Lecture Note in Math. 76, Springer Verlag, 1968.

[93] J. Tate, Relations between $K_{2}$ and Galois Cohomology, Inventiones Math. 36 (1976) 257274.

[94] J. Tate, Les Conjectures de Stark sur les Fonctions $L$ d'Artin en $s=0$ (notes par D. Bernardi et N. Schappacher), Progress in Math., 47, Birkhäuser, Boston, 1984.

[95] J. Tate, Refining Gross's conjecture on the values of abelian $L$-functions, in: 'Stark's Conjecture: recent progress and new directions' (eds. D. Burns, C. Popescu, J. Sands and D. Solomon), Contemp. Math. 358 189-192, Amer. Math. Soc., 2004.

[96] J. L. Verdier, Des Catégories Dérivées des Catégories Abéliennes, Asterisque 239, Soc. Math. France, 1996.

[97] A. V. Yakovlev, Homological definability of $p$-adic representations of a ring with power basis, Izvestia A N SSSR, ser. Math. 34 (1970), 321-342. (Russian)

\section{David Burns}

King's College London 
Dept. of Mathematics

London WC2R 2LS

United Kingdom

E-mail: david.burns@kcl.ac.uk 